\title{
Fetal blood flow velocity waveforms at various conditions of stress : a Doppler ultrasound study in sheep
}

Citation for published version (APA):

Muijsers, G. J. J. M. (1990). Fetal blood flow velocity waveforms at various conditions of stress : a Doppler ultrasound study in sheep. [Doctoral Thesis, Maastricht University]. Maastricht University. https://doi.org/10.26481/dis.19901207gm

Document status and date:

Published: 01/01/1990

DOI:

10.26481/dis.19901207gm

Document Version:

Publisher's PDF, also known as Version of record

\section{Please check the document version of this publication:}

- A submitted manuscript is the version of the article upon submission and before peer-review. There can be important differences between the submitted version and the official published version of record. People interested in the research are advised to contact the author for the final version of the publication, or visit the DOI to the publisher's website.

- The final author version and the galley proof are versions of the publication after peer review.

- The final published version features the final layout of the paper including the volume, issue and page numbers.

Link to publication

\footnotetext{
General rights rights.

- You may freely distribute the URL identifying the publication in the public portal. please follow below link for the End User Agreement:

www.umlib.nl/taverne-license

Take down policy

If you believe that this document breaches copyright please contact us at:

repository@maastrichtuniversity.nl

providing details and we will investigate your claim.
}

Copyright and moral rights for the publications made accessible in the public portal are retained by the authors and/or other copyright owners and it is a condition of accessing publications that users recognise and abide by the legal requirements associated with these

- Users may download and print one copy of any publication from the public portal for the purpose of private study or research.

- You may not further distribute the material or use it for any profit-making activity or commercial gain

If the publication is distributed under the terms of Article $25 \mathrm{fa}$ of the Dutch Copyright Act, indicated by the "Taverne" license above, 
Fetal blood flow velocity waveforms at various conditions of stress

A Doppler ultrasound study in sheep 


\title{
Fetal blood flow velocity waveforms at various conditions of stress
}

\author{
A Doppler ultrasound study in sheep
}

\section{PROEFSCHRIFT}

ter verkrijging van de graad van doctor aan de Rijksuniversiteit Limburg te Maastricht, op gezag van de Rector Magnificus, Prof. Dr. F.I.M. Bonke, volgens het besluit van het College van Dekanen, in het openbaar te verdedigen

op vrijdag, 7 december 1990 om 16.00 uur

$$
\text { door }
$$

Guido Joseph Jean Marie Muijsers

geboren op 18 januari 1960 te Linne 
Promotor:

Co-promotor:

Beoordelingscommissie: Prof. Dr R.S. Reneman (voorzitter)

Prof. Dr C.E. Blanco

Prof. Dr med. W. Künzel,

Justus-Liebig Universität, Giessen, Germany

Prof. Dr A.M. Rudolph,

University of California, San Francisco, USA

Prof. Dr H.J.J. Wellens

CIP-GEGEVENS KONINKLIJKE BIBLIOTHEEK, DEN HAAG

Muijsers, Guido Joseph Jean Marie

Fetal blood flow velocity waveforms at various conditions

of stress : a Doppler ultrasound study in sheep / Guido

Joseph Jean Marie Muijsers. - Maastricht : Datawyse. - Ill.

Proefschrift Maastricht. - Met lit. opg. - Met

samenvatting in het Nederlands.

ISBN 90-5291-038-3

SISO 605.5 UDC 612.64+618.2(043.3) NUGI 743

Trefw.: foetus ; fysiologie / Doppler sonografie.

Produktie en layout: Datawyse Maastricht, Ruud Leliveld Illustratie omslag: Joost Janssen

Druk: Krips Repro Meppel 
Aan mijn ouders

Voor Thea en Hella 


\section{VOORWOORD}

Het in dit proefschrift beschreven onderzoek werd uitgevoerd binnen de afdeling Obstetrie en Gynaecologie van de Rijksuniversiteit Limburg te Maastricht.

Velen hebben bijgedragen aan het tot stand komen van het proefschrift. Met name wil ik mijn dank betuigen aan Dr T.H.M. Hasaart, begeleider en co-promotor. Beste Tom, niet alleen op wetenschappelijk terrein, maar ook op andere gebieden was en ben je een voortreffelijk begeleider.

Prof. Dr J. de Haan, promotor en hoofd van de afdeling. Uw fysiologische benadering van verloskundige problematiek waren voor mij reden naar Maastricht te komen.

I would like to thank Prof. Dr R.S. Reneman, Prof. Dr C.E. Blanco, Prof. Dr W. Kuinzel, Prof. Dr A.M. Rudolph and Prof. Dr H.J.J. Wellens for the attention they paid to my manuscript.

M. Bost, biotechnicus. Beste May, zonder jouw hulpvaardigheid en flexibiliteit was dit boekje nog niet klaar geweest. Jou ben ik bijzondere dank verschuldigd. Hetzelfde geldt voor Dr. C.J. Ruissen. Beste Kees, jouw hulp, begeleiding en kennis bij problemen van allerlei aard waren onmisbaar.

Dr L.L.H. Peeters, beste Louis, wellicht onbewust heb je in de begintijd van het onderzoek een wetenschappelijke basis bij mij gelegd waarvan ik nog lang hoop te profiteren.

Carla Verkeste, Hans van Huisseling en Focco Boekkooi, mede-promovendi. Jullie vriendschap heeft de onderzoeksjaren tot een aangename periode gemaakt.

Frans Slangen, Joyce Suyk en Peter Franssen. Jullie hulp voor, tijdens en na de dier-experimenten heb ik zeer gewaardeerd.

Dr Hubert Schouten wil ik bedanken voor zijn belangeloze bereidheid tot het bespreken van problemen van statistische aard.

Harrie Wetzelaer en Carin Hendrikx waren de onmisbare deskundigen op het gebied van computers en programmatuur.

Dr Arnold Hoeks en Frans Smeets van het laboratorium Ultrageluid waren bij diverse vragen en problemen behulpzaam.

Jan Geilen en medewerkers ondersteunden de electrotechnische zijde van de experimenten. 
Holger Käsemann en Waltje Jager spanden zich ondermeer in voor de betrouwbaarheidsstudie.

Jan Klerkx doceerde op voortreffelijke manier schrijfvaardigheid en leesvaardigheid Engels.

De stafleden, collega-assistenten en secretaresses van de afdeling Obstetrie en Gynaecologie wil ik bedanken voor hun belangstelling voor en hulp bij de voortgang van mijn onderzoek.

Tenslotte wil ik mijn dank betuigen aan diegenen die niet met name genoemd zijn maar toch zeer behulpzaam waren.

Ik ben $U$ allen zeer erkentelijk. 


\section{CONTENTS}

$\begin{array}{ll}\text { CHAPTER } 1 \text { General introduction } & 11\end{array}$

1.1 INTRODUCTION 11

1.1.1 The significance of fetal hypoxic stress 11

$\begin{array}{ll}\text { 1.1.2 Identification of fetal hypoxemia } & 12\end{array}$

1.1.3 Blood flow velocimetry: a method of fetal surveillance? 13

$\begin{array}{ll}\text { 1.1.4 Fetal heart rate auscultation } & 13\end{array}$

1.1.5 Interpretation of fetal heart rate registrations 14

$\begin{array}{ll}\text { 1.1.6 The biophysical profile score } & 15\end{array}$

1.2. FETAL BLOOD FLOW VELOCIMETRY 16

1.2.1 The Doppler principle 16

$\begin{array}{ll}1.2 .2 & \text { The Doppler shift spectrum } \\ 17\end{array}$

$\begin{array}{ll}1.2 .3 & \text { Doppler blood flow velocity waveform indices } \\ 1.2 .4\end{array}$

$\begin{array}{lll}1.2 .4 & \text { Review of the literature } & 18\end{array}$

1.2.5 Questions raised by the literature 25

$\begin{array}{ll}\text { 1.2.6 Placental vascular anatomy and pathology } 25 & 25\end{array}$

$\begin{array}{ll}\text { 1.2.7 Doppler blood flow velocimetry and hemodynamics } & 27\end{array}$

$\begin{array}{ll}1.2 .8 & \text { Regulation of the fetal circulation } \\ 1.3 & 28\end{array}$

1.3 OBJECTIVES OF THE STUDY 31

CHAPTER 2 Methodology and reliability assessment of Doppler blood flow velocity waveform measurements in fetal sheep

CHAPTER 3 The response of the umbilical and femoral artery Pulsatility Indices in fetal sheep to progressively reduced uteroplacental blood flow

CHAPTER 4 The effect of maternal hypoxemia on the umbilical and femoral artery blood flow velocity waveforms and the relationship with mean arterial pressure in fetal sheep 
CHAPTER 5 The response of the umbilical artery Pulsatility Index in fetal sheep to acute and prolonged hypoxemia and acidemia induced by embolization of the uteroplacental microcirculation

CHAPTER 6 The effect of selective umbilical embolization on the common umbilical artery Pulsatility Index and umbilical vascular resistance in fetal sheep

CHAPTER 7 Regional umbilical embolization in sheep results in discordant blood flow velocity waveforms at the proximal and distal segments of the fetoplacental circulation

CHAPTER 8 The effects of hypertensive drugs on parameters derived from blood flow velocity waveforms in the systemic circulation of fetal sheep

CHAPTER 9 General discussion and conclusions

References

Summary

Samenvatting

Curriculum vitae 


\section{CHAPTER 1}

\section{General introduction}

\section{$1.1 \quad$ INTRODUCTION}

Maternal, fetal and postnatal health should be ensured and optimized by obstetric care. The main concern of the obstetrician therefore is to monitor mother and fetus during pregnancy, labour and puerperium and to reach favorable outcome for both, which in practice often implies a pursuit of fetal maturity and spontaneous delivery.

Pregnancy and puerperium may be affected by a wide range of complications, which can develop preconceptionally, during the embryonic and fetal stages, during labor and in the neonatal period. Although each of these stages is characterized by its own, specific problems, a major concern during the postembryonic stages is to protect the fetus and neonate from hypoxia.

\subsubsection{The significance of fetal hypoxic stress}

It has been shown in monkeys that prolonged hypoxia for periods of 3-5 hours results in brain damage while the severity of this damage depends on the degree and duration of the hypoxic insult (Myers et al., 1969; Myers, 1972). Permanent cerebral lesions were observed in the monkey when the fetus was exposed to oxygen saturation levels of less than $30 \%$ and $\mathrm{pH}$ levels of less than 7.0 (Myers et al., 1969), although fetuses exposed to even more severe degrees of hypoxia did not exhibit cerebral lesions (Myers, 1972). Correponding data are available from the human fetus. Logistic stepwise regression analysis showed that intrapartum fetal hypoxia, expressed by an umbilical artery buffer base of less than $30 \mathrm{mM}$ contributed to $12 \%$ of the cases with mild to moderate encephalopathy and $22 \%$ of the cases with severe encephalopathy in a group of 91 children with newborn encephalopathy (Low et al., 1985). In addition, 
whereas the majority of cases of newborn encephalopathy in immature and early premature infants are caused by neonatal respiratory complications and infections, the majority of such cases in late premature and mature infants appear to develop on the basis of intrauterine hypoxia (Volpe, 1977). These observations are supported by the animal experimental evidence that acute severe hypoxia in normally grown fetal lambs results in cerebral damage, whereas chronic mild hypoxia in growth-retarded fetal lambs does not have these sequelae (Clapp et al., 1981). Furthermore, in normally-developed term infants with Apgar scores of 3 or less at 15-20 minutes after delivery, unidentified intrauterine hypoxia and fetal cerebral injury prior to labor probably account for a mortality rate of $53-59 \%$ and a subsequent cerebral palsy rate of $36-57 \%$ (Nelson \& Ellenberg, 1981). The results of a multivariate analysis of risk factors which precede cerebral palsy, suggest a relatively small role for events during labor and a larger role for hypoxia prior to labor (Nelson \& Ellenberg, 1986). In conclusion, a major objective of obstetric care should be to correctly monitor fetal oxygenation in high-risk pregancies and to establish appropriate management prior to the occurrence of intrauterine hypoxic insults. For lack of causal or preventive therapy by now, the latter frequently implies accurate timing of elective delivery.

\subsubsection{Identification of fetal hypoxemia}

Direct determination of fetal oxygenation requires access to the fetal circulation. In the antenatal period, this is possible by cordocentesis, which has recently been introduced in obstetrics (Rodeck \& Campbell, 1978; Daffos et al., 1983; Nicolaides et al., 1986) and which consists of blood sampling from the umbillical vein. Preferably however, methods to assess fetal welfare should be noninvasive, harmless and little bothering for mother and fetus, useful as a screening method, easily and repetitively to apply by every physician involved in obstetrics, and inexpensive. Obviously, cordocentesis does not comply with these conditions.

Current noninvasive fetal surveillance comprises three widely used biophysical methods, each of them not evaluated for their cost and effectiveness. These methods are: fetal heart rate auscultation using a monaural stethoscope (Lejumeau de Kergaradec, 1821), registration of the fetal heart rate pattern using Doppler ultrasound (Callagan et al., 1964), and biophysical profile scoring using two-dimensional and Doppler ultrasound (Manning et al., 1980). A fourth 
method, Doppler blood flow velocimetry, introduced by FitzGerald and Drumm in 1977 , is not yet subject to wide-spread routine clinical use, but is still gaining interest.

\subsubsection{Blood flow velocimetry: a method of fetal surveillance?}

This study was undertaken to uncover some of the basic characteristics of fetal Doppler blood flow velocimetry and to get insight in the potential clinical usefulness of this diagnostic technique as a method of fetal surveillance. The study was performed in chronically-instrumented and anesthesized fetal sheep, for decades the mostly used model for the study of fetal physiology. After a short description of the limitations of the three routine biophysical methods of fetal surveillance, some basic information will be given on fetal Doppler blood flow velocimetry. In a concise literature review, the pioneering studies on fetal Doppler blood flow velocimetry and those which refer to fetal surveillance and management will be discussed. Introductory remarks concerning the circulatory anatomy in fetal sheep, as far as relevant to the study of the fetal circulation using Doppler ultrasound, will follow. Next, the role of Doppler ultrasound in the study of hemodynamics will be summarized, followed by a short overview of the regulation mechanisms of the fetal circulation at conditions of stress. Finally, a more detailed description will be given of the objectives of this study.

\subsubsection{Fetal heart rate auscultation}

In 1821, Lejumeau de Kergaradec described that it was possible to diagnose single and plural pregnancy, to determine the state of fetal health and the fetal position using Laennec's stethoscope (Goodlin, 1979). In continental Europe however, fetal heart rate auscultation as a method of fetal surveillance, was not adopted by physicians earlier than the end of the nineteenth century, when Winckel (1893) proposed definite criteria for fetal distress, being a fetal heart rate under 120 or over 160 beats per minute.

From the first controlled study on intrapartum fetal heart heart rate auscultation as a diagnostic test for fetal compromise, it was concluded that the reknown signs of fetal distress were of little predictive value concerning fetal outcome (Walker, 1959). Walker demonstrated in a relatively large series that operative interference in cases of suspected fetal distress (according to criteria available 
at that time) did not improve the neonate's condition and suggested that the clinical criteria of intrapartum fetal distress were probably inadequate (Goodlin, 1979).

In antenatal care, fetal heart rate auscultation should be regarded as a screening test for fetal integrity rather as a diagnostic test for fetal compromise. As far as known, no trials have been undertaken to evaluate the value of fetal heart rate auscultation in antenatal care and to compare this screening method with anamnestic maternal information on fetal movements. The method of maternal inquiry potentially encloses a reduction of perinatal mortality without known contraindications and exceeding cost (Thacker \& Berkelman, 1986; Grant et al., 1989a). Although the proposal for such a trial might sound rather absurd in a time in which availability of high-technology medical systems seems unlimited, the question of effectiveness of the procedure of auscultation itself, remains.

\subsubsection{Interpretation of fetal heart rate registrations}

In modern obstetrics, characteristics of the continuously recorded fetal heart rate are used for diagnosis and management of presumed fetal compromise (Wheble et al., 1989). In the antenatal period and during labour, when the fetal membranes are intact, fetal heart rate is most frequently recorded using a Doppler ultrasound transducer (Callagan et al., 1964). During labour, when the membranes are ruptured and cervical dilatation is $1 \mathrm{~cm}$ or more, fetal heart rate is often recorded from the fetal scalp using the spiral electrode according to Hon et al. (1972). After extensive research on the characteristics of the fetal heart rate pattern during labour by, among others, Hon \& Quilligan (1968) and Caldeyro-Barcia et al. (1968). Haverkamp et al. (1976) were the first who performed a randomized controlled trial to examine the efficacy of electronic fetal monitoring in high-risk pregnancies during labor. It was concluded that electronic fetal monitoring resulted in an increase in cesarean section rate and did not improve perinatal morbidity rates. The same conclusion could be drawn from a subsequent series of five trials (Thacker, 1987; Prentice \& Lind, 1987). In an assessment of the quality of seven randomized controlled trials on the efficacy of electronic fetal monitoring available at the time of evaluation, the so-called Dublin trial was considered to be the best on the basis of its excellent design and implementation (Thacker, 1987). This trial demonstrated a statistically significant decrease in the occurrence of neonatal seizures in the electronic fetal monitoring group (MacDonald et al., 1985). The 55\% decrease in neonatal seizures ( 27 in the auscultation group versus 12 in the electronic fetal monitoring group) appeared to be associated with the use of oxytocine in prolonged 
labor. Six of the 39 infants ( 3 in each group) showed neurological sequelae at 1 year of age. There were no differences in the rate of perinatal death, stillbirth, or neonatal death. There were no differences in the rates of low Apgar score, admissions to neonatal intensive care, or in other types of neonatal morbidity. However, the rate of operative deliveries was significantly increased in the electronic fetal monitoring group. Reassessment of the majority of infants included in the Dublin trial at 4 years of age, showed that 22 had cerebral palsy: the original 6 ( 3 in each in group) and 16 (also equally distributed over both study groups) who had not shown clinical signs suggestive of intrapartum asphyxia neonatally (Grant et al., 1989b). It was concluded that electronic fetal monitoring has little, if any, protective effect against cerebral palsy or neurological deficit.

In randomized controlled trials evaluating the efficacy of antenatal fetal heart rate monitoring, no statistical significant differences in fetal distress, low Apgar scores, induction of labour and operative delivery could be demonstrated. However, a tendency towards an increase in perinatal deaths in the control groups versus the study groups was suggested (Kidd et al., 1985). The relatively small number of pregnancies included in these studies might account for the ambigious endpoints. In a meta-analysis of the available randomized trials on antenatal fetal heart rate monitoring, Mohide and Keirse (1989) pointed out that this diagnostic technique did not have any beneficial effect on perinatal mortality, cesarean section rates, 5-minute Apgar scores, neonatal neurological signs, or admissions to neonatal care units. A sufficiently large, randomized controlled clinical trial, might give answer to the question whether the availability of antenatal fetal heart rate monitoring improves fetal outcome and whether the effectiveness counterbalances its cost. However, such a trial, if conducted, would probably have little impact on clinical management on the basis of the devotion of obstetricians to this diagnostic technique.

\subsubsection{The biophysical profile score}

Another popular method of fetal surveillance is the biophysical profile score developed by Manning et al. (1980). This score is constituted from a proportional assessment of five biophysical variables and, in correspondence with the Apgar score, varies between 0 and 10 . The biophysical profile includes assessment of fetal breathing movements, gross body movements, fetal tone, qualitative amniotic fluid volume using two-dimensional ultrasound and fetal heart rate reactivity using Doppler ultrasound. The combination of these five measurements reduced the false positive rate with regard to prediction of perinatal 
mortality and low 5-minute Apgar scores when compared to any single biophysical test (Manning et al., 1980). In subsequent studies it was suggested that various combinations of less than the original five tests and weighted valuation of the single tests at specific gestational ages might be more practical and equally predictive (Baskett et al, 1984, 1987; Hoddick et al., 1984; Vintzileos et al., 1987; Devoe et al., 1988; Shaw et al., 1990). Also the measurement of placental grading as an additional variable has been suggested (Vintzileos et al., 1983). In prospective trials evaluating the benefit of the biophysical profile versus the nonstress test in the management of high-risk pregnancy, no statistically significant differences could be found in perinatal mortality or morbidity (Manning et al., 1984; Platt et al., 1985). In correspondence with the conclusive remarks concerning fetal heart rate monitoring, a cost-effectiveness analysis might act as a final arbiter.

From the introduction of fetal blood flow velocity waveform measurement in 1977 by FitzGerald and Drumm, many research centres over the world have focused on this subject, presuming that this technique might be a major breakthrough in obstetric care and that it might have beneficial effects on fetal outcome. This section will deal with the biophysical and clinical aspects of fetal Doppler blood flow velocimetry, and with the anatomical and physiological aspects of the fetal circulation. Finally, the objectives of this study will be discussed.

\subsubsection{The Doppler principle}

If a person (sound receiver) is moving towards a sound source, the person will hear the sound at higher frequency than when he is at rest. Conversely, when the person is moving away from the sound source, he will hear the sound at lower frequency. This phenomenon is called the Doppler effect, named after Doppler (1843), who described a frequency shift of light waves in relation to movements of stars and planets. For sound waves, the Doppler effect was experimentelly confirmed by Buys Ballot (1845). The Doppler effect will also occur when the sound source moves towards or away from the receiver and is also valid for sound frequencies above the audio range of the human ear, i.e. 20 $\mathrm{kHz}$. Sound with frequencies higher than $20 \mathrm{kHz}$ is called ultrasound and can be 
generated by piezo-electric crystals which can be mounted in ready-for-use transducers. The ultrasound generated, will have a given, single frequency which depends on the properties of the crystal in the transducer.

Ultrasound with an emission frequency $f_{e}$, emitted towards moving structures, for instance clusters of blood cells, will be dispersed and reflected with a shifted frequency $f_{r}$. The frequency shift is proportional to the velocity and direction of the blood cells. The difference between $f_{r}$ and $f_{e}$ is called the Doppler shift frequency $f_{d}$. As a consequence, $f_{d}$ is proportional to the velocity of the blood cells too, and is expressed in the Doppler formula as:

$$
f_{d}=\frac{2 \cdot f_{e^{*}} v \cos \alpha}{c}
$$

in which,

$\mathrm{v} \cos \alpha=$ the velocity component of the moving blood cells, $\mathrm{c}=$ the transmission velocity of ultrasound in vital tissue $( \pm 1540 \mathrm{~m} / \mathrm{s})$, $\alpha=$ the angle between ultrasonic beams (transducer) and blood flow direction.

This formula is valid under the assumption that the location of the transducer is stable. It further follows from this formula that at right insonation angle, no Doppler shift occurs $\left(\cos 90^{\circ}=0\right)$. The Doppler shift induced by blood flow in large vessels, generated by current diagnostic ultrasound $(1-20 \mathrm{MHz})$ will be within the audio range of the human ear.

\subsubsection{The Doppler shift spectrum}

It should be stressed that reflected waves do not contain one single frequency, but a wide range of frequencies, related to the wide range of velocities present in a blood vessel. Such a range of frequencies is referred to as a frequency spectrum. The pulsatile nature of blood flow in arteries makes the frequency spectrum changing with time, with high maximum Doppler shift frequencies during systole and low maximum Doppler shift frequencies during diastole. The time-related frequency spectrum during a cardiac cycle is called a frequencytime spectrum. Depending on the cross-sectional velocity profile within a blood vessel, maximum frequencies usually originate from the axial centre of the vessel, whereas lower frequencies originate from the paraxial regions. The lowest frequencies usually originate from blood cells streaming along the vessel walls. The analog, frequency shifted (audio) signals, as they come from the Doppler system, can be recorded on magnetic tape for off-line analysis or directly fed into signal processing systems. After amplification, the audio 
signals can be heard as a pulsatile ${ }_{;}$noisy sound. Currently, most research groups use real-time spectrum andlysers for on-line visualization of the frequency-time spectrum. Examples of frequency-time spectra, derived from the blood flow in two different arteries in fetal sheep, are depicted in figure 1.1. This figure stresses the need for registration in quadrature, which implies that forward and reverse velocities are seperately processed: A registration in quadrature enables differentiation between blood flowing towards the transducer and blood flowing away from the transducer. Only bidirectional information of blood flow velocity in a vessel allows reliable determination of a blood flow velocity waveform. Figure 1.2 depicts the maximum envelope curve of one of the frequency-time spectra depicted in figure 1.1. When using the expression blood flow velocity waveform, most authors, inclusive ourselves, refer to the maximum envelope curve.
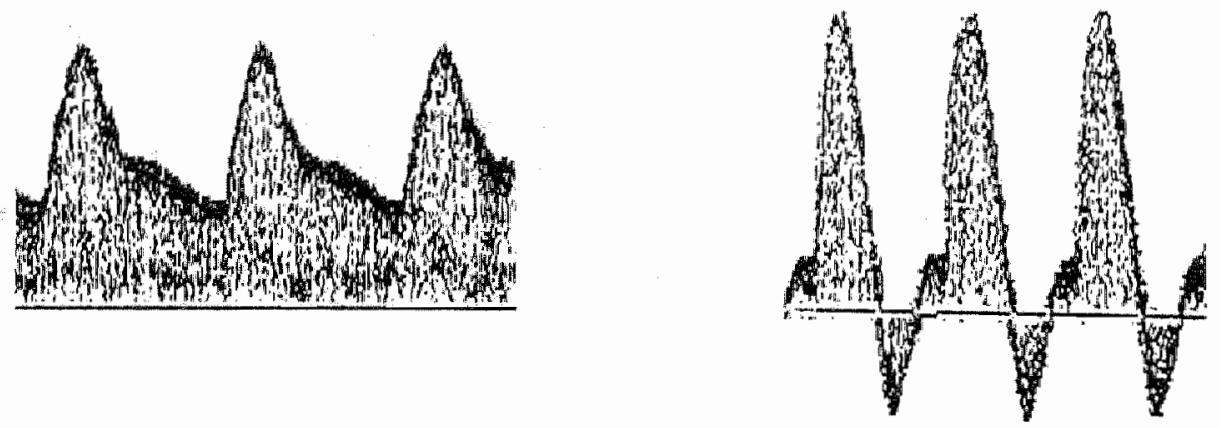

Figure 1.1. Examples of frequency-time spectra, derived from the blood flow in the common umbilical artery (left panel) and the femoral artery (right panel) of fetal sheep.

\subsubsection{Doppler blood flow velocity waveform indices}

Although it is possible to estimate volume blood flow within vessels, this method is out of vogue in obstetric research, for reasons discussed afterwards. Currently, most investigators make use of waveform indices to describe the blood flow velocity waveform. Several indices have been developed, all of them independent of the angle between the blood flow under investigation and the direction of the ultrasound. The most popular indices, the Pulsatility Index (PI) according to Gosling \& King (1975), the A/B or S/D ratio (FitzGerald \& Drumm, 1977; Stuart et al., 1980) and the Resistance Index (RI) according to 


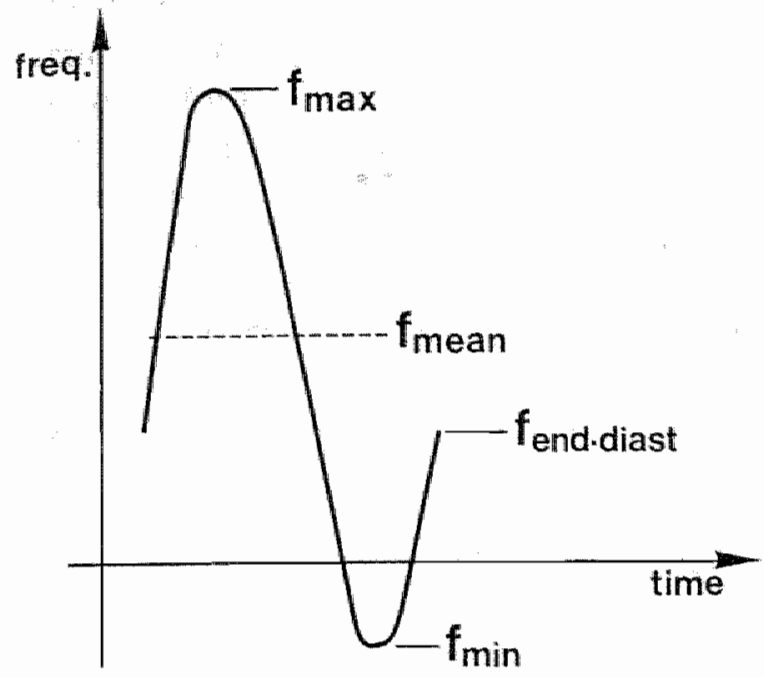

$$
\begin{aligned}
& \text { S/D }=\frac{f_{\text {max }}}{f_{\text {end-diast }}} \\
& P I=\frac{f_{\text {max }}-f_{\text {min }}}{f_{\text {mean }}} \\
& R I=\frac{f_{\text {max }}-f_{\text {end-diast }}}{f_{\text {max }}}
\end{aligned}
$$

Figure 1.2. Maximum blood flow welocity waveform derived from a femoral artery frequencytime spectrum. The frequency shifts used for the calculation of the most frequently used waveform indices are indicated.

Planiol \& Pourcelot (1974) are depicted in figure 1.2. Waveform indices can be calculated either automatically using the built-in software of the spectrum analyser or semi-automatically using additional equipment such as a light-pen. The hemodynamic essence of blood flow velocity waveforms and waveform indices is part of this study and will be discussed later on. In advance it should be mentioned that reduction, absence or reversal of diastolic blood flow velocity relative to systolic blood flow velocity results in an increase in waveform indices. For more extensive descriptions of physical aspects of Doppler blood flow velocity waveforms in general and in obstetrics, the reader is referred to Taylor et al. (1988), Reuwer (1986b) and van Vugt (1988c). For more extensive description of the methodological aspects of our Doppler measurements in fetal sheep, the reader is referred to chapter 2 of this thesis.

\subsubsection{Review of the literature}

Satomura (1959) introduced Doppller ultrasound as a method for investigation of blood flow through peripheral arteries in adults. The introduction of Doppler ultrasound in obstetrics was by Fitzgerald \& Drumm (1977). Using a two-dimensional ultrasound scanner to identify the position of the umbilical 
cord and a continuous wave Doppler transducer (chapter 2), they were able to obtain frequency shifted signals from the umbilical artery and vein, containing continuous information on blood flow velocity throughout the cardiac cycle. They examined pregnancies between 12 and 40 weeks and suggested that this new method should be useful in assessing conditions such as preeclampsia and intrauterine growth retardation. The second report was by McGallum et al. (1978). They obtained blood flow velocity waveforms from the umbilical artery using a real-time $\mathrm{B}$-mode and pulsed Doppler transducer (chapter 2). In succession to Gosling et al. (1971), it was postulated that the umbilical blood flow velocity waveform reflects impedance to blood flow in the fetoplacental circulation. The PI according to Gosling and King (1975) was used for quantitative description of the blood flow velocity waveform. As a result, the umbilical artery PI was considered to be a simple, noninvasive estimation of impedance to fetoplacental blood flow. McGallum et al. (1978) demonstrated that arterial waveforms could be recorded in all patients examined, and that there were differences in the waveform position relative to the zero flow line between normal and some complicated pregnancies.

After these initial reports on the blood flow velocity waveform characteristics in the umbilical circulation, there was a shift of attention to the possibility of volume blood flow measurement (Gill, 1979). This shift was a logical result of the numerous animal studies in which changes in volume blood flow had been demonstrated to be of importance in relation to pregnancy complications comparable with those observed in man. Using a $2 \mathrm{MHz}$ pulsed Doppler system, Gill (1979) was able to calculate volume blood flow in the umbilical vein. In steady conditions, volume flow is expressed by the formula:

$$
Q=v \cdot \pi r^{2}
$$

in which,

$Q=$ volume blood flow,

$v=$ mean blood flow velocity,

$\mathrm{r}=$ mean vessel diameter.

Combination of this formula and that for Doppler shift as explained above results in a new formula comprising the measurements required for calculation of volume flow:

$$
Q=\frac{\pi \cdot r^{2} \cdot f_{d} \cdot c}{2 \cdot f_{e} \cdot \cos \alpha}
$$


It is shown from this formula that determination of wolume blood flow requires the additional measurement of the mean vessel radius and the angle of insonation. Eik-Nes et al. (1980) also measured umbilical vein blood flow and extended their measurements to the fetal aorta, using a so-called duplex system, consisting of a combined $3.5 \mathrm{MHz} \mathrm{B}$-mode linear array scanner and a $2 \mathrm{MHz}$ pulsed Doppler transducer. Umbilical vein blood flow was found to be 110 $\mathrm{ml} / \mathrm{min} / \mathrm{kg}$ fetal weight. Aortic blood flow was found to be $191 \mathrm{ml} / \mathrm{min} / \mathrm{kg}$ and the difference between two measurements did not exceed $10 \%$. More extensive descriptions of volume blood flow measurements in the umbilical vein and fetal thoracic aorta by the same authors seemed promising with regard to investigation of the human fetal circulation (Eik-Nes et al., 1982; Eik-Nes et al, 1984). However, they also distinguished several sources of error, such as measurement of the angle between the ultrasonic beam and blood vessel, measurement of the vessel diameter, position of the sample volume, non-uniform insonation of the vessel, high-pass filtering of the received signal, and the signal-to-noise ratio of the Doppler signal. In a critical report by Gill (1985) it was shown that systematic errors of volume flow measurements were within an acceptable range of 6 percent. However, there was also a considerable amount of random error, mainly attributable to errors in the measurement of the vessel diameter and in the angle of insonation. Also important, but not yet mentioned is the relationship of volume blood flow to estimated fetal weight, since it is relevant to know whether flow is low, though proportional to fetal weight; or whether flow is low even in relation to fetal weight in a growth-retarded fetus. The estimation of fetal weight is hampered by additional sources of error (Hill et al., 1986). As a result, the method requires a certain amount of practice and high demands are made to both instruments and examiners in order to perform reliable measurements. Moreover, since reliabillity increases with repetition and averaging of the measurements, the method is rather time-consuming. Differences in results between examiners up to $50 \%$ have made it questionable whether volume flow estimation can be used for either routine clinical measurements or research purposes (Ruissen et al., 1987a). To date, estimation of volume blood flow in fetal vessels has almost completely been replaced by quantitative assessment of fetal blood flow velocity waveforms.

After the first report by FitzGerald \& Drumm (1977), the same group of investigators serially examined a group of 18 normal pregnancies from 16 weeks gestation untill term (Stuart et al., 1980). They observed a decrease in the A/B-ratio in the umbilical artery with increasing gestational age. It was further concluded that the fetoplacental circulation constitutes a low resistance vascular bed and that conditions which would adversely affect placental perfusion would be detected by this method of investigation. 
From 1984 onwards, the number of reports concerning the subject of fetal blood flow velocity waveforms has exploded and the boom is still going on. First phase studies (Pocock, 1983) included reports on measurement characteristics (Reuwer et al., 1984a; Ruissen et al., 1987a), hemodynamic characteristics (Reuwer et al., 1986a) techinical and methodological aspects (Reuwer et al., 1984a; Ruissen et al., 1987a), location of measurement variation (Maulik et al., 1989), equipment variation (Ruissen et al., 1987b), intra- and inter-observer variability (Nienhuis et al , 1988), fetal physiological variability (Mulders et al., 1986), reference values (Mulders et al., 1987; Pearce et al., 1988), animal studies (Newnham et al., 1987; Trudinger et al., 1987b) and placental pathology studies (Giles et al., 1985; McCowan et al., 1987). The results of these and other first phase studies indicate that the determination of umbilical artery blood flow velocity waveform indices is reliable enough for clinical application and is not hampered by major technical, methodological or economical restrictions. However, the Doppler and processing systems determine the eventual value of the waveform indices and thus limits the possibility to compare results between different centres (Ruissen et al., 1987b). Standardization of equipment would overcome these limitations. Measurements in other fetal vessels, such as the internal carotid arteries (Wladimiroff et al., 1986), middle cerebral arteries (Woo et al., 1987) and renal arteries (Vyas et al., 1989) are more complex and not prone to routine clinical use. More complex than measurement of umbilical artery waveforms too, but possibly relevant, is the measurement of blood flow velocity waveforms in the fetal aorta (Jouppila \& Kirkinen, 1984; Griffin et al., 1984). Nevertheless, many second phase studies were confined to the measurement of umbilical artery blood flow velocity waveform indices. The aim of these studies was to examine the validity of measurement in terms of test sensitivity and specificity, positive and negative predictive values with regard to fetal growth-retardation and fetal distress. Trend-setting and frequently referred studies were those by Reuwer et al. (1984b), Schulman et al. (1984), Fleischer et al. (1985), Trudinger et al. (1985) and Erskine \& Ritchie (1985). Whereas some authors probably correctly focused on umbilical artery Doppler waveform measurement as a monitoring test for fetoplacental circulatory reserve capacity, others ran ahead of proper evaluation of this new test modality and suggested usefulness as a test for fetal well-being and a tool for clinical management. Meanwhile, it has definitely been proven that umbilical artery blood flow velocity waveform analysis is not useful as a screening test for fetal growth-retardation in an unselected population, neither at predefined gestational ages, nor within one week prior to delivery (Mulders et al., 1987; van Vugt et al., 1988; Beattie \& Doman, 1989; Sijmons et al., 1989; Hanretty et al., 1989; Newnham et al., 1990). However, 
more important than prediction of fetal growth, is improvement of both maternal and fetal outcome in comparison with that achieved by current antenatal care. Studies regarded as suggestive for a beneficial effect of availability of umbilical artery blood flow velocity information are those by Trudinger et al. (1987a) and Reuwer et al. (1987).

Trudinger et al. (1987a) reported a randomized controlled trial to investigate the effect of Doppler umbilical artery flow studies on perinatal practice and to assess whether the early identification of the potentially compromised fetus by this method would result in change in timing of delivery and obstetric intervention. The size of the sample-group (300 unselected pregnancies in a hospital population) was calculated on the basis of a one-week difference in gestational age at delivery. It is questionable whether such an outcome criterium is relevant, when mean gestational age appears to be 38 weeks. Improvement of obstetric outcome (less operative deliveries, better fetal outcome parameters) was not regarded as an objective. Although the sample size of the control group was 167, versus a surprisingly lower sample size of the Doppler group (133), there were apparently no differences in percentage occurrence of reasons for admission. In relation to mean birthweight ( $2980 \mathrm{~g}$ in both groups) and the numbers of infants below the 5 th birthweight percentile ( 10 in each group), figures for elective cesarean section (17\% in the control versus $20 \%$ in the Doppler group) appear extremely high and not transferable to obstetric practice in the Netherlands. Moreover, the percentages of induction of labour (almost 50\%) also appear extremely high. The total percentages of cesarean sections were 36 in the control group versus 30 in the Doppler group, not significantly different from each other. The higher incidence of emergency cesarean section in the control group is not supported by a higher incidence of low Apgar scores or low umbilical artery $\mathrm{pH}$ values. No statistically significant differences were observed in the fetal and neonatal outcome parameters used. Nevertheless, the authors concluded that availability of Doppler data resulted in improved neonatal condition.

Reuwer et al. (1987) retrospectively studied 51 pregnancies clinically judged as growth-retarded. All patients were hospitalized. From the time of identification onwards, Doppler studies were performed at weekly intervals until delivery. The results were withheld from the clinicians. Sixteen pregnancies turned out to be normal and all had normal Doppler data (group 1). In group 2A, all 19 fetuses had increased umbilical artery PI values. One fetus had a chromosomal abnormality and two fetuses were considered to have a fatal prognosis. The other 16 fetuses from group 2A were operatively delivered on the basis of fetal distress, of which 15 survived without major handicap. One fetus with a birthweight of 660 grams at 31 weeks died at day 16 from respiratory insuffi- 
ciency. In group 2B (11 fetuses), Doppler data varied between nomal and abnormal, but were not characterized by absence of end-diastolic velocities. Three of the 11 fetuses were electively delivered by repeat cesarean section on the basis of poor obstetric history or growth failure. The other 8 were vaginally and uneventfully delivered. Group 3 consisted of 5 fetuses with normal Doppler data and miscellaneous clinical characteristics.

Whether or not the availability of the Doppler result would have beneficial for pregnancy outcome remains questionable. The fetuses in group 1 were clinically intrauterine growth-retarded but had no signs of perinatal distress. Probably they were appropriate-for-gestational-age at birth. The authors suggest that admission to hospital could possibly have been avoided. Conversely, since severe reduction of end-diastolic velocity might be a rather late sign of fetal placental circulatory incompetence, it might be suggested that these fetuses profited by maternal bed rest and that outcome would have been worse in case of maternal ambulation. The fetuses in group $2 \mathrm{~A}$ were probably severely growth-retarded and thus already recognized as high-risk pregnancies with daily monitoring. Since 15 of 16 fetuses survived without major handicap and one neonatal death was probably inevitable, it remains questionable whether availability of Doppler data would have improved fetal outcome in this group. The authors further suggest that the 3 repeat cesarean sections in group 2B might have been avoided with availability of Doppler data. However, it could also be discussed why "trial of labor" was omitted without any sign of fetal distress, for normal Doppler data are not a prove of good fetal prognosis (Erskine \& Ritchie, 1985).

Reuwer et al. (1987) claimed the need for a test which can identify the growth-retarded fetus truly at risk, since distress would be an indirect and late sign of impairment of the placental circulation. In fact, but indirectly, the authors appeared to suggest the need for a test which can identify conditions of mild or moderate fetal hypoxia. Because the authors themselves gave in that some fetuses with absence of end-diastolic blood flow velocities in the umbilical artery can remain in utero for weeks or months, they essentially underlined that such a Doppler result is not a sign of actual fetal hypoxia, but a sign of evolving fetal hypoxia. 


\subsubsection{Questions raised by the literature}

The results of clinical studies raise diverse questions not yet answered in the preceding sections. Some of these questions are lined up for further evaluation. In the next section will be searched for answers on the basis of data from the literature.

Fetal growth retardation with impending fetal distress is associated with presumed fetal placental circulatory failure. What are the mechanism(s) and underlying cause of this finding?

Is there an anatomical or physiological basis for abnormal findings? Is there a primary disorder in the umbilical or in the uteroplacental circulation?

Application of blood flow velocity waveform indices is based on the assumption that they reflect resistance or impedance to blood flow, constituted in the microvascular bed distally from the place of measurement. Is this assumption justifiable?

Regulation of fetal blood flow distribution is related to regional changes in vascular resistance and depends on regional differences in need of blood supply. During fetal hypoxia, there is a redistribution of blood flow in favor of myocardium, brain and adrenals, and at the expense of viscera, muscles and skin (Peeters et al., 1979). In addition, fetal oxygen delivery is favored by a moderate increase in the fraction of cardiac output perfusing the umbilical circulation (Battaglia \& Meschia, 1986). Is it feasible that waveforms from arteries that supply vascular beds that are highly involved in the regulation of blood flow distribution during hypoxia, provide more information on fetal oxygenation than the umbilical artery blood flow velocity waveform?

\subsubsection{Placental vascular anatomy and pathology}

Since the study described in this thesis is performed in sheep, firstly some comparative aspects of the human and ovine placenta will be discussed. The macroscopic structure of the ovine placenta strongly differs from the compact. discoidal human placenta. The chorio-allantoic sac is studded by $80-100$ cotyledons. All cotyledons together constitute the fetal placenta. The position of the cotyledons in utero is determined by the distribution of complementary structures in the uterine endometrium, known as caruncles. These caruncles are found over the whole surface of the uterus. Caruncles and cotyledones together form placentomes, structures with a diameter of $2-4 \mathrm{~cm}$, the place of maternalfetal exchange. In ovine pregnancy, the umbilical cord is short and it divides into two trunks, each of which contains an artery and a vein. At the level of the 
amnion in the direction of the placentomes, the umbilical arteries divide into a series of major cotyledonary arteries, which on their turn divide into a larger series of minor cotyledonary arteries, which form diverse anastomoses with each other. Each cotyledon receives between two and three cotyledonary arteries, which divide repeatedly and send their terminal branches into the villi. Each villus is supplied by a single, centrally situated artery, which gives off a number of branches, which on their turn supply an extensive capillary plexus beneath the trophoblast in the wall of the villus. The main direction of blood flow through the fetal capillaries is from the tip to the base of the villus. The capillaries drain into two to five collecting veins, which via a circular vein at the base of the villus drain into a cotyledonary vein (Makowski, 1968). Although little detailed literature deals with the diameters in the fetal placental arteriovenous traject in man as well in sheep, some information is available. In both sheep and man, placental capillary diameters seem to vary below 10 micron, whereas the smallest arteries exceed 20 micron (Barcroft \& Barron, 1946; Crawford, 1962; Wimsatt et al., 1962; Giles et al., 1985; Fok et al., 1990).

The microvascular anatomy of the human and ovine fetal placenta shows considerable similarity, wheras, in contrast, the microvascular anatomy of the maternal uteroplacental circulations shows clear differences. The main difference is that the human placenta has a hemochorial structure, which implies that the fetal villi are directly bathed by maternal blood. As a result, the human fetal and maternal circulations are only divided by the fetal capillary endothelium, a small layer of mesodermal tissue and a layer of trophoblast cells. In contrast, the fetal and maternal circulations in sheep are divided by additional layers such as the maternal capillary endothelium, connective tissue and epithelium (Ramsey \& Donner, 1980). In conclusion, the macroscopic as well as the microscopic morphology of the fetal placental circulation in sheep largely resembles that in humans. The maternal placental circulation in man lacks a capillary system and thus essentially differs from that in sheep. From a physiologic standpoint, however, it is apparent that many hemodynamic adaptations are common to placentas of quite different gross structure (Carter, 1975). Several studies were performed to examine the relationship between umbilical artery blood flow velocity waveforms and placental microvascular morphology in human placentas. Studied in an unblinded fashion, Giles et al. (1985) found a marked reduction of the number of small arterial vessels (diameter up to 90 micron) in the tertiary stem villi of the placentas that were characterized by abnormal umbilical artery waveforms. In a number of these abnormal placentas, the authors observed collections of nuclear material that may have represented obliteration of a small artery. The total number of tertiary stem villi did not differ between placentas with normal and abnormal waveforms. The decrease 
in small arterial vessels was explained as either a failure in the development of a sufficient vascular bed or an obliterative process. According to the authors, uteroplacental ischemia and host-versus-graft type of rejection of the fetal placental tissue could possibly give rise to fetal placental vessel obliteration. Contrasting with the hypothesis that uteroplacental ischemia results in fetal placental vascular obliteration is the finding by Scheffen et al. (1989) in guinea pigs that chronic hypoxia results in fetal placental hypercapillarisation. However, the combination of arterial obliteration and capillary sprouting seems, on a teleological basis, rather contradictory.

The findings by Giles et al. (1985) were confirmed by McCowan et al. (1987) and by Bracero et al. (1989). The latter authors suggested a developmental arrest of placental angiogenesis as the most likely explanation for abnormal Doppler findings. Fok et al. (1990) also described increased abnormality of the small arterial vessels in the fetal villi. They found a thickening of the intima and media of the vessels with an outer diameter of 50-149 micron. Probably, this result is not essentially different from the findings of Trudinger et al. (1985), McCowan et al. (1987) and Bracero et al. (1989). Remarkable however, is that reduction of the number of normal small arteries in the tertiary stem villi has never been recognized in relation with fetal growth retardation, in spite of extensive investigation of placental pathology (Brosens et al., 1977; Fox, 1986).

\subsubsection{Doppler blood flow velocimetry and hemodynamics}

In clinical ultrasound studies, the Doppler signal is a consequence of the movement of blood itself, with the Doppler shift frequency dependent on the velocity of this movement. It is natural to ask how velocity might relate to other physiological variables, to pressure, flow rate, and peripheral resistance, for example (Burns, 1988a). In a straight, rigid tube, the relationship between steady pressure and steady flow of a non-viscous fluid is determined by a fluid-mechanical property of the tube, called resistance. This relationship is described by the Poiseuille-equation as:

$$
\text { steadyflow }=\frac{\text { pressure-gradient (over the tube) }}{\text { tubal resistance }}
$$

This equation is frequently used to describe vascular resistance in physiology, although the circulatory system of living beings does not comply with the model of steady flow. The relationship between pulsatile pressure and pulsatile flow is extremely complex. Pulsatile blood flow in arteries is determined by vessel characteristics such as diameter, distensibility, compliance, 
discontinuity and curving; flow characteristics such as turbulence and wave reflections; and blood characteristics such as viscosity and density. With steady flow, the relationship between pressure and flow is described by resistance. With dynamic flow, in the presence of compliance (of the vessels) and inertance (of the blood mass), this relationship is described by impedance (Milnor, 1972; O'Rourke, 1982). Burns (1988a) explained the concept of impedance with regard to Doppler blood flow velocimetry (almost literally) as follows. Any periodic waveform (such as that of the pressure and flow pulses) can be decomposed into a series of sinusoidal components, each a multiple of a single frequency. Considering a single point in the arterial system, the input fluid impedance to the distal circulation is defined as the ratio of pressure to flow of each of these components and hence is a function of frequency. The impedance at zero frequency (i.e. steady flow), is simply the resistance. Reflection will take place, whenever there is a discontinuity in impedance, as usually occurs at a site of arterial branching, such as the aortic bifurcation. Large reflections also occur at the periphery of the circulation. These reflections combine with each other and with the forward-going pulse to modify the pressure pulse waveform. Although the reflections are quite large, their path backwards through arterial branches results in considerable attenuation of amplitude. Investigations have shown that small arteries contribute most to arterial reflections, and of these, arterioles appear to be especially significant (O'Rourke \& Taylor, 1966). If vasoconstriction or vasodilatation is induced in these vessels, the impedance is seen to change. This is a crucial observation for the burgeoning number of Doppler techniques that rely on changes in the shape of the velocity pulse upstream to assess relative impedance of an arteriolar bed. Thus if vasoconstriction occurs, for example, the reflection coefficient increases, and a more pulsatile velocity pulse is observed upstream. At the same time, vasoconstriction increases resistance, reducing the mean, or steady, component of flow. These two factors combine to produce the familiar distinction between time-velocity waveforms associated with low and high distal impedances (figure 3). These waveforms can be quantitatively classified by the waveform indices described previously. Skidmore et al. (1980) demonstrated that of these, the Pulsatility Index was sensitive to changes in distal impedance.

\subsubsection{Regulation of the fetal circulation}

Harvey (1628) provided the first accurate description of the fetal circulation in his "Exercitatio anatomica de motu cordis et sanguinis in animalibus". The recognition of oxygen uptake and carbon dioxide delivery via the fetoplacental 

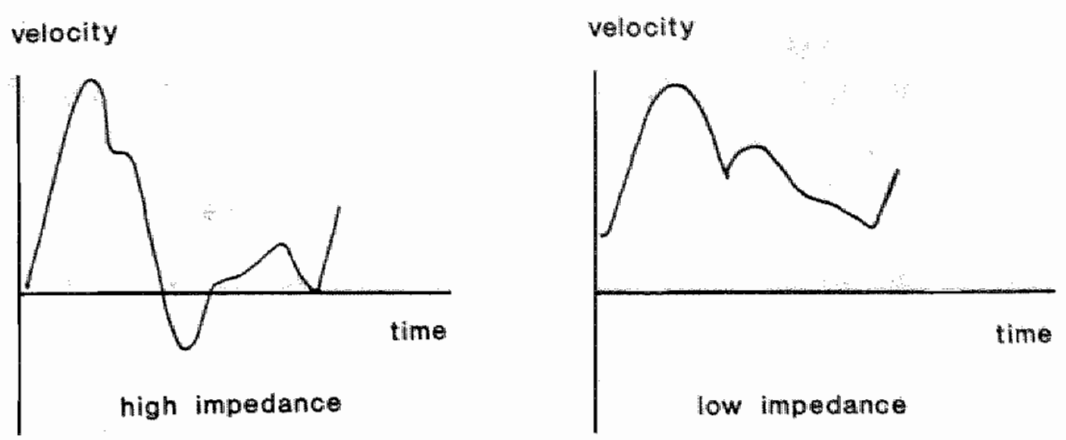

Figure 1.3. Blood flow velocity waveforms during conditions of high and low distal impedance (redrawn from Burns, 1988a).

circulation is ascribed to Zweifel (1876). Attempts to measure blood flow in the umbilical cord were performed by Cohnstein and Zuntz $(1884,1888)$. The first studies on circulatory and respiratory physiology in intact ovine fetuses were performed by Barcroft and coworkers (1946). The results of extensive study in mainly exteriorized ovine fetuses in the fifties and sixties has been summarized by Dawes (1968). Meschia et al. (1967) reported the measurement of umbilical blood flow in unanesthesized fetal sheep in utero using the antipyrine diffusion method. Heymann \& Rudolph (1967) introduced the radionuclide-labeled microsphere method to measure cardiac output distribution in the unanesthesized fetal lambs. The latter method and the electromagnetic flowmeter (Berman et al., 1975) are still the most frequently used techniques for the measurement of blood flows in fetal animals. The development of Doppler ultrasound has now made it possible to study blood flow characteristics in the human fetus without interference with the normal intrauterine environment.

Both the umbilical and fetal systemic circulations are essential parts of the oxygen and nutrient delivery system from placenta to fetal tissues, and conversely, of the carbon dioxide and waste product delivery system from fetal tissues to placenta. The fraction of cardiac output perfusing the placenta must be balanced against the fraction perfusing the fetal body to attain optimal exchange of gaseous and solute substances. Umbilical and systemic venous returns mix to form the output of the fetal heart. The blood ejected by the fetal heart is divided in two blood flows: the flow through the umbilical circulation and the systemic blood flow. With a decrease in blood oxygenation, oxygen delivery is favoured by a moderate increase in the fraction of cardiac output perfusing the umbilical circulation (Battaglia \& Meschia, 1986). The mechanisms by which the fetus maintains the appropriate balance between placental and systemic circulations are not well understood. Several hormones and vaso- 
active substances contribute to the regulation of the fetal circulation, both under normal conditions, and in response to emergency situations such as hypoxia and hemormage (Assali et $a l_{*}, 1978$ ). Hormonal regulation of the vascular system may be particularly important in the fetus because $40 \%$ of fetal cardiac output perfuses the umbilical vasculature which is not under neural control (Berman et al., 1978).

With regard to this study, the regulation of the fetal ovine circulation during hypoxia in the third trimester requires further elucidation. The initial response to hypoxia $\left(\mathrm{PaO}_{2}<15 \mathrm{mmHg}\right.$ ), is a fall in heart rate and a rise in arterial pressure and there is also an increase in heart rate variability (Dalton et al., 1977; Parer et al., 1980). With prolonged hypoxia, the bradycardia becomes smaller and a tachycardia may supervene (Bocking et al., 1988). Arterial pressure remains elevated, as does heart rate variability, unless there is a severe acidosis (Dalton et al., 1977). Chemoreceptor stimulation raises sympathetic efferent activity, which results in a release of catecholamines from the adrenal medulla into the circulation. It is uncertain whether catecholamines are released by a direct hypoxic stimulation of the adrenal medulla (Jones et al., 1988; Cheung, 1989). The main source of plasma epinephrine is the adrenal gland, whereas plasma norepinephrine also originates from the extra-adrenal chromaffin tissue. With fetal maturation, the response of epinephrine to hypoxia becomes more pronounced. The sum effect of efferent neural sympathetic stimulation and circulating catecholamines is a redistribution of cardiac output in favor of brain, heart and adrenals, at the expense of kidneys, gut, carcass and lungs (Peeters et al., 1979; Rudolph, 1984). Umbilical blood flow remains constant or may even increase. The overall effect of arteriolar vasodilatator and vasoconstrictor mechanisms is an increase in mean arterial blood pressure. The effects exerted by humoral release of catecholamines are delayed in comparison with those by neural stimulation. Besides changes in blood flow distribution, catecholamines produce positive inotropic and chronotropic effects on the heart. They act synergistically with the increased sympathetic discharge on the peripheral circulation. Thus fetal heart rate returns to control, or may increase above it, and arterial pressure increases still further (Hanson, 1988). Plasma renin activity also increases in hypoxia in the fetus, so that the increase in blood pressure may in part be due to the resulting rise in angiotensin I and II (Martin et al., 1987). Other substances, such as arginin-vasopressin, prostaglandins, serotonin, 5-hydroxy-tryptamine, bradykinin and others may also have a role in the regulation of the fetal circulation (Berman et al., 1978). However, their role is less clear than that of the catecholamines (Jones \& Robinson, 1975). In contrast with experimental conditions of selective hypoxia, clinical conditions of hypoxia are often associated with hypercarbia, resulting in asphyxia. The 
acute responses to mild asphyxia, as produced by reduction of uteroplacental blood flow, are characterized by an increase or decrease in heart rate (Martin et al. 1979). The difference in the response from that to hypoxia may be due to to a rise in $\mathrm{PaCO}_{2}$, causing an enhanced catecholamine output from the adrenal medulla (Blanco et al., 1984) and a decrease in parasympathetic activity to the heart (Gu et al., 1985).

The diameter changes related to vasodilatation or vasoconstriction in hypoxia or asphyxia result in changes in organ vascular resistance. Fetal asphyxia is associated with increases in splanchnic and musculocutaneous vascular resistance up to $300 \%$ and renal vascular resistance up to $200 \%$ (Yaffe et al., 1987). This raises the suggestion of regional changes in the pulsatility of the blood flow velocity waveform, and as a result, the possibility to noninvasively measure changes in fetal blood flow distribution.

\subsection{OBJECTIVES OF THE STUDY}

The objective of this study was to address various questions raised with regard to the rationale of Doppler blood flow velocimetry in obstetrics. It was pursued to address some basic questions from a pathophysiological point of view, using an animal experimental model. Because of the resemblance between the human and ovine fetal systemic and umbilical circulations, the study was performed in pregnant sheep, for decades the most frequently used model for the study of fetal physiology.

It has been suggested that the umbilical artery waveform might function as a diagnostic test to distinguish between those growth-retarded fetuses at risk for perinatal distress and those not at risk (Reuwer et al., 1987). Indeed, it is reasonable to assume that fetuses with absence of end-diastolic velocities in the umbilical artery waveform are prone to develop hypoxia on the basis of reduced matemal-fetal oxygen delivery. However, if such a condition has developed gradually, it is unknown whether and to which extent, the fetus has adapted to this condition. Although fetuses with absent end-diastolic umbilical artery blood flow can probably not withstand the additional stress of labor, for the individual fetus this remains uncertain beforehand, because the umbilical artery blood flow velocity waveform does not seem to provide information on actual fetal oxygenation. On the basis of musculocutaneous vasoconstriction, it can be hypothesized that the femoral artery Pulsatility Index increases during fetal hypoxemia. A study was designed to examine the effects of hypoxemia, induced by progressively reduced uteroplacental blood flow, on the umbilical and femoral artery blood flow velocity waveforms. 
Since blood flow velocity waveforms are affected by a variety of physiological variables, such as fetal breathing movements and fetal heart rate (Mulders $e t$ $a l$, 1986), it was hypothesized that simultaneous measurement of blood flow velocity waveforms in different vascular beds would provide a measure of the distribution of (impedance to) blood flow and thus, a tool for noninvasive estimation of fetal oxygenation, relatively insensitive to changes in fetal heart rate. This hypothesis was tested by evaluation of the response of simultaneously measured femoral and umbilical artery blood flow velocity waveforms to fetal hypoxemia, induced by reduction of oxygen supply to the matemal sheep.

It has been hypothesized that uteroplacental ischemia induces morphological changes in the fetal placental microcirculation, responsible for abnormal blood flow velocity waveforms in the umbilical arteries (Giles et al., 1985). A study was designed to examine whether prolonged and substantial fetal hypoxemia, induced by progressive embolization of the uteroplacental microcirculation, would result in abnormal umbilical artery blood flow velocity waveforms.

Since abnormality of umbilical artery blood flow velocity waveforms is associated with obliteration (Giles et al., 1985) and/or wall thickening (Fok et al., 1990) of the small arteries in the fetal villi, and probably results from reduced fetoplacental circulatory competence (Reuwer et al., 1986), we examined the effects of selective partial obstruction of the fetal placental circulation. Also the relationships with total umbilical volume blood flow and umbilical vascular resistance were examined.

It has been suggested that management of fetuses with a normal blood flow velocity waveform in the one umbilical artery and an abnormal in the other, should depend on the normal waveform (Trudinger \& Cook, 1987). However, it seems more rationale to be reliably informed on real reduction in umbilical blood flow. In some sheep, we studied the relationship between abnormal waveforms in the peripheral umbilical circulation and total umbilical blood flow versus abnormal waveforms in the proximal fetoplacental circulation and total umbilical blood flow.

Fetal survelllance is mainly based on monitoring of the fetal heart rate. For several purposes, such as investigation of drug effects and fetal adaptation to presumed hypoxia, it would be relevant to be informed on changes in fetal arterial blood pressure. As stated earlier, fetal hypertension is the net result of vasoconstriction and vasodilatation. In hypoxia, most organs beneath the level of the fetal thoracic aorta exert a hypertensive effect on the fetal circulation with an increase in vascular resistance in these organs. In order to get insight in the possibilities for noninvasive estimation of fetal blood pressure, we examined the acute responses of hypertensive drugs on the aorta blood flow velocity waveform above the level of the visceral organs. 


\section{CHAPTER 2}

\section{Methodology and reliability assessment of Doppler flow blood velocity waveform measurements in fetal sheep}

G.J.J.M. Muijsers, C.J. Ruissen, H. Käsemann, W.A. Jager, H. van Huisseling, T.H.M. Hasaart

Submitted for publication

\section{ABSTRACT}

The understanding and interpretation of Doppler blood flow measurements in clinical obstetrics might be improved by experimental studies in animal models. External as well as implantable Doppler tranducers were applied in chronically and acutely instrumented fetal sheep to perform Doppler measurements in various fetal vessels. The reliability of these measurements was evaluated by an inter-examiner variability study. The reliability of measurement proved to be associated with the type of vessel under investigation and with the signal-tonoise ratio of the Doppler registration.

\section{INTRODUCTION}

Fetal artery Doppler blood flow velocity waveform analysis has been recommended as a new method for fetal surveillance in clinical obstetrics (Trudinger \& Cook, 1990). However, little information is available on the pathophysiology of abnormal Doppler findings. Knowledge of the characteristics of fetal blood flow velocity waveforms at various conditions may be improved by animal studies (Nathanielsz et al., 1987). We designed a number of experimental 
studies in pregnant sheep, to uncover the effect of fetal and matemal circulatory changes on the Pulsatility Index (PI) of fetal artery blood flow velocity waveforms. However, an adequate reliability of the measurements still has to be demonstrated before evaluation of the validity is sensible. To obtain reliable waveform index values from vessels in fetal sheep, high demands have to be made upon Doppler signal acquisition, signal processing and waveform index calculation. For extensive descriptions on technical aspects in human fetal Doppler measurements, the reader is referred to Thompson et al. (1986), Ruissen et al. (1987a) and Burns (1988b). Doppler measurements using external or implantable transducers in laboratory animals are hampered by additional methodological problems and by the question of adequate reliability of the measurements. This paper reports basic technical and methodological aspects of animal experimental Doppler measurements as performed in our laboratory. In order to evaluate the reliability of the measurements, we also studied the inter-examiner consistency in determining the PI from fetal arterial blood flow velocity registrations in sheep.

\section{Methodological aspects}

Signal acquisition. When ultrasound is transmitted towards moving blood cells, the ultrasound is dispersed and reflected with a wide range of shifted frequencies. The amount of frequency shift is directly related to the velocity and the direction of the blood cells. On the basis of the wide range of blood flow velocities, present at each particular site of the circulation, the reflected (received) signal from that particular site will contain a wide range of frequencies and amplitudes (Doppler shift spectrum). Signals can be obtained using continuous wave (CW) and pulsed Doppler (PD). An advantage of CW Doppler systems is that they are not limited in the maximum velocity that can be determined. A disadvantage is that, in principle, the origin of the received signal is unknown, since signals from other moving structures along the ultrasound beam may interfere with the signal intended to process. Therefore, $\mathrm{CW}$ Doppler is not appropriate for examination of vessels for which accurate positioning of the sample volume (region from which Doppler signals are to be perceived) is mandatory.

For examination of the umbilical arteries in the free floating umbilical cord, we used, among others, a continuous wave Doppler instrument (Delalande Electronique, Courbevoie, France) with an emission frequency of $4 \mathrm{MHz}$ and a $300 \mathrm{~Hz}$ high-pass filter, the latter to remove low-frequency high-amplitude signals from vessel walls and other slowly moving structures. The transducer 
was handled at the abdominal skin of the standing or lying ewe. The position of the umbilical cord could be assessed using a two-dimensional ultrasound scanner. Waveforms from the umbilical arteries could further be identified by: the frequency of the velocity pulses (which corresponds to the frequency of the pressure pulses), the typicall monophasic pattern, and the presence of reversely directed venous blood flow within the Doppler sample volume (figure 1, upper panel left). Extemal signal acquisition proved to be, obviously more than in human pregnancy, adversely affected by maternal body and breathing movements.

For examination of the umbilical cord arteries at the fetal end, we used a multi-gate pulsed wave Doppler instrument with an emission frequency of 5.24 $\mathrm{MHz}$ and a pulse repetition frequency of $15 \mathrm{kHz}$ (Hoeks, 1982). Implantable transducers with a diameter of $3 \mathrm{~mm}$ were positioned in a polyvinyl cuff under an angle of approximately $45^{\circ}$. The ready-for-use devices were sterilized with ethylene oxide at $80^{\circ}$ Celsius. The fetal lamb was surgically provided with instruments, while the pregnant ewe was under general anesthesia. The Doppler cuffs were positioned around prepared fetal vessels and approximated side to side by two sutures. In order to obtain unhindered transmission of ultrasound, airbubbles between the vessel and the transducer were removed by instilling $0.9 \% \mathrm{NaCl}$ solution. The position of the sample volume as well as the amplitude of the frequency components could be adjusted by range gating (Hoeks, 1982). A major advantage of pulsed Doppler systems is that the depth from which the reflected signals originate can be selected. A problem is that the maximum velocity that can be detected is limited, especially when the distance between transducer and vessel under investigation increases. In practice, this problem appeared of little importance because of the negligible distance between the implanted transducer and the blood vessel and because of the relatively low maximum velocities encountered in the umbilical arteries.

For examination of blood flows with potential high peak velocities, such as the aorta and systemic peripheral arteries we used a single gate pulsed wave Doppler system with an emission frequency of $20 \mathrm{Mhz}$ and a pulse repetition frequency of $62.5 \mathrm{kHz}$ (545C-4 Directional Pulsed Doppler Flowmeter, Bioengineering, University of Iowa, Iowa City, USA). The transducers were also positioned in a perivascular cuff or were directly mounted on a vessel using sterile tissue-glue. Using an emission frequency of $20 \mathrm{MHz}$, Doppler signals can be obtained from one sample volume at a distance between 0 and $10 \mathrm{~mm}$ from the transducer. Since the size of the sample wolume was relatively small $\left( \pm 0.5 \mathrm{~mm}^{3}\right)$, the depth of the sample volume was adjusted to the position from where optimal signals were obtained. Optimal signals usually originate from the axial centre in the vessel. Unfortunately however, the emission direction of 
built-in transducers cannot be adjusted. This introduces the risk of paraxial insonation, which results in sources of error comparable with incorrect depth adjustment (Ruissen et al., 1987a).

Signal processing. Reflected, frequency shifted ultrasound is converted into audio signals with a wide range of frequencies. After processing with a spectrum analyser (Doptek Ltd., Chicester, England), these audio frequencies can be displayed in a visible frequency-time spectrum (figure 2.1). A frequency-time spectrum represents the velocities of clusters of blood cells as a function of time. The frequency-time spectrum can be numerically described by so-called waveform indices. Frequently used waveform indices are the $A / B$ ratio (S/D ratio), the Resistance Index (RI) and the Pulsatility Index (PI) (Thompson et al., 1986; Ruissen et al., 1987a; Burns, 1988a). For monophasic umbilical artery blood flow velocity waveforms, with forward blood flow velocity during the whole cardiac cycle, these indices correlate very well with each other (Mulders et al., 1987), but for biphasic waveform patterns (figure 2.1, upper panel right), the most accurate information is provided by the PI, since this index describes the whole waveform (Ruissen et al., 1987a). The PI, as used in our experiments is defined as the difference between the maximum and minimum frequency shift divided by the mean frequency shift of the maximum envelope curve troughout the cardiac cycle. When using the expression blood flow velocity waveform, we refer to the maximum envelope curve.

Waveform index calculation. Reliable waveform index (PI) calculation requires Doppler signals of adequate quality. In other words, the amplitude of maximum frequencies within the spectrum should be adequate and the amplitude of noise should be low. Noise is generated by the Doppler instrument itself and by other electronic equipment in the environment (figure 1, middle panel right). The amplitude of the signal divided by the amplitude of the noise, is expressed by the signal-to-noise $(\mathrm{S} / \mathrm{N})$ ratio. If the $\mathrm{S} / \mathrm{N}$-ratio is sufficiently high and if the signals are clear-cut, the PI can reliably be calculated on the basis of computerized integration of the blood flow velocity waveform assessed by software within the spectrum analyser. However, PI values from fetal sheep, obtained by automatic assessment of the frequency-time spectrum, empirically prove to be little consistent. Therefore, the blood flow velocity waveform is drawn by an examiner with a light-pen on the monitor of the spectrum analyser. The PI is then calculated using the built-in algorithm of the spectrum analyser. In order to be permitted to draw conclusions from validity studies, the reliability of the light-pen procedure was examined by an inter-examiner consistency study. 
Reliability of measurement. The reliability of measurement can be expressed in a statistical model by the intra-class correlation coefficient of reliability (Fleiss, 1986; Nienhuis et al., 1988). This coefficient of reliability is defined as the quotient of variance due to differences between measured values and the sum of variances due to differences between measured values, systematic (examiner) errors and random (statistical) errors. In short:

$$
\text { reliability }=R=\frac{\left.\sigma^{2} \text { (PI value }\right)}{\left.\sigma^{2}(\text { PI value })+\sigma^{2} \text { (examiner }\right)+\sigma^{2}(\text { error })}
$$

Reduction of systematic errors and random errors thus improves the reliability of measurement. The accuracy performed by the examiner in drawing the maximum envelope curve affects systematic as well as random error. Since noise appears as speckle inside and outside the frequency-time spectrum, differentation between signal and noise depends on the $S / N$ ratio as well as on the discriminatory capacity of the examiner. Using pulsed wave Doppler, particularly the assessment of the systolic peak of the curve can be difficult, due to the little quantity of blood cells with maximum velocities, which results in a low amplitude of high frequencies in the frequency-time spectrum (figure 2.1, middle panel left). On the other hand, a high amplitude of low frequency noise can also disturb proper analysis of the course of the blood flow velocity waveform (figure 2.1, middle panell right). Factors likely to induce systematic measurement errors were particularly observed in recordings of aorta and femoral artery blood flow velocity signals (figure 2.1 , middle panel left). These factors, which in part might be explained by the presence of turbulent blood flow; include a low amplitude of peak systolic frequencies, a decrease of diastolic blood flow velocity beneath the high-pass filter frequency, and biphasicity of the signal, inducing increased numerical variability.

\section{MATERIALS AND METHODS}

From a series of approximately 100 recordings in fetal sheep, a representative sample of 30 recordings from various fetal vessels, but particularly from the umbilical artery, were selected for reliability analysis. The quality of the signals varied widely at subjective assessment of the signals. In order to prevent an artificial increase in variance due to differences between measured values, all recordings were taken during baseline conditions, i.e. prior to experimental manipulations. Some descriptive information on the recordings, such as the origin of the signal, the emission frequency and mode of the Doppler instrument 

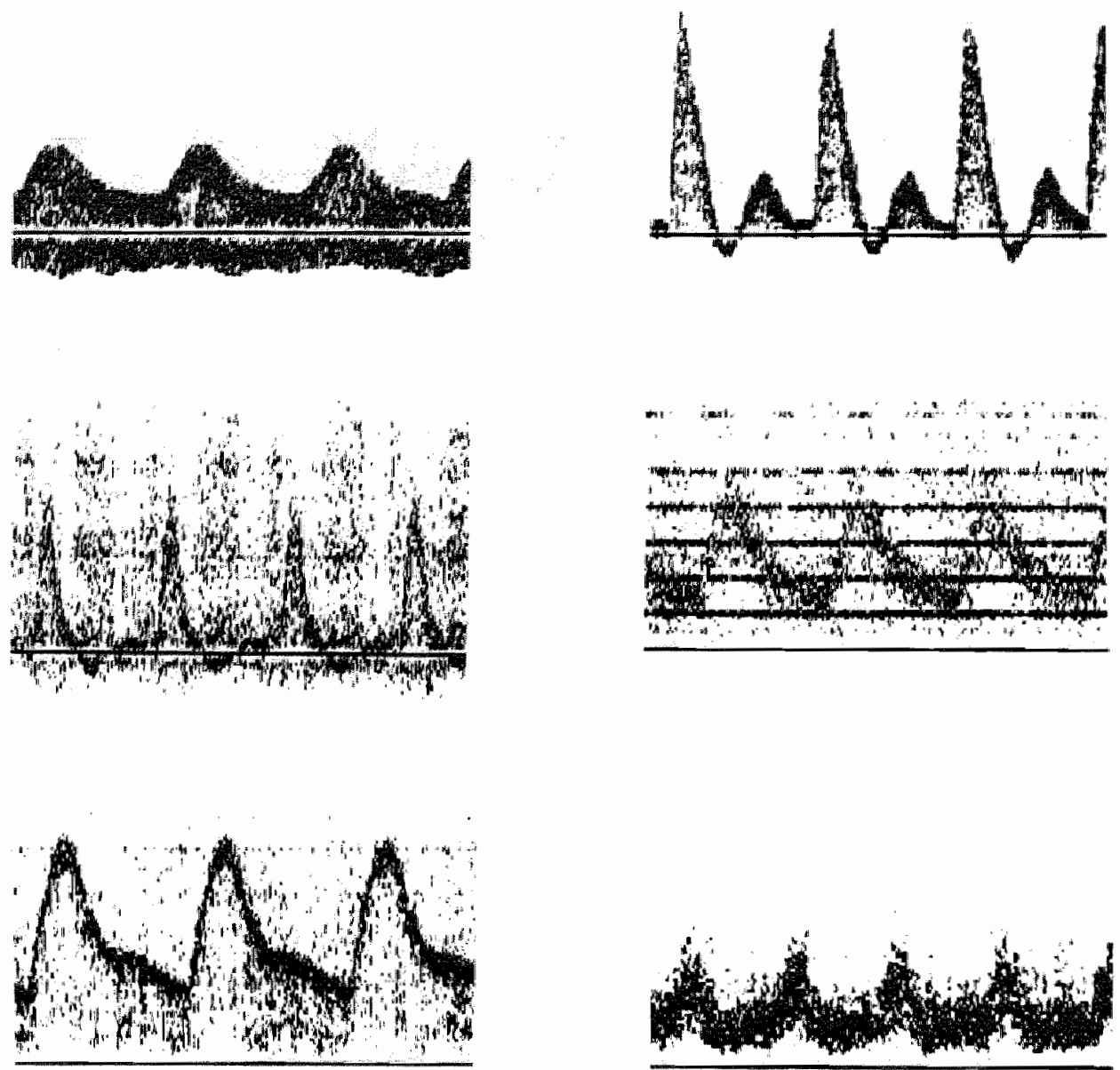

Figure 2.1. Various characteristics of frequency-time spectra in ves sels of the fetal lamb. Upper panel left: arterial and venous umbilical blood flow registered by CW Doppler. Upper panel right: biphasic blood flow pattern in a femoral artery. Middle panel left: unsharp systolic peak and reverse velocities in the sample volume of the fetal femoral artery. Middle panel right: disturbance of an umbilical artery signal by interference (electronic frequency bands). Lower panel left: clear-cut signal from the common umbilical artery obtained by $20 \mathrm{MHz}$ pulsed Doppler ( $\mathrm{S} / \mathrm{N}$-ratio: $18.5 \mathrm{~dB}$ ), Lower panel right: unsharp signal with high amplitude of low fruquencies, and low amplitude of high frequencies ( $S / N$-ratio: $31 \mathrm{~dB})$. 
used, and the $\mathrm{S} / \mathrm{N}$-ratio as determined afterwards, is listed in table 1 . From the original recordings, 30 tracings comprising 50-100 frequency-time spectra were consecutively copied to an audio-tape. Subsequently, the $\mathrm{S} / \mathrm{N}$-ratio of each of the Doppler signals was estimated with a spectrum analyser (Nicolet Scientific Corporation, Northvale, New Jersey, USA).

Three examiners ( $A, B$ and $C$ ), all of them familiar with the principles of Doppler ultrasound, were asked to assess the Doppler signals. All examiners were involved in Doppler ultrasound studies in fetal sheep, but one of them (C) had never been involved in waveform index calculations before. All examiners, independently from each other, visually assessed the maximum curve around five frequency-time spectra within each tracing, to be selected by themselves. This implies that they drew envelope curves around five random spectra with a light-pen on the monitor of the spectrum analyser. The PI values were then automatically calculated, using the built-in algorithm of the spectrum analyser. The mean of five PI values was used for variability analysis. The principles of variability and reliability measurement of quantitative data have extensively been described by Fleiss (1986).

A model was used in which $\sigma^{2}$ (PI value) expresses the variance of the various measured values, $\sigma^{2}$ (examiner) expresses the variance due to systematic examiner errors, and $\sigma^{2}$ (error) expresses the variance due to random (or statistical) errors. The various components of total variance were calculated by performing an ANOVA procedure in SAS statistical software on a VAX computer and by applying the formula for random examiners as described by Fleiss (1986). The respective contributions of variability were then calculated by dividing the variance under investigation by the sum of all variances together. As stated before, reliability(variability due to differences between PI values) is defined as:

$$
\frac{\left.\sigma^{2} \text { (PI value }\right)}{\left.\left.\sigma^{2}(\text { PI value })+\sigma^{2} \text { (examiner }\right)+\sigma^{2} \text { (error }\right)}
$$

the variability due to differences between examiners as:

$$
\frac{\left.\sigma^{2} \text { (examiner }\right)}{\left.\sigma^{2}(\text { PI value })+\sigma^{2} \text { (examiner }\right)+\sigma^{2}(\text { error })}
$$

while the variability due to random error is defined as:

$$
\frac{\left.\sigma^{2} \text { (error }\right)}{\sigma^{2}(\text { PI value })+\sigma^{2}(\text { examiner })+\sigma^{2}(\text { error })}
$$


Reliability and variability were considered in two ways. Firstly, reliability and variability were calculated for 3 groups of 10 signals after ordinal classification of the signals on the basis of their $\mathrm{S} / \mathrm{N}$-ratio. Distinguished were a group containing the 10 signals with the lowest $\mathrm{S} / \mathrm{N}$-ratios (low $\mathrm{S} / \mathrm{N}$ ), a group containing the 10 signals with the highest $\mathrm{S} / \mathrm{N}$ ratios (high $\mathrm{S} / \mathrm{N}$ ), and a group containing the 10 signals with $S / N$-ratios in-between (intermediate $S / N$ ). Secondly and independently of the previous procedure, reliability and variability were calculated after classification of the signals in 4 groups on the basis of their origin and Doppler mode. Thus, 8 signals obtained from an umbilical cord artery using an external $4 \mathrm{MHz}$ continuous wave Doppler transducer (umb $4 \mathrm{MHz}$ ), 6 signals obtained from an umbilical artery at the fetal end using an implanted $5.24 \mathrm{MHz}$ pulsed wave multi-gate Doppler (umb $5 \mathrm{MHz}$ ), 7 signals obtained from various sites in the umbilical artery using an implanted $20 \mathrm{MHz}$ pulsed wave singlegate Doppler transducer (umb $20 \mathrm{MHz}$ ), and 9 signals obtained from other fetal arteries using an implanted $20 \mathrm{MHz}$ pulsed wave singlegate Doppler transducer (non-umb $20 \mathrm{MHz}$ ), were evaluated respectively.

\section{RESULTS}

In rank order of assessment by the examiners, the origin of the signals, the Doppler frequency and mode, the $\mathrm{S} / \mathrm{N}$-ratio, the $\mathrm{PI}$ values as determined by the examiners ( $A, B$ and $C$ ), and the coefficients of variation are listed in table 2.1 . Note that the PI values determined by examiner A were nearly always higher than those determined by examiners B and C, whereas the PI values of the examiners $B$ and $C$ do not show systematic differences.

As shown in tables 2.2 and 2.3 , the variability of the measured PI values exceeds the inter-examiner as well as the random variability for all groups of signals. If classified on the basis of $\mathrm{S} / \mathrm{N}$-ratio (table 2.2), an increase in $\mathrm{S} / \mathrm{N}$ ratio is associated with an increase in reliability and a decrease in the coefficient of variation (COV) as well as the inter-examiner and random variability. If classified on the basis of signal origin and Doppler mode (table 2.3), it appears that the systematic as well as the random errors are lower for signals from the umbilical artery than for signals from non-umbilical fetal arteries. 
Table 2.1. Origin, emission frequency, Doppler mode (continuous or pulsed wave), $\mathrm{S} / \mathrm{N}$ ratio, PI values as determined by 3 examiners and coefficient of variation (COV) from 30 Doppler registrations in fetal sheep.

\begin{tabular}{|c|c|c|c|c|c|c|c|}
\hline \multirow[t]{2}{*}{ signal-origin } & \multirow{2}{*}{$\begin{array}{l}\text { emission } \\
\text { frequency } \\
(\mathrm{MHz})\end{array}$} & \multirow[t]{2}{*}{ mode } & \multirow{2}{*}{$\begin{array}{l}\text { S/N- } \\
\text { ratio }\end{array}$} & \multicolumn{3}{|c|}{ PI values } & \multirow[t]{2}{*}{$\mathrm{COV}$} \\
\hline & & & & ex. A & ex. B & ex. C & \\
\hline 1. abdominal aorta & 20 & PW & 19.5 & 1.36 & 1.34 & 1.22 & 0.058 \\
\hline 2. common umbilical artery & 20 & PW & 18.5 & 1.28 & 1.03 & 1.21 & 0.110 \\
\hline 3. abdominal aorta & 20 & PW & 14.0 & 1.23 & 1.12 & 1.02 & 0.094 \\
\hline 4. common umbilical artery & 20 & PW & 20.5 & 0.77 & 0.69 & 0.74 & 0.055 \\
\hline 5. umbilical cord artery & 5 & PW & 19.0 & 0.77 & 0.65 & 0.67 & 0.092 \\
\hline 6. femoral artery & 20 & PW & 11.5 & 1.92 & 1.84 & 1.35 & 0.182 \\
\hline 7. umbilical cord artery & 4 & $\mathrm{CW}$ & 31.0 & 1.01 & 0.84 & 0.84 & 0.109 \\
\hline 8. umbilical cord artery & 4 & $\mathrm{CW}$ & 35.0 & 0.86 & 0.83 & 0.83 & 0.021 \\
\hline 9. carotid artery & 20 & PW & 20.0 & 1.52 & 1.30 & 1.36 & 0.082 \\
\hline 10. umbilicall cord artery & 5 & PW & 25.0 & 0.61 & 0.45 & 0.44 & 0.191 \\
\hline 11. umbillical cord artery & 5 & PW & 24.0 & 1.12 & 1.10 & 1.07 & 0.023 \\
\hline 12. umbillicall cord artery & 5 & PW & 11.0 & 1.60 & 1.53 & 1.43 & 0.056 \\
\hline 13. common umbilical artery & 20 & PW & 22.0 & 2.20 & 2.00 & 1.98 & 0.059 \\
\hline 14. umbilicall cord artery & 4 & $\mathrm{CW}$ & 21.0 & 0.72 & 0.64 & 0.73 & 0.070 \\
\hline 15. abdominal aorta & 20 & PW & 15.0 & 1.47 & 1.29 & 1.23 & 0.094 \\
\hline 16. external iliac artery & 20 & PW & 18.5 & 1.27 & 1.10 & 1.05 & 0.101 \\
\hline 17. common umbilical artery & 20 & PW & 17.0 & 1.15 & 1.11 & 1.06 & 0.041 \\
\hline 18. umbilical cord artery & 20 & PW & 17.0 & 0.99 & 0.89 & 0.89 & 0.063 \\
\hline 19. umbilical cord artery & 20 & PW & 8.5 & 1.05 & 0.95 & 1.00 & 0.050 \\
\hline 20. femoral artery & 20 & PW & 16.0 & 2.12 & 1.83 & 1.89 & 0.087 \\
\hline 21. femoral artery & 20 & PW & 28.0 & 1.32 & 1.43 & 1.32 & 0.049 \\
\hline 22. umbilical cord artery & 4 & $\mathrm{CW}$ & 21.0 & 0.76 & 0.69 & 0.66 & 0.073 \\
\hline 23. umbillical cord artery & 4 & $\mathrm{CW}$ & 12.5 & 1.36 & 1.32 & 1.17 & 0.078 \\
\hline 24. umbillical cord artery & 4 & $\mathrm{CW}$ & 16.0 & 0.82 & 0.67 & 0.67 & 0.120 \\
\hline 25. umbilical cord artery & 4 & $\mathrm{CW}$ & 17.5 & 0.90 & 0.80 & 0.75 & 0.093 \\
\hline 26. umbilical cord artery & 5 & PW & 14.0 & 1.06 & 0.92 & 0.84 & 0.118 \\
\hline 27. external iliac artery & 20 & PW & 19.5 & 2.16 & 1.73 & 2.03 & 0.112 \\
\hline 28. umbilical cord artery & 20 & PW & 29.0 & 0.94 & 0.85 & 0.86 & 0.056 \\
\hline 29. umbilical cord artery & 5 & PW & 12.0 & 0.97 & 0.84 & 0.87 & 0.076 \\
\hline 30. umbilical cord artery & 4 & $\mathrm{CW}$ & 19.5 & 1.26 & 1.24 & 1.25 & 0.008 \\
\hline
\end{tabular}


Tuble 2.2. Signal-to-noise ratio, coefficient of variation (COV), reliability and variability in 3 groups of Doppler signals, classified on the basis of $S / N$-ratio (low, intermediate and high S/N-ratioss).

\begin{tabular}{llll}
\hline & $\begin{array}{l}\text { low } \mathrm{S} / \mathrm{N} \\
(10 \text { signals })\end{array}$ & $\begin{array}{l}\text { intermediate } \mathrm{S} / \mathrm{N} \\
(10 \text { signals })\end{array}$ & $\begin{array}{l}\text { high } \mathrm{S} / \mathrm{N} \\
(10 \text { signals })\end{array}$ \\
\hline $\begin{array}{lll}\text { mean S/N tatio } \pm \mathrm{SD}(\mathrm{dB}) \\
\text { range of S/N-ratios (dB) }\end{array}$ & $8.5-16.0$ & $17.0-20.0$ & $20.5-35.0$ \\
mean COV & 0.096 & 0.076 & 0.071 \\
reliability & & & $0.9 .6 \pm 1.1$ \\
(imter-signal variability) & 0.89 & 0.92 & 0.98 \\
inter-examiner variability & 0.06 & 0.04 & 0.01 \\
random variability & 0.05 & 0.04 & 0.01 \\
\hline
\end{tabular}

Table 2.3. Signal-to-noise ratio, average PI walue, coefficient of variation (COV), reliability and variability in 4 groups of Doppler signals, classified on the basis of origin (umbilical versus non-umbilical arteries) and Doppler mode (4 MHz CW, $5 \mathrm{MHz} \mathrm{PW}, 20 \mathrm{MHz}$ PW).

umb $4 \mathrm{MHz}$ umb $5 \mathrm{Mhz}$ umb $20 \mathrm{Mhz}$ non-umb $20 \mathrm{MHz}$ ( 8 signals $) \quad(6$ signals $) \quad(7$ signals $) \quad(9$ signals $)$

\begin{tabular}{|c|c|c|c|c|}
\hline mean $\mathrm{S} / \mathrm{N}$ ratio $\pm \mathrm{SD}(\mathrm{dB})$ & $21.7 \pm 7.6$ & $17.5 \pm 6.1$ & $18.9 \pm 6.2$ & $18.0 \pm 4.7$ \\
\hline range of $\mathrm{S} / \mathrm{N}$-ratios $(\mathrm{dB})$ & $12.5-35.0$ & $11.0-25.0$ & $8.5-29.0$ & $11.5-28.0$ \\
\hline average PI value & 0.90 & 0.94 & 1.13 & 1.47 \\
\hline mean COV & 0.072 & 0.093 & 0.062 & 0.095 \\
\hline \multicolumn{5}{|l|}{ reliability } \\
\hline (inter-signal variability) & 0.91 & 0.95 & 0.95 & 0.72 \\
\hline inter-examiner variability & 0.05 & 0.04 & 0.04 & 0.17 \\
\hline random variability & 0.04 & 0.01 & 0.01 & 0.11 \\
\hline
\end{tabular}




\section{DISCUSSION}

It is concluded from this study that the inter-examiner consistency in assessing Doppler recordings from fetal sheep is associated with the origin as well as the $\mathrm{S} / \mathrm{N}$-ratio of the signal. Only a minor role however, can be attributed to the $\mathrm{S} / \mathrm{N}$-ratio. Even in registrations with a low $\mathrm{S} / \mathrm{N}$-ratio beneath $16 \mathrm{~dB}$ (table 2.2), the differences between the waveforms proved to be much bigger than the differences in assessment by the examiners. In general it appears that, independently of the Doppler system used, registrations originating from the umbilical arteries can be reliably assessed. This assumption concurs with the results of the study by Gudmundsson et al. (1990). Although the number of potential variables in that study was higher and the aim differed from the one reported here, the coefficient of variation of the umbilical artery PI proved to be lower than 12 percent, being a critical value in the assessment of diagnostic tests (England, 1975).

On the basis of their higher bandwidth in comparison with PD, CW Doppler signals may be characterized by a high signal amplitude, although the envelope curve may be difficult to assess (figure 2.1, lower panel right). In contrast with signals obtained by CW Doppler systems, PD signals are often characterized by a high amplitude of maximum velocities and a low amplitude of lower velocities, which implies that the sample volume is positioned in the axial centre of the vessel (figure 2.1 , lower panel left). These spectra are characterized by a high signal amplitude and a high signal resolution. Spectra with a high amplitude of lower velocities and a low intensity of maximum velocities are characterized by a high signal amplitude but, in contrast, by a low signal resolution (figure 2.1, lower panel right). A low intensity of maximum velocities was particularly observed during peak systole in vessels such as the fetal aorta and femoral artery (figure 2.1, middle panel left). In the fetal aorta, this might be clue to the presence of turbulent blood flow, resulting in peak velocities throughout the cross-sectional vessel area. In the femoral artery, it is assumed that paraxial insonation may have resulted in a low amplitude of peak velocities. Such a poor resolution of the systolic peak might result in an over- or underestimation of the position of the systolic peak, and thus, adversely affect correct PI calculation. This phenomenon might in part explain the increase in systematic and random variability in the assessment of non-umbilical Doppler signals at average $S / \mathrm{N}$ ratio, as shown in table 2.3 . 
The results of this study emphasize some of the demands to be considered for reliable Doppler measurements. It is shown that examiners are fairly to highly consistent in assessing the envelope curve of blood flow velocity spectra from fetal sheep, suggesting that the quality of the registrations meets the requirements for reliable measurements. 


\section{CHAPTER 3}

\section{The response of the umbilical and femoral artery Pulsatility Indices in fetal sheep to progressively reduced uteroplacental blood flow}

G.J.J.M. Muijsers, T.H.M. Hasaart, C.J. Ruissen, H. van Huisseling, L.L.H. Peeters, J. de Haan

Journal of Developmental Physiology, in press

\section{ABSTRACT}

Fetal artery Doppler velocimetry may provide noninvasive information on the state of fetal oxygenation. It was hypothesized that during decreasing fetal oxygenation, the pulsatility index in the femoral artery will increase, whereas the pulsatility index in the umbilical artery will not change. Decreasing fetal oxygenation was induced in ten chronically-instrumented fetal sheep by progressive occlusion of the maternal common internal iliac artery. The pulsatility index in the umbilical artery was serially measured in six fetuses (group $I, n=6$ ) and the pulsatility index in the femoral artery was serially measured in four fetuses (group II, $n=4$ ). Fetal arterial oxygen content decreased by $72 \%$ in group I and by $79 \%$ in group II. Fetal heart rate did not change. Fetal blood pressure increased by $11 \%$ in group I and by $15 \%$ in group II. The umbilical artery pulsatility index (group I) did not significantly change during decreasing fetal oxygenation, whereas the femoral artery pulsatility index (group II) increased by $140 \%$. It is concluded that progressively reduced uteroplacental blood flow 
results in fetal hypoxemia, which is associated with an increased pulsatility index in the femoral artery, while the pulsatility index in the umbilical artery does not change.

\section{INTRODUCTION}

Indices of the umbilical artery blood flow velocity waveform are supposed to provide direct and essential information on the adequacy of the fetoplacental circulation (Reuwer et al., 1987). Moreover, Doppler measurements in the umbilical artery would allow recognition of potential fetal compromise and facilitate improved fetal management (Trudinger et al., 1987a). However, in the recognition of fetal compromise, the predictive value of umbilical artery blood flow velocimetry has not proven to be higher than that of fetal heart rate monitoring (Trudinger et al., 1986). Results from clinical Doppler studies indicate that reduction, absence or reversal of diastolic blood flow velocity in the umbilical artery is associated with fetal hypoxemia (Ferrazzi et al., 1988). These phenomena appear to be associated with morphological changes in the placental microcirculation, rather than with fetal circulatory adaptation mechanisms to shortlasting hypoxemia (Giles et al., 1985; van Huisseling et al., 1989).

In various species, the circulation of the near-term fetus responds to hypoxemia with a series of changes triggered by chemoreceptor stimulation (Mott, 1982). These changes include hypertension, bradycardia and a redistribution of blood flow away from intestines, kidneys, skeletal muscle and skin, and in favor of myocardium, brain and adrenals. Changes in umbilical blood flow and umbilical vascular resistance secondary to hypoxemia are either small or inconsistent, unless bradycardia and/or asphyxia occur (Cohn et al., 1974; Peeters et al., 1979; Cohn et al., 1985; Yaffe et al., 1987).

The Pulsatility Index of the umbilical artery blood flow velocity waveform (umbilical PI) is assumed to reflect resistance or impedance to umbilical blood flow (FitzGerald \& Drumm, 1977; McGallum et al., 1978). From data on umbilical vascular resistance and fetal hypoxemia in a condition without bradycardia it may be expected that the umbilical PI will change little in response to fetal hypoxemia (Yaffe et al., 1987). In contrast, in the femoral artery, which predominantly supplies skeletal muscle and skin, the PI should increase in response to hypoxemia (Dawes et al., 1968).

The aim of this study was to evaluate the short-term response of the Pulsatility Indices in the umbilical and femoral arteries to moderate and severe fetal hypoxemia, induced by reduced uteroplacental blood flow. Since the Pulsatility 
Index is affected by changes in fetal heart rate, uteroplacental blood flow was gradually reduced over several hours, in order to prevent acute vagally mediated fetal bradycardia (Mulders et al., 1986; van Huisseling et al., 1989).

\section{MATERIALS AND METHODS}

Surgery. The study was performed in ten pregnant sheep of the Dutch Texel breed. Surgery was performed under general anesthesia between the 108 th and 111 th day of gestation (term 147 days). Anesthesia was induced with pentobarbital and maintained with $1 \%$ halothane in a $2: 1$ mixture of nitrous oxide and oxygen. Prior to surgery, the ewes received 1 gram of ampicillin intravenously. After opening the abdomen through a paramedian incision, an inflatable balloon occluder (inflatable volume: $\pm 2.0 \mathrm{cc}$ saline) was placed around the maternal common internal iliac artery. The uterus was incised over the fetal pelvis and the fetal hindlimbs were exteriorized. A polyvinyl catheter (outer diameter 1.6 $\mathrm{mm}$, inner diameter $0.8 \mathrm{~mm}$ ) was inserted into one of the femoral arteries and advanced to a level just above the aortic trifurcation. A $20 \mathrm{MHz}$ pulsed Doppler transducer, fixed in a polyvinyl perivascular cuff under a $45^{\circ}$ angel, was placed on the contralateral femoral artery and on one of the umbilical arteries near the umbilicus. The umbilical arteries were dissected free at the abdominal entry of the cord and covered again with Wharton's jelly after placement of the Doppler device. ECG electrodes were implanted subcutaneously and a polyvinyl catheter for measurement of amniotic pressure was sutured to the fetal skin. The catheters, Doppler cables and ECG wires were exteriorized through a skin incision in the ewe's flank. The guidelines for the care and use of the animals, approved by the local Animals Medical Ethics Committee, were followed.

Measurements. Fetal aortic pressure and intra-amniotic pressure were determined with the zero point at the level of the ewe's spine. Fetal blood pressure was defined as the pressure difference between fetal aorta and amniotic cavity. All pressure- and bioelectric signals were fed into amplifiers (Hewlett Packard 8800 Series, Hewlett Packard Company, Andover, Massachusetts), displayed on monitor, recorded on eight-channel strip-chart recorder and stored on magnetic tape. Mean values of fetal heart rate and fetal blood pressure were calculated by computer over 10 -seconds periods. Fetal arterial oxygen content (fetal $\left[\mathrm{O}_{2}\right]_{\mathrm{a}}$ ) was determined from the hemoglobin concentration and the oxygen saturation (OSM2 Hemoximeter, Radiometer, Copenhagen). Blood flow velocity waveforms in the umbilical and femoral arteries were obtained by means of the implanted $20 \mathrm{MHz}$ pulsed Doppler transducers (545C-4 Directional Pulsed Doppler Flowmeter, University of Iowa, Iowa City). The frequency shifted 
phasic signals were stored in quadrature on analog audiotape and transformed by a spectrum analyser (Doptek spectrum analyser, Doptek Ltd., Chicester). For theoretical and practical reasons, numerical description of the blood flow velocity waveform by means of the Pulsatility Index (PI) according to Gosling et al. (1971) has become the most commonly employed method of fetal artery Doppler velocimetry (Thompson et al., 1986; Ruissen et al., 1987a). The PI, as used in our experiments, is defined as the difference between the maximum and minimum frequency shift divided by the mean frequency shift of the maximum envelope curve throughout the cardiac cycle (Thompson et al., 1986). Parameter calculations were performed off-line by drawing the maximum envelope curves of the frequency-time spectra with a hand-guided light-pen (Ruissen et al., 1987a).

Experiments. The animals were allowed to recover for 3 or 4 days after surgical instrumentation. Gestational age at the time of the experiments ranged from 111 to 115 days. In spite of the fact that all ten fetal sheep were equipped with Doppler devices on both one of the femoral and one of the umbilical arteries, none of them gave adequate recordings from both devices simultaneously due to weak signal-to-noise ratios from one of the signals or breaking-down of one of the Doppler wires. Therefore, the original group of ten fetal sheep was divided in into two independent groups for subsequent analysis. Group I consisted of six fetuses with recordings of umbilicall artery blood flow velocity waveforms. Group II consisted of four fetuses with recordings of femoral artery blood flow velocity waveforms. Baseline values of fetal $\left[\mathrm{O}_{2}\right]_{\mathrm{a}}$, heart rate, blood pressure, femoral PI or umbilical PI were measured over a 1-hour period prior to manipulation. Subsequently, a gradual reduction in uterine blood flow was induced by stepwise, maintained increments in the degree of inflation of the occluder around the common internal iliac artery. After each increment in balloon inflation the fetus was allowed to adapt to the new state for 10 minutes. Fetal heart rate, blood pressure, femoral PI or umbilical PI and $\left[\mathrm{O}_{2}\right]_{\mathrm{a}}$ were then measured simultaneously. Total balloon inflation was reached in approximately 20 to 30 inflations of \pm 0.07 to $\pm 0.10 \mathrm{cc}$ saline each, and thus accompanied by a proportional number of measurements per fetus. The balloon was deflated at once, as soon as complete occlusion was reached or when acute bradycardia occurred. The measurement of fetal heart rate, blood pressure, femoral artery or umbilical PI and $\left[\mathrm{O}_{2}\right]_{\mathrm{a}}$ was continued for one hour after release of the occluder.

Calculations and data analysis. The femoral and umbilical PI values were calculated as mean values, averaged over 5 consecutive cardiac cycles. Mean PI values with a coefficient of variation of more than $10 \%$ were discarded. Fetal heart rate and blood pressure were averaged over 10 -seconds periods using a 
personal computer program. Within each of the fetal sheep, a linear regression analysis was performed using all data obtained of fetal heart rate, blood pressure, femoral or umbilical PI as dependent variables, and all data obtained of fetal $\left[\mathrm{O}_{2}\right]_{\mathrm{a}}$ as independent variables. Subsequently, the slopes of linear regression were tested by two-tailed t-test. Average slopes were considered to be statistically significant when the interval of reliability exceeded $0.95(p<0.05)$. In addition, the average changes in $\left[\mathrm{O}_{2}\right]_{\mathrm{a}}$, fetal heart rate, fetal mean arterial pressure, umbilical PI and femoral PI from baseline condition to deepest hypoxemia were calculated and tested by paired-comparisons $\mathrm{t}$-test.

\section{RESULTS}

Reduction in uteroplacental blood flow by stepwise increasing occlusion of the common internal iliac artery resulted in a gradual decrease in fetal oxygenation. Fetal $\left[\mathrm{O}_{2}\right]_{\mathrm{a}}$ decreased from $2.91( \pm 0.32) \mathrm{mM}$ to $0.81( \pm 0.25) \mathrm{mM}(\mathrm{p}<$ $0.0001)$ in group I and from $3.07( \pm 0.27) \mathrm{mM}$ to $0.64( \pm 0.22) \mathrm{mM} \quad(\mathrm{p}<0.0001)$ in group II (means $\pm \mathrm{SD}$ ). Base excess, $\mathrm{pH}$ and $\mathrm{PaCO}_{2}$ were determined in eight fetuses. Base excess decreased from $-1.0( \pm 2.0) \mathrm{mM}$ to $-5.4( \pm 2.7) \mathrm{mM}(\mathrm{p}<$ $0.01) ; \mathrm{pH}$ decreased from $7.36( \pm 0.04) \mathrm{mM}$ to $7.22( \pm 0.08) \mathrm{mM}(\mathrm{p}<0.01)$ and $\mathrm{PaCO}_{2}$ increased from $44.0( \pm 4.4) \mathrm{mmHg}$ to $57.1( \pm 15.4) \mathrm{mmHg}(\mathrm{p}<0.05)$. In group I and group II, the distributions of the corresponding physiological and biochemical parameters appeared to be normal and did not differ between both groups (Fisher-tests:n.s.). A mean coefficient of variation of less than $5 \%$ was found for both umbilical PI and femoral PI.

Linear regression analysis between fetal heart rate, fetal mean arterial pressure, umbilical $\mathrm{PI}$, femoral $\mathrm{PI}$ and $\left[\mathrm{O}_{2}\right]_{\mathrm{a}}$ yielded the following results: in both group I and group II, the stepwise common internal iliac artery occlusion resulted in inconsistent changes in fetal heart rate (figure 3.1, upper panels) and an increase in fetal mean arterial pressure (figure 3.1 , middle panels). In group I, umbilical PI changed inconsistently (figure 3.1, left lower panel), whereas in group II, the femoral PI increased consistently (figure 3.1, right lower panel).

Table 3.1 lists the average coefficients of linear regression and their $\mathrm{p}$-values. In both groups, the mean change in fetal heart rate with stepwise common internal iliac artery occlusion was insignificant. In contrast, in both groups, fetal mean arterial pressure increased by more than $2 \mathrm{~mm} \mathrm{Hg}$ per $1 \mathrm{mM}$ decrease in $\left[\mathrm{O}_{2}\right]_{\mathrm{a}}$. The average change in umbilical PI was insignificant, whereas that in femoral PI was more than 1 unit per $1 \mathrm{mM}$ decrease in $\left[\mathrm{O}_{2}\right]_{\mathrm{a}}$. 

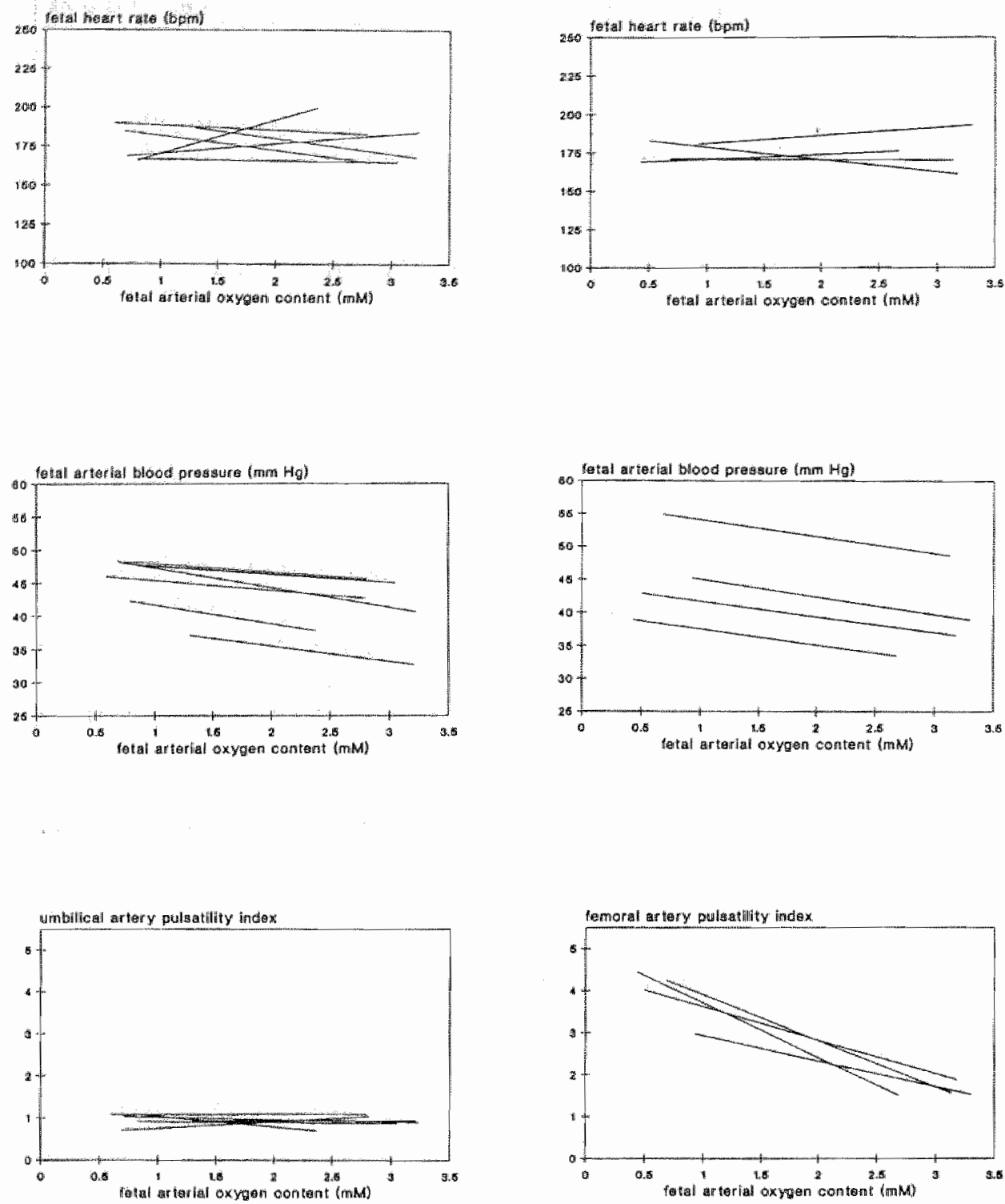

Figure 3. 1. Linear regression lines expressing the relarionship between the various cardiovascular variables and fetal arterial oxygen content in the 6 fetuses from group I (left panels) and the 4 fetuses from group II. 
Table 3.1. Mean coefficients (slopes) of linear regression in group I and group II. A mean coefficient expresses the mean change in fetal heart rate (bpm), mean arterial pressure ( $\mathrm{mmHg}$ ) and $\mathrm{PI}$ per $1 \mathrm{mM}$ change in $\left[\mathrm{O}_{2}\right]_{a}$. Statistical significance of the mean coefficients was tested by two-tailed t-test.

\begin{tabular}{|c|c|c|c|c|c|c|}
\hline \multirow[b]{2}{*}{ regressed variables } & \multicolumn{3}{|c|}{ GROUP I $(n=6)$} & \multicolumn{3}{|c|}{ GROUP II $(n=4)$} \\
\hline & slope & SEM & p-value & slope & SEM & $\mathrm{p}$-value \\
\hline fetal heart rate vs. $\left[\mathrm{O}_{2}\right]_{\mathrm{a}}$ & 0.79 & 4.84 & n.s. & -2.47 & 2.58 & n.s. \\
\hline mean arterial pressure $v s .\left[\mathrm{O}_{2}\right]$ & -2.15 & 0.29 & $<0.001$ & -2.50 & 0.07 & $<0.001$ \\
\hline umbilical artery PI ws. $\left[\mathrm{O}_{2}\right]_{a}$ & -0.03 & 0.05 & n.s. & & & \\
\hline
\end{tabular}

femoral artery PI vs. $\left[\mathrm{O}_{2}\right]_{\mathrm{a}}$

$-1.02 \quad 0.19<0.02$

In order to provide insight in the degree of cardiovascular stability during the experiment, the values of the cardiovascular variables corresponding with the values of fetal $\left[\mathrm{O}_{2}\right]_{\mathrm{a}}$ at baseline condition, nearest to $2.75,2.50,2.25,2.00,1.75$, $1.50,1.25$ and $1.00 \mathrm{mM}$ respectively and at deepest hypoxemia, from each fetus in both sample groups were determined. The mean values, standard deviations, and range values of the biophysical variables were calculated for each of the aimed intervals of fetal $\left[\mathrm{O}_{2}\right]_{a}$ and plotted in figure 3.2 .

The mean values of the variables measured during baseline condition and during deepest hypoxemia are listed in table 3.2 . Fetal $\left[\mathrm{O}_{2}\right]_{\text {a decreased by } 72 \%}$ in group I and by $79 \%$ in group II. Fetal mean arterial pressure increased consistently by $11 \%$ in group I and by $15 \%$ in group II. The umbilical PI did not change appreciably, whereas the femoral PI increased by $150 \%$ during a comparable decrease in $\left[\mathrm{O}_{2}\right]_{a}$.

Stepwise linear regression procedure in group I between the umbilical PI and the other variables demonstrated that the umbilical PI covaried significantly with fetal heart rate in each of the fetuses $(0.0001<\mathrm{p}<0.05)$. The average increase in umbilical PI was 0.0058 unit, with an decrease in fetal heart rate of 1. bpm $(\mathrm{p}<0.01)$. The correlation coefficient of the regression between the umbilical PI and fetal heart rate varied between -0.42 and $-0.61 \quad(0.01<\mathrm{p}<$ $0.0001)$. 

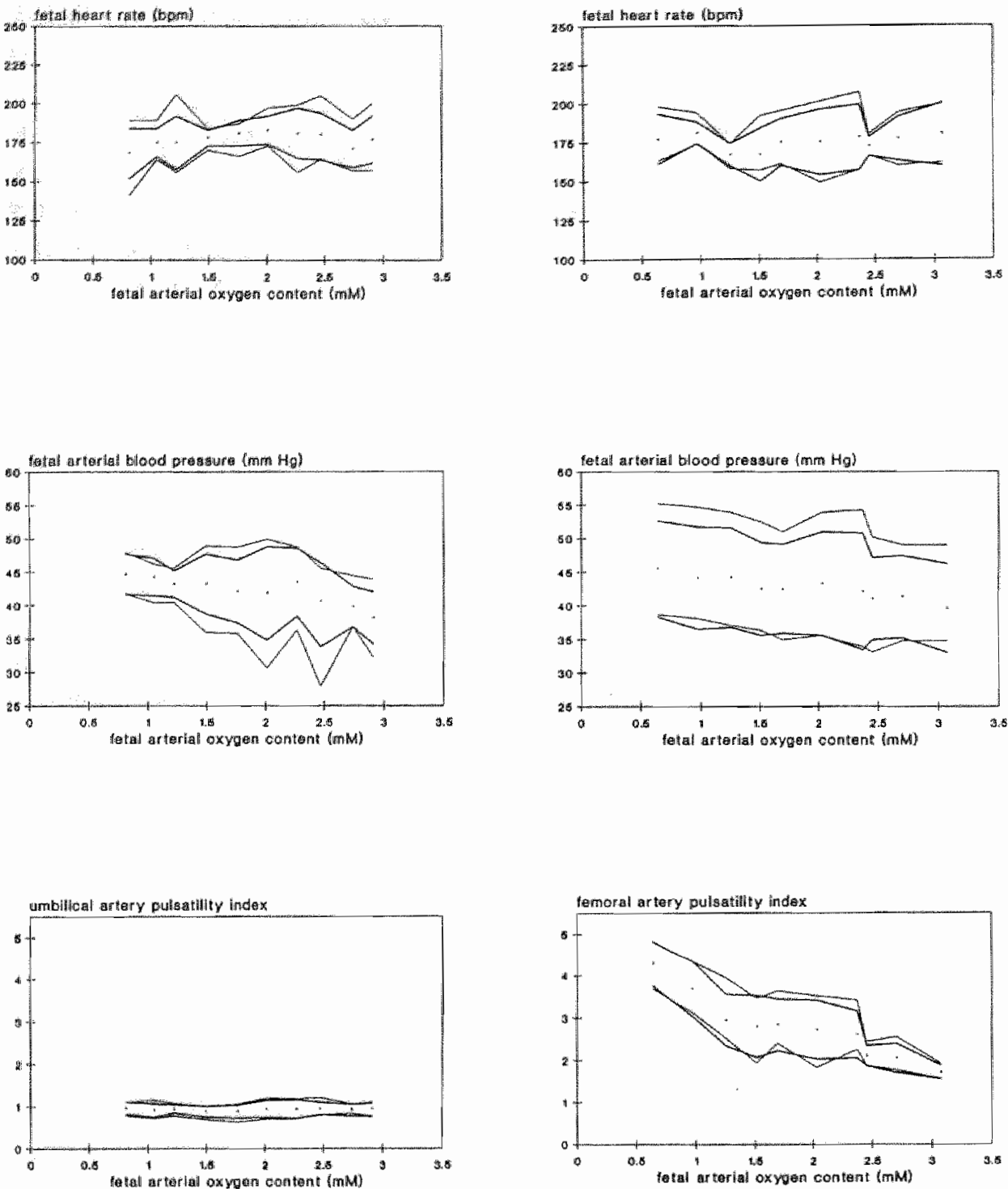

Figure 3.2. Course of the cardiovascular variables during the experiments within both sample groups at various intervals of fetal arterial oxygen content. The mean values are represented by dots, the standard deviations by bold lines and the range values by thin lines, respectively. 
Table 3.2. Mean changes of the variables from baseline condition to deepest hypoxemia (mesns $\pm \mathrm{SD}$ ). Statistical significance was tested by paired-comparisons t-test:

\begin{tabular}{lllll}
\hline & GROUP I $(\mathrm{n}=6)$ & GROUP II $(\mathrm{n}=4)$ \\
\hline variable & $\begin{array}{l}\text { baseline } \\
\text { condition }\end{array}$ & $\begin{array}{l}\text { deepest } \\
\text { hypoxemia }\end{array}$ & $\begin{array}{l}\text { p-value } \\
\text { condition }\end{array}$ & $\begin{array}{l}\text { deepest pypoxemia } \\
\text { hyalue }\end{array}$ \\
\hline
\end{tabular}

oxygen content (mM)

$2.91 \pm 0.32$

$0.81 \pm 0.25<0.0001$

$3.07 \pm 0.27 \quad 0.64 \pm 0.22<0.0001$

fetal heart rate (bpm)

$177 \pm 17$

$168 \pm 16$

n.s.

$180 \pm 20$

$177 \pm 16$ n.s.

mean

arterial pressure $(\mathrm{mmHg}) \quad 38.1 \pm 3.9 \quad 44.7 \pm 3.0 \quad<0.02 \quad 39.6 \pm 6.6 \quad 45.5 \pm 7.1<0.005$

umbilical artery $\mathrm{PI}$

$0.93 \pm 0.14 \quad 0.96 \pm 0.14$ na.s.

femoral antery $\mathbb{P I}$

\section{femaral artery blood flow velocity wave forms}

femoral artery $P \mid$
oxygen content (mM)
plit
base excess (mM)

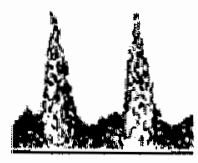

1.88
3.13
7.38
0.2

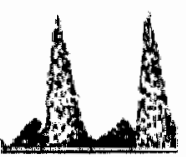

2.71

2.21

7.35

$-2.7$

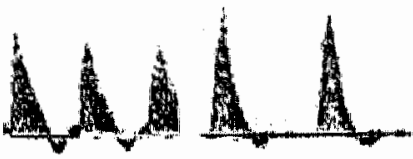

4.04

1.45

7.32

$-4.3$
6.52

0.79

7.24

Figure 3.3. Femoral artery blood flow velocity waveforms from one of the fetuses in group II and the corresponding values of femoral artery PI, oxygen content, $p H$ and base excess in the fetal abdominal aorta.

umbilical artery
blood flow velocity
waveforms

umbilical artery $P$ oxygen content ( $\mathrm{mM}$ ) $\mathrm{pH}$

base excess (mM)

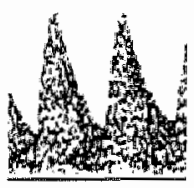
1.00
3.02
7.39
1.8

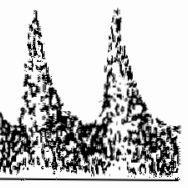

1.17

2.17

7.36

$-0.3$

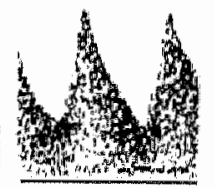

0.95

1.43

7.34

$-2.5$

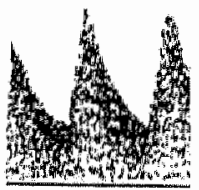

1.03

0.83

7.22

$-5.9$

Figure 3.4. Umbilical artery blood flow velocity waveforms from one of the fetuses in group II and the corresponding values of umbilical artery PI, oxygen content, $\mathrm{pH}$ and base excess in the fetal abdominal aorta. 
At the end of the experiment, release of the occluder resulted in a kaleidoscope of hemodynamic changes. Two fetal lambs died in utero, within and more than one hour after release of the occluder respectively: one fetus did not recover from hypoxemia and died because of cardiac failure (bradycardia, hypotension and deteriorating asphyxia); the other fetus died after a long period accompanied by sinusoidal fetal heart rate pattern. Four fetuses developed a prolonged tachycardia, whereas two others showed a saltatory heart rate pattem. In the remaining two fetal lambs, fetal heart rate did not change appreciably.

\section{DISCUSSION}

The purpose of this study was to evaluate the short-term response of the fetal umbilical and femoral artery pulsatility indices to various levels of fetal hypoxemia. The PI, being a close description of a blood flow velocity waveform, provides information on pulsatile blood flow at the site of measurement. Pulsatile blood flow in an artery is brought about by propulsive and impedant factors. Propulsion of blood flow is the result of heart rate, intraventricular volume and cardiac contractility. Impedance to blood flow is the result of resistance, compliance and inertia parameters (Ho et al., 1987). Although the individual contributions of these parameters are not completely elucidated, the PI virtually provides information on the relative extent of diastolic blood flow velocity. The continuum of reduction, absence or reversal of diastolic blood flow velocity results in an increase in the PI.

We observed that a gradual decrease in fetal oxygenation from 3.07 to 0.64 $\mathrm{mM}$ was associated with a concomitant increase in the femoral PI from 1.71 to 4.29 (table 3.2, figure 3.2). Figure 3.3 illustrates femoral artery blood flow velocity waveforms and values of the femoral PI at various levels of oxygen, observed in one of the fetuses in group II. In contrast with the femoral PI, the umbilical PI did not significantly change with $\left[\mathrm{O}_{2}\right]_{\mathrm{a}}$, as is depicted in the example in figure 4 . Since the changes in fetal heart rate and fetal mean arterial pressure in group I did not differ from those in group II, any discrepancy in the changes in umbilical PI and femoral PI with decreasing fetall oxygenation has to be explained by local differences in impedance to blood flow. Therefore, the results from the present study indicate that impedance to blood flow in the fetal femoral artery increases during hypoxemia, while that in the umbilical circulation does not significantly change. The umbilical circulation seems thus to play a minor role in the regulation of blood flow redistribution during fetal hypoxemia. This inference from the results corresponds with data from previous reports. 
Peeters et al. (1979) administered gas mixtures of nitrogen, oxygen and carbon dioxide to pregnant ewes and investigated the distribution of blood flow in a group of 12 fetal lambs at various levels of oxygen. Within this group, umbilical blood flow did not consistently change as a function of fetal $\left[\mathrm{O}_{2}\right]_{\mathrm{a}}$. Fetal hypoxemia induced by graded reductions in uterine blood flow did result in a decrease in umbilical blood flow, however, only if associated with fetal bradycardia (Cohn et al., 1985; Yaffe et al., 1987). In order to prevent substantial changes in fetal heart rate, the decline in fetal $\left[\mathrm{O}_{2}\right]_{\text {a }}$ was induced very gradually (van Huisseling et al., 1989). Major changes in fetal heart rate and umbilical PI were rare in the $\left[\mathrm{O}_{2}\right]_{\mathrm{a}}$ range between 3.0 and $1.0 \mathrm{mM}$. A tendency toward spontaneous fetal heart rate decelerations was observed at $\left[\mathrm{O}_{2}\right]_{a}$ levels below $1.0 \mathrm{mM}$. When fetal $\left[\mathrm{O}_{2}\right]_{\mathrm{a}}$ has decreased to below $2.0 \mathrm{mM}$, fetal oxygen demand exceeds oxygen availability (Peeters et al., 1979). Noninvasive methods for fetal surveillance with a high diagnostic value for fetal oxygenation between 2.0 and $1.0 \mathrm{mM}$ have not yet been reported. Apart from the present data, previous reports from studies in the human fetus also indicate that the umbilical artery blood flow velocity waveform is a relatively weak parameter in predicting or diagnosing fetal distress (Trudinger et al., 1986; Ferrazzi et al., 1988). Therefore, we also focused on changes in the femoral artery blood flow velocity waveform. To our knowledge, all studies on fetal cardiovascular responses and blood flow distribution during hypoxemia demonstrate an inverse relationship between fetal oxygenation and vascular resistance in skeletal muscle and skin in the $\left[\mathrm{O}_{2}\right]_{\mathrm{a}}$ range between 3.0 and $1.0 \mathrm{mM}$ (Peeters et al., 1979; Cohn et al., 1985; Yaffe et al., 1987). It was expected that this elevated femoral vascular resistance on the basis of chemoreceptor activation, would produce a change in femoral artery blood flow velocity waveforms. The results from the present study indeed demonstrate an inverse relationship between $\left[\mathrm{O}_{2}\right]_{\mathrm{a}}$ and femoral PI. The observed inverse covariance between umbilical PI and fetal heart rate is in accordance with studies in the human (Mulders $e t$ al., 1986) and in the ovine fetus (van Huisseling et al., 1989).

In clinical obstetrics, attention has also been paid to the possible diagnostic value of Doppler investigation of the cerebral circulation (Wladimiroff $e t a l$, 1986). Probably as a result of the brain-sparing effect, a relative decrease in cerebral artery Pulsatility Index seems to correlate with fetal hypoxia. Experimental Doppler investigation of the carotid artery in fetal sheep is of little value because of anatomical differences, whereas investigation of the downstream ovine fetal cerebral circulation is technically laborious or prone to fail. The pattern of cardiac output distribution during hypoxemia supports our hypothesis 
that simultaneous measurement of blood flow velocity waveforms in various fetal vessels may result in a new noninvasive method for the detection of mild to moderate fetal hypoxemia.

The results of this study do not deny the possible clinical value of the umbilical artery blood flow velocity waveform analysis in the detection of fetal compromise, since abnormal waveforms may result from morphological or functional changes in the umbilical circulation (Giles et al., 1985), but it also follows from the present data that severe hypoxemia can exist, without any change in the umbilical artery blood flow velocity waveform.

It is concluded from this study that gradual reductions in uteroplacental blood flow can result in a condition with severe fetal hypoxemia and normal fetal heart rate. This condition is associated with increased femoral artery Pulsatility Index. In contrast, umbilical artery Pulsatility Index does not significantly change during short-term fetal hypoxemia, but in correspondence with the normal human fetus, covaries inversely with fetal heart rate. 


\section{CHAPTER 4}

\section{The effect of maternal hypoxemia on the umbilical and femoral artery blood flow velocity waveforms and the relationship with mean arterial pressure in fetal sheep}

G.J.J.M. Muijsers, H. van Huisseling and T.H.M. Hasaart

Submitted for publication

\section{ABSTRACT}

The relationship between the ratio femoral PI/umbilical PI and arterial oxygen content was examined in eight fetal sheep at various stages of hypoxemia. This relationship was characterized by an inconsistent increase of the ratio femoral PI/umbilical PI at oxygen content levels beneath $1.0 \mathrm{mM}$. The correlation coefficient of the linear regression between both variables was only -0.54 , indicating that prediction of fetal oxygenation on the basis of Doppler waveform measurements in peripheral fetal arteries might be invalid. The average correlation coefficient of the linear regressions between the femoral PI and fetal mean arterial pressure was $0.87 \pm 0.15$ (SD), whereas the average correlation between the umbilical PI and fetal mean arterial pressure was $0.04 \pm 0.61$. These data suggest that the degree of change in fetal mean arterial pressure considerably depends on the degree of change in vascular resistance in the fetal carcass. 


\section{INTRODUCTION}

Currently, fetal Doppler blood flow velocimetry is recommended as a tool for clinical management policy (Trudinger \& Cook, 1990). However, the indications for clinical Doppler measurements are not well standardized and the conclusions to be drawn from the results are not well specified (Neilson, 1987; Redman, 1989). As randomized controlled trials might provide clarity on the clinical usefulness, basic research might provide more insight in the biological significance of Doppler blood flow velocimetry in perinatal medicine.

It has been shown in sheep fetuses that acute fetal hypoxemia is associated with a series of cardiovascular adaptation mechanisms, such as bradycardia, hypertension and a redistribution of blood flow, resulting from myocardial, cerebral and adrenal vasodilatation, and from intestinal, pulmonary and musculocutaneous vasoconstriction (Peeters et al., 1979). The response of the umbilical circulation seems, more than that of other vascular beds, to be related with factors such as mechanism, duration and severeness of hypoxemia (Rudolph, 1976). One of the effects of vasoconstriction in skeletal muscle and skin is an increase in impedance to blood flow in the hindlimb circulation. Thus, fetal hypoxemia may indirectly result in an increase in impedance to blood flow in the fetal femoral artery. The Pulsatility Index (PI), as determined by Doppler ultrasound, is assumed to reflect impedance at the site of measurement (McGallum et al., 1978). In a previous study it has been shown that the PI of the femoral artery blood flow velocity waveform (femoral PI) increased in each of four fetal lambs, whereas the PI of the umbilical artery blood flow velocity waveform (umbilical PI) in a group of six fetal lambs did not change in response to progressively reduced uterine blood flow (Muijsers et al., 1990). As a consequence, the femoral PI might be used as a tool for noninvasive estimation of fetal oxygenation. Unfortunately however, the value of a waveform index also depends on variables such as heart rate (Mulders et al., 1986; van Huisseling et al., 1989). In order to dispose of a parameter possibly less sensitive to variations in heart rate and more sensitive to variations in fetal blood flow distribution, we also determined the umbilical PI. This study was designed to examine the hypothesis that, in normally grown fetal sheep, the ratio femoral PI/umbilical PI correlates with fetal oxygen content to such a degree that this ratio may be used for noninvasive estimation of fetal oxygenation. 


\section{MATERIALS AND METHODS}

Surgery. The experiments were performed in eight ewes of the Dutch Texel breed between the 110th and 125th day of gestation (term 147 days). Surgical instrumentation was performed under aseptic conditions and general anesthesia. Anesthesia was induced with pentobarbital and maintained with $1 \%$ halothane in a 2:1 mixture of nitrous oxide and oxygen. Prior to surgery, the ewes received $1 \mathrm{~g}$ of ampicillin intravenously. After opening the abdomen through a paramedian incision, the uterus was incised over the fetal pelvis and the fetal legs were exteriorized. A polyvinyl catheter (outer diameter 1.6/inner diameter $0.8 \mathrm{~mm}$ ) was inserted into one of the femoral arteries and advanced into the abdominal aorta. Then, a $20 \mathrm{MHz}$ pulsed Doppler device, fixed in a polyvinyl cuff, was placed around the contralateral femoral artery. Another polyvinyl catheter, for registration of intra-amniotic pressure, was secured to the fetal skin. The catheters and Doppler lead were exteriorized through a skin incision in the ewe's flank. The guidelines for the care and use of animals approved by the local Animal Medical Ethics Committee were followed.

Measurements. Fetal arterial pressure and intra-amniotic pressure were determined with the zero point at the level of the ewe's spine. Fetal mean arterial pressure was defined as the pressure difference between the fetal aorta and the amniotic cavity. Fetal heart rate registration was derived from the arterial pressure waveform. The pressure- and heart rate signals were fed into amplifiers (Hewlett Packard 8800 Series, Hewlett Packard, Andover, Massachusetts, USA), displayed on monitor, recorded on strip chart recorder, and stored on magnetic tape. Fetal heart rate and fetal mean arterial pressure, averaged by computer from ten values over a 10-seconds period, were determined simultaneously with the blood flow velocity waveforms. Fetal blood samples were taken immediately after registration of the cardiovascular parameters and tested for hemoglobin concentration and oxygen saturation (OSM2 Hemoximeter, Radiometer, Copenhagen) in order to calculate oxygen content. $\mathrm{PCO}_{2}, \mathrm{pH}$ and base excess were determined by blood gas analyzer at $39^{\circ}$ Celsius (ABL, Radiometer, Copenhagen, Denmark). Blood flow velocity waveforms in the femoral artery were obtained by means of the implanted $20 \mathrm{MHz}$ pulsed Doppler transducer (545C-4 Directional Pulsed Doppler Flowmeter, Bioengineering, University of Iowa, lowa City, USA). Umbilical artery flow velocity waveforms were obtained using a $4 \mathrm{MHz}$ continuous wave Doppler transducer (Delalande Electronique, $300 \mathrm{~Hz}$ high-pass filter, Courbevoie, France) which was insonated from the abdominal skin of the standing ewe. The origin of the received signals was verified by localization of the umbilical cord using a two-dimensional linear array scanner (Scanner 400, Sanyo, Japan), by compari- 
son of fetal heart rate derived from the pressure and velocity waveforms respectively, and by the presence of reversely directed venous umbilical blood flow in the Doppler registration. The frequency shifted, analog signals, were stored in quadrature on audiotape and processed by a spectrum analyser (Doptek Lid., Chicester, England). The frequency-time spectra, as generated by the spectrum analyser, were quantified off-line by means of the PI. In our experiments, this parameter is defined as the difference between the maximum and minimum frequency shift divided by the mean frequency shift of the maximum envelope curve throughout the cardiac cycle (Gosling et al., 1971; Ruissen et al., 1987a).

Experiments. After surgical instrumentation, the animals were allowed to recover for at least three days. To produce fetal hypoxemia, the maternal head was enclosed in a large plastic bag, which was insufflated with nitrogen, varying amounts of oxygen, and carbon dioxide, in order to maintain the fetal $\mathrm{PaCO}_{2}$ within the normal range. The flow of carbon dioxide was maintained on approximately $250 \mathrm{ml} / \mathrm{min}(1-3 \%)$, while the flow of nitrogen was maintained on $10.000 \mathrm{ml} / \mathrm{min}$. Only the oxygen flow was altered and varied between 900 and $2.700 \mathrm{ml} / \mathrm{min}(8-21 \%)$. Baseline values $(t=0)$ were measured after 30 minutes of maternal adaptation to the plastic bag, while the oxygen concentration was kept on 21 vol\% using an oxygen flow meter (Oxycom 100D, Draegerwerk $A G$, Luebeck, West Germany). Subsequently, the oxygen flow was acutely reduced to 12 vol\%. Blood samples were taken in order to adjust fetal oxygenation between 1.0 and $2.0 \mathrm{mM}$. After approximately 60 minutes $(t=60)$, all variables were measured at stable readings. Then, oxygen flow was reduced to $8-9 \mathrm{vol} \%$, while small adjustments were made to maintain fetal oxygenation beneath $1.0 \mathrm{mM}$. At $t=120 \mathrm{~min}$, all variables were measured simultaneously again. Finally, the ewe was provided with gas of room air composition during 60 minutes and at $t=180 \mathrm{~min}$, all variable values were registered after which the experiment was terminated.

Calculations and data analysis. Five consecutive femoral and umbilical artery blood flow velocity waveforms, simultaneously registered on tape during stable readings, were anallysed for PI values. Before acceptance, the coefficient of variation of the mean values should be less than 10 percent. The mean variable values measured at 60,120 and $180 \mathrm{~min}$ were compared with those at baseline condition using Wilcoxon's matched pairs signed rank test. The Bonferroni inequality was used to adjust for multiple comparisons (Wallenstein et al., 1980). A regression line and prediction interval was calculated for for description of the relationship between the ratio femoral PI/umbilical PI and oxygen content (Johnson, 1984). In addition, regression lines were calculated to provide information on both the individual and sample group trends. 


\section{RESULTS}

Acute reduction of matemally inspired oxygen resulted in a transient bradycardia in almost all fetuses. After approximately 10 minutes, fetal heart rate returned to normal levels. Occasionally, shortlasting decelerations of less than 30 seconds varied with traces with normal fetal heart rate, especially at fetal oxygen content levels beneath $1 \mathrm{mM}$.

Table 4.1 lists the mean values $( \pm S D$ ) of all variables at $t=0,60,120$ and 180 minutes, by which $t=0$ min represents baseline condition, $t=60 \mathrm{~min}$ a condition in which oxygen content varies between 1.0 and $2.0 \mathrm{mM}, t=120$ min a condition in which oxygen content is below $1.0 \mathrm{mM}$, and $t=180$ min the condition after 60 minutes recovery from hypoxemia. $\mathrm{PaCO}_{2}, \mathrm{pH}$ and base excess could only be determined in 5 fetuses and not subjected to statistical analysis. Fetal oxygen content was significantly lower at all points of time when compared with baseline condition. Fetal heart rate and umbilical PI did not significantly change, although in seven fetuses, fetal heart rate at $t=180$ min exceeded the

Table 4.1. Mean values $( \pm S D)$ in 8 fetal sheep at $t=0$ min (baseline values), $t=60$ min $(1.0$ $\mathrm{mM}<$ oxygen content $<2.0 \mathrm{mM}$ ), $\mathrm{t}=120$ min (oxygen content $<1.0 \mathrm{mM}$ ) and $\mathrm{t}=180 \mathrm{~min}$ (recovery values). $\mathrm{PaCO}_{2}, \mathrm{pH}$ and base excess were determined in only 5 fetuses and not analysed for statistical significance. $\left.{ }^{*}\right)=p<0.05$ when compared with baseline condition using Wilcoxon's signed rank test and the Bonferroni inequality for multiple comparisons.

\begin{tabular}{lllll} 
variable & $t=0 \mathrm{~min}$ & $\mathrm{t}=60 \mathrm{~min}$ & $\mathrm{t}=120 \mathrm{~min}$ & $\mathrm{t}=180 \mathrm{~min}$ \\
\hline oxygen content $(\mathrm{mM})$ & $2.93 \pm 0.39$ & $1.52 \pm 0.30^{*}$ & $0.65 \pm 0.16 *$ & $2.29 \pm 0.56 *$ \\
$\mathrm{PaCO}(\mathrm{mmHg})$ & $44.9 \pm 4.5$ & $45.2 \pm 3.3$ & $42.8 \pm 5.0$ & $43.5 \pm 4.5$ \\
$\mathrm{pH}$ & $7.35 \pm 0.03$ & $7.33 \pm 0.04$ & $7.28 \pm 0.06$ & $7.31 \pm 0.05$ \\
base excess (mM) & $-0.2 \pm 1.4$ & $-1.9 \pm 2.6$ & $-7.0 \pm 1.2$ & $-3.2 \pm 3.8$ \\
fetal heart rate (bpm) & $174 \pm 19$ & $175 \pm 18$ & $172 \pm 23$ & $192 \pm 23$ \\
arterial pressure (mmHg) & $35.6 \pm 4.7$ & $36.9 \pm 4.6$ & $38.6 \pm 4.6$ & $36.5 \pm 6.7$ \\
femoral artery PI & $1.96 \pm 0.48$ & $2.36 \pm 0.34 *$ & $3.43 \pm 1.00 *$ & $1.85 \pm 0.39$ \\
umbilical artery PI & $1.00 \pm 0.16$ & $0.98 \pm 0.16$ & $1.00 \pm 0.15$ & $0.95 \pm 0.17$ \\
femoral PI/umbilical PI & $1.95 \pm 0.33$ & $2.44 \pm 0.34 *$ & $3.47 \pm 1.11 *$ & $2.02 \pm 0.69$
\end{tabular}



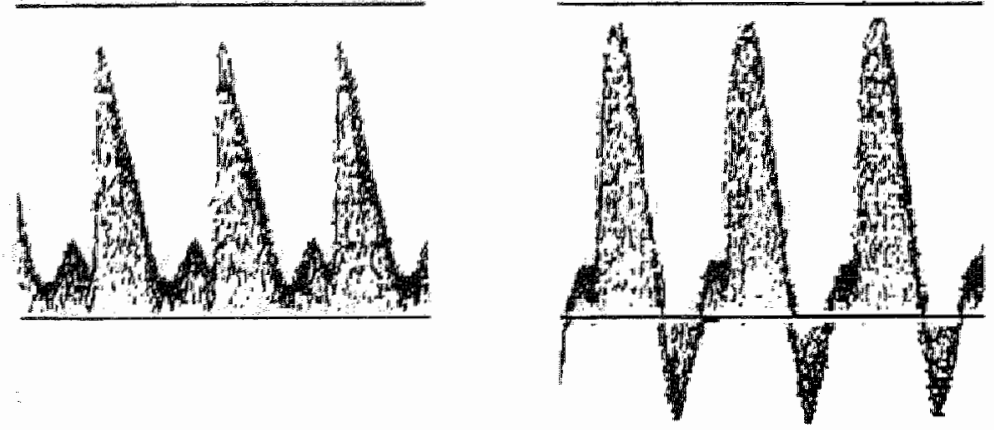

F1gure 4.1. Femoral artery blood flow velocity waveforms from one fetus at baseline condition (left panel) and during severe hypoxemia (right panel).

value at $\mathrm{t}=0$ min. Fetal mean arterial pressure showed an upward trend with decreasing oxygenation. However, these changes were not significant, mainly because of multiple comparisons. Femoral PI and the ratio femoral PI/umbilical PI were significantly higher at $t=60 \mathrm{~min}$ and $t=120 \mathrm{~min}$.

Figure 4.1 depicts the femoral artery blood flow velocity waveforms and concurrent PI values from one fetus at baseline condition and during severe hypoxemia.

Figure 4.2, upper panel,shows the linear regression line fitted to the data of oxygen content and the ratio femoral PI/umbilical PI. The curved lines express the upper and lower limits of the $95 \%$ confidence interval with regard to prediction of the ratio femoral PI/umbilical PI on the basis of a random value of oxygen content. This prediction interval indicates that in this experiment, oxygen content values of both $0.5 \mathrm{mM}$ and $3.5 \mathrm{mM}$ could be associated with ratio femoral $\mathrm{PI} / \mathrm{umbilical}$ PI values varying between 1.5 and 3.5 , respectively. As shown in figure 4.2, middle and lower panels, the relationship between femoral PI or umbilical PI and oxygen content does not provide better figures.

Determination of the intraindividual linear trend showed that the ratio femoral PI/umbilical PI increased in six and decreased in two fetuses with decreasing oxygen content (figure 4.3, upper panel). The same observations were made for the relationship between fetal mean arterial blood pressure and oxygen content. It was noted that an increase in the ratio femoral PI/umbilical PI was associated with an increase in mean arterial pressure and vice versa. It was hypothesized that increments in both femoral PI and mean arterial pressure mainly result from vasoconstriction in the fetal hindlimbs. Figure 4.3 , lower panel, shows the data points and the regression lines representing the relationship between the femoral PI and mean arterial pressure. The correlation coefficient of the regression between both variables varied between 0.56 and 1.00 . 
fremaro: antery Pl/umbilico ortery Pl

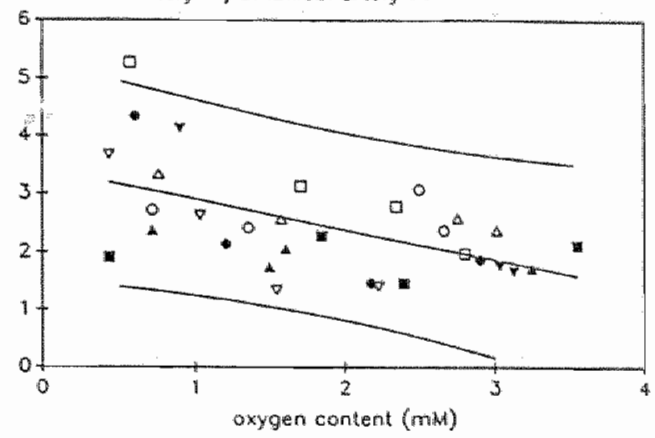

femorol ortery 8
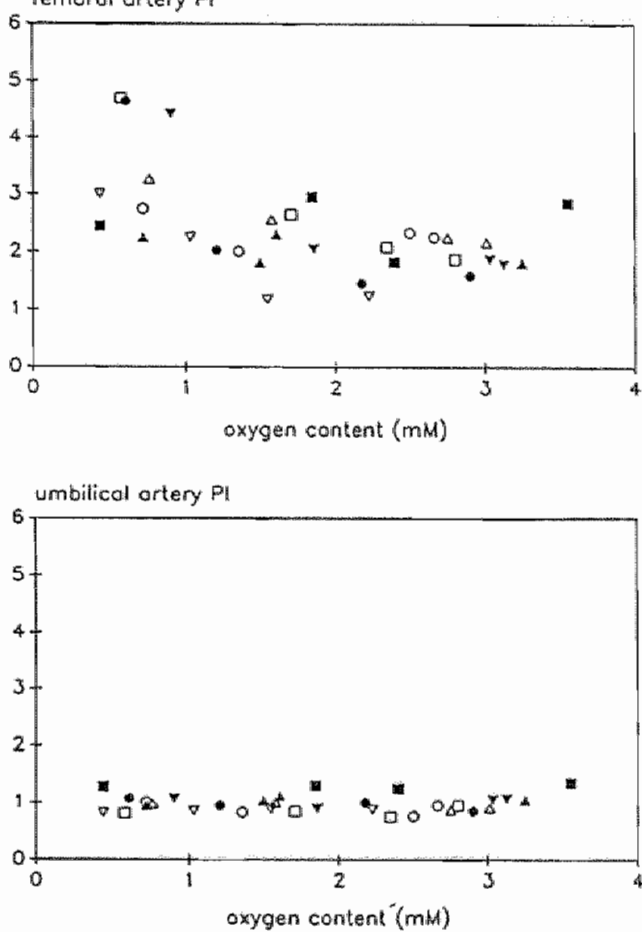

Figure 4.2. Upper panel: data points, regression line and prediction interval representing the relationship between the ratio femoral PI/umbilical PI and oxygen content as determined in 8 chronically-instrumented fetal sheep at 113-128 days' gestation. Middle panel: data points representing the relationship between the femoral PI and oxygen content. Lower panel: data points representing the relationship between the umbilical PI and oxygen content. 

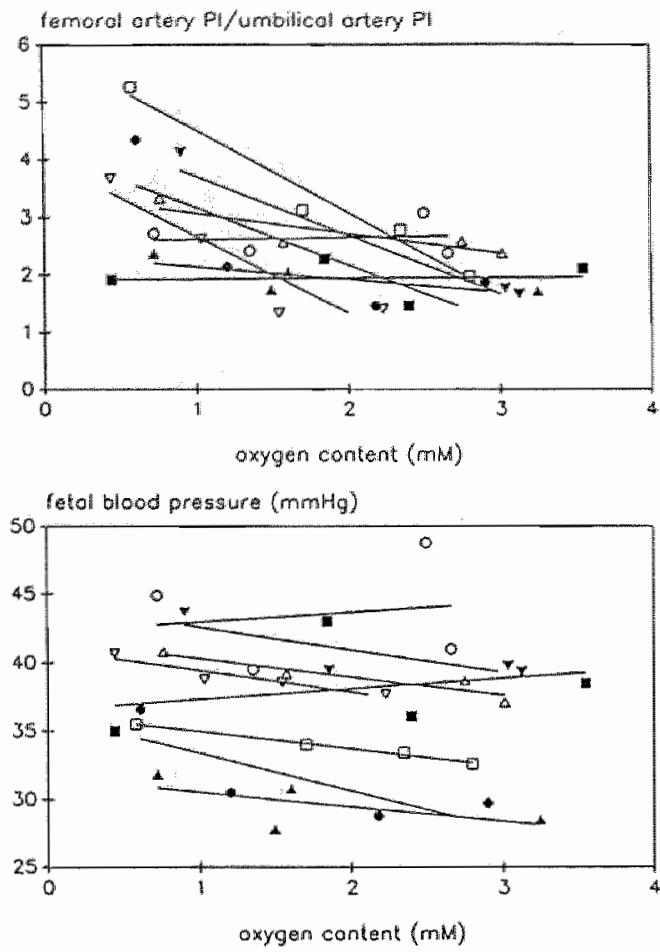

femoral artery

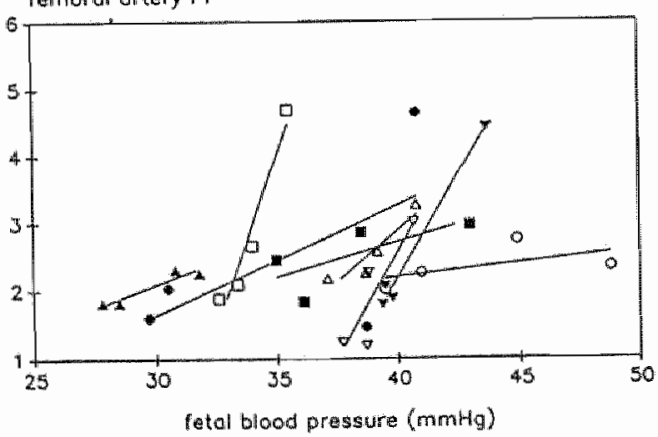

Figure 4.3. Upper panel: data points and regression lines representing the relationship between the ratio femoral PI/umbilical PI and oxygen content per fetus. Middle panel. data points and regression lines representing the relationship between fetal mean arterial pressure and oxygen content per fetus. Lower panell: data points representing the relationship between the femoral PI and fetal mean arterial pressure at various stages of hypoxemia. 
The average correlation coefficient was $0.87 \pm 0.15(\mathrm{SD})$. In contrast, the average correlation coefficient of the regressions between the umbilical PI and fetal mean arterial pressure was only $0.04 \pm 0.61$.

\section{DISCUSSION}

Methods of fetal surveillance are widely used in order to prevent morbidity and mortality in the perinatal period and to prevent the development of physical, mental or behavioural disorders during childhood. Methods as intermittent fetal heart rate auscultation and continuous fetal heart rate registration, are based on the emergence of typical changes in the fetal heart rate and/or fetal heart rate pattern, frequently observed in relation to imminent fetal hypoxia. Another feature of hemodynamics during hypoxia is the redistribution of blood flow, based on tissue-dependent changes in impedance to blood flow. These changes might be studied using noninvasive Doppler methods.

The reason that we studied the ratio femoral PI/umbilical PI as a measure of fetal blood flow redistribution was threefold. Firstly, the PI varies with fetal heart rate. It was presumed that the ratio of two synchronous measurements would provide a measure of blood flow distribution, relative insensitive to fetal heart rate variability. Secondly, instrumentation of the femoral artery is rather simple and it was presumed that hypoxemia would be associated with unambiguous hemodynamic changes in this blood vessel (Peeters et al, 1979). In contrast, the umbilical artery was regarded as a reference vessel, selected because of general interest and ease of measurement, and not because of presumed increments in PI values. In this respect, the role of the umbilical PI in this study considerably differs from that in other studies, where the umbilical PI is invariably supposed to be increased in relation with hypoxemia. Thirdly, experimental validation of Doppler flow measurements in for example the carotid artery might lead to ambiguous conclusions on the basis of large anatomical differences between the human and ovine cerebral and cranial circulations (Makowski et al., 1968).

As shown in figure 2, both the femoral PI and the ratio femoral PI/umbilical PI poorly correlate with fetal oxygenation, but this does not imply that Doppler waveform indices cannot be used for noninvasive assessment of fetal oxygenation. Against this study might be objected that it concerns a small sample group of non-human fetuses at various gestational ages with apparently normal fetoplacental development, in which oxygenation and possibly other variables were acutely changed. However, it also could be argued that assessment of fetal oxygenation using Doppler velocimetry will be invalid. Firstly, femoral PI 
values in some fetuses during hypoxemia were lower than those of others during normoxemia, while the range in gestational age was only 2 weeks. This suggest a wide interfetal variation of possible femoral PI values during normoxemia. Secondly, the femoral PI strongly correlated with mean arterial pressure in each of the studied fetuses. As observed in this study, hypoxemia might be parallelled by inconsistent changes in mean arterial pressure, suggesting that hypoxemia is not necessarily associated with hypertension. Since all fetuses were still alive on the next day, it is concluded the fetal lamb can withstand severe hypoxia for several hours without the emergence of overwhelming sympathetic reactions. However, the reasons of the inconsistent changes in fetal blood pressure in this study remain not well understood. Absence of fetal hypertension was not particularly associated with gross changes in fetal heart rate, aberrant blood gas values or deeper asphyxiation.

The availability of cordocentesis has made it possible now to correlate Doppler waveform indices with fetal oxygenation in thuman pregnancy. Vyas et al. (1989) found a linear correlation of -0.66 between renal artery Pulsatility Index and umbilical venous $\mathrm{PaO}_{2}$ in $\mathrm{SGA}$ fetuses. This correlation suggests that the human fetus reacts to hypoxemia with a corresponding pattern of blood flow redistribution as the sheep fetus. This correlation however appears relatively poor for reliable, noninvasive prediction of fetal oxygenation and therefore it seems that renal artery velocimetry is inappropriate as a test for fetal surveillance.

Bilardo et al. (1990) examined the ability of Doppler measurements in the fetal aorta, common carotid artery and umbilical artery to predict fetal oxygenation, also determined by cordocentesis. They found that a mathematical index of aortic mean blood flow velocity and common carotid artery Pulsatility Index (Ao-CC index) was the best predictor for intrauterine asphyxia. This finding also corresponds with the concept of fetal blood flow redistribution in response to hypoxia. However, the possibility of clinical applicability of these Doppler measurements as a method of fetal surveillance remains questionable too, since the linear correlation between the $\mathrm{Ao}-\mathrm{CC}$ index and a measure of intrauterine asphyxia was only -0.67 .

It is concluded from this study that the femoral PI increases in response to a decrease in fetal oxygen content. The relatively poor correlation between both variables however, does not allow speculations on applicability of Doppler velocimetry as a noninvasive test for assessment of fetal oxygenation. In each of the eight fetal lambs studied in this experiment, the femoral PI positively correlated with mean arterial pressure, regardless of the induced change in fetal oxygenation. We suggest that increments in the femoral PI and mean arterial pressure in this study resulted from the same mechanism, i.e. vasoconstriction 
of the precapillary sphincters in the fetal carcass. We speculate that measurement of blood flow velocity waveforms in various fetal vessels may provide noninvasive information on (changes in) the pattern of blood flow distribution. Whether fetal waveform indices can provide noninvasive information on fetal blood pressure, should be examined in experiments in which blood pressure is selectively changed. 



\section{CHAPTER 5}

\section{The response of the umbilical artery}

\section{Pulsatility Index in fetal sheep to acute and prolonged hypoxemia and acidemia induced by embolization of the uteroplacental microcirculation}

G.J.J.M. Muijsers, T.H.M. Hasaart, H. van Huisseling, J. de Haan

Journal of Developmental Physiology, in press

\section{ABSTRACT}

In eight chronically-instrumented sheep, embolization of the uterine microcirculation was performed to evaluate the response of the pulsatility index of the umbilical artery blood flow velocity waveform (umbilical PI) to prolonged fetal hypoxemia and acidemia. From four days after surgery onwards, fetal arteriall oxygen content $\left(\left[\mathrm{O}_{2}\right]\right.$ a) was progressively reduced by administration of microspheres into the uterine circulation. Measurements included fetal $\left[\mathrm{O}_{2}\right] \mathrm{a}, \mathrm{PaO}_{2}$, $\mathrm{PaCO}_{2}, \mathrm{pH}$, base excess, heart rate, blood pressure and umbilical PI. Fetal survival varied between less than 2 and less than 8 days, while mean fetal survival was less than 4 days. From baseline condition to the last evaluation preceding the diagnosis of fetal death, $\left[\mathrm{O}_{2}\right]$ a decreased from $3.10 \pm 0.36$ to 0.87 $\pm 0.27 \mathrm{mM}, \mathrm{pH}$ decreased from $7.36 \pm 0.03$ to $7.22 \pm 0.08$, base excess decreased from $-0.3 \pm 1.5$ to $-7.3 \pm 3.2$ and blood pressure increased from 35.0 \pm 7.1 to $40.7 \pm 8.7$ (means \pm SD). The umbilical PI (1.05 \pm 0.19 at baseline condition) did not significantly change ( $1.08 \pm 0.12$ prior to fetal death). It is 
concluded that a condition of prolonged hypoxemia and acidemia in fetal sheep, induced by repeated embolizations of the uterine circulation, is not associated with consistent changes in the umbilicall PI.

\section{INTRODUCTION}

From clinical studies, it is concluded that indices of umbilical artery blood flow velocity waveforms can predict the emergence of perinatal distress (Reuwer et al., 1987) or compromise (Trudinger et al., 1987). The Pulsatility Index of the umbilical artery blood flow velocity waveform (umbilical PI) is assumed to reflect impedance to umbilical blood flow at the site of measurement (Gosling et al., 1971; McGallum et al., 1978). An increase in umbilical PI is ascribed to increased impedance to umbilical blood flow and is biophysically mainly characterized by the continuum of reduced, absent or reversed blood flow velocity during diastole. It has been demonstrated in fetal sheep that changes in the umbilical artery blood flow velocity waveform are associated with changes in umbilical blood flow and calculated umbilical vascular resistance (Trudinger et al., 1987b). Thus, fetal distress or compromise would be associated with hemodynamic changes in the umbilical circulation. Ideally however, a quantitive parameter such as the umbilical PI should correlate with a well-defined and generally accepted standard of intrinsic fetal well-being. In this study, fetal arterial oxygen availability as a prerequisite for aerobic metabolism, was used as a quantitative reference for fetal well-being. For valid prediction of individual fetal oxygen availability, the umbilical PI should correlate strongly with fetal $\left[\mathrm{O}_{2}\right] \mathrm{a}$, regardless of the mechanism of hypoxemia (Colton, 1974). It is beyond dispute that a prolonged decrease in umbilical blood flow, as induced by embolization of the umbilical circulation, can result in fetal hypoxemia. The possibility, however, of an opposite mechanism, that is that prolonged fetal hypoxemia results in a decrease in umbilical blood flow and increase in umbilical vascular resistance, is more controversial (Clapp et al., 1980; Parer, 1980; Bocking et al., 1988; Scheffen et al., 1989). Since embolization of the umbilical circulation does not only affect umbilical blood flow, but also placental vascular compliance, this model does not provide full insight into the relationship between fetal oxygenation and umbilical artery blood flow velocity waveforms. Various clinical conditions, such as maternal hypertension, retroplacental hemorrhage, multiple placental infarction, post-term pregnancy and prolonged labor, which are often associated with acute and/or prolonged fetal hypoxemia, seem to correspond better with the model achieved by embolization of the uterine circulation. In order to evaluate the response of the umbilical PI in fetal 
sheep to prolonged hypoxemia and acidemia, without immediate effect on fetal placental vascular compliance, moderate and severe fetal hypoxemia were induced by progressive embolizations of the uterine microcirculation.

\section{MATERIAL AND METHODS}

Surgery. The experiments were carried out in 8 ewes of the Dutch Texel breed. Surgical instrumentation was performed under aseptic conditions and general anesthesia between the 106th and 125th day of gestation. Anesthesia was induced with pentobarbital and maintained with $1 \%$ halothane in a $2: 1$ mixture of nitrous oxide and oxygen. Prior to surgery, the ewes received $1 \mathrm{~g}$ of ampicillin intravenously. The uterus was incised over the fetal pelvis and the fetal legs were exteriorized. A polyvinyl catheter was inserted into one of the femoral arteries and advanced into the abdominal aorta. Another polyvinyl catheter for measurement of intra-amniotic pressure was secured to the fetal skin. The uterus was closed and polyvinyl catheters were inserted into branches of the right and left uterine circulation and advanced into the main uterine arteries (Creasy et al., 1972). The catheters were exteriorized through a skin incision in the ewe's flank. The guidelines for the care and use of the animals approved by the local Animal Medical Ethics Committee were followed.

Measurements. Fetal aortic pressure and intra-amniotic pressure were determined with the zero point at the level of the ewe's spine. Fetal blood pressure was defined as the pressure difference between fetal aorta and amniotic cavity. Fetal heart rate registration was derived from the arterial pressure pulse. All signals were fed into amplifiers (Hewlett Packard 8800 Series, Andover, Massachusetts, USA), displayed on monitor, recorded on eightchannel strip chart recorder, and stored on magnetic tape. Fetal heart rate and fetal blood pressure, averaged by computer from 10 values over a 10 -seconds period, were determined simultaneously with the blood flow velocity waveforms. Fetal heart rate, derived form the aortic pressure waveform, was compared with fetal heart rate derived from the umbilical artery blood flow velocity waveform. Fetal blood samples were taken in duplicate immediately after registration of the cardiovascular parameters and tested for fetal arterial hemoglobin concentration and oxygen saturation (OSM2 Hemoximeter, Radiometer, Copenhagen) in order to calculate fetal arterial oxygen content $\left(\left[\mathrm{O}_{2}\right] \mathrm{a}\right)$. Furthermore, the samples were tested for arterial $\mathrm{pH}, \mathrm{PaO}_{2}$ and $\mathrm{PaCO}_{2}$ at 39 degrees Celcius (ABL, Radiometer, Copenhagen, Denmark), including automatic calculation of base excess. Only if both samples provided virtually identical values, the first sample was used for data analysis. Blood flow velocity waveforms in the umbilical 
artery were obtained using a $4 \mathrm{MHz}$ continuous wave Doppler transducer (Delalande Electronique, $300 \mathrm{~Hz}$ high-pass filter (lowest level), Courbevoie, France), which was handled from the abdominal skin of the standing ewe. The origin of the received signals was verified by localization of the umbilical cord using a two-dimensional ultrasound scanner (Scanner 400 , Sanyo, Japan), by comparison of fetal heart rate derived from pressure pulse and velocity pulse respectively, and by presence of oppositely directed venous umbilical blood flow within the Doppler sample volume. Blood flow velocity waveforms were only accepted for evaluation when fetal heart rate was stable for at least 5 minutes before and after the time point of registration. The frequency shifted signals were stored in quadrature on audiotape, processed by a spectrum analyzer (Doptek Ltd., Chicester, England) and quantified by means of the PI. The PI, as used in our experiments, is defined as the difference between the maximum and minimum frequency shift divided by the mean frequency shift of the maximum envelope curve throughout the cardiac cycle (Gosling et al., 1971; Ruissen et al., 1987a). Calculation of the PI values was performed off-line by drawing the maximum envelope curves of the frequency-time spectra with a handguided light-pen (Ruissen et al., 1987a). The umbilical PI, as used in the the data analysis, was calculated as a mean value, averaged over 5 consecutive cardiac cycles. Umbilical PI values with a coefficient of variation of more than $10 \%$ were discarded from analysis.

Experiments. After surgical instrumentation, the animals were allowed to recover for 4 days. Baseline values of acid-base balance, $\left[\mathrm{O}_{2}\right] \mathrm{a}$, fetal heart rate, fetal blood pressure and umbilical PI were measured over a 60 -minutes period. Subsequently, embolization of the uterine microcirculation was performed by a bolus injection of $1 \mathrm{cc}$ sterilized mucopolysacharide particles ((Sephadex G-25, Pharmacia, Uppsala, Sweden) (1\% weight/volume suspended in $0.9 \%$ saline solution, particle size: \pm 20 micron, number of particles in $\left.1 \mathrm{cc:} \pm 1.5 \times 10^{6}\right)$ ) into the uterine artery that supplied the pregnant horn. This procedure was repeated until fetal [ $\mathrm{O}_{2}$ ] a had decreased to below $2.0 \mathrm{mM}$. After 10 and 120 minutes, fetal heart rate, fetal blood pressure, umbilical $\mathrm{PI},\left[\mathrm{O}_{2}\right] \mathrm{a}$, blood gases and $\mathrm{pH}$ were measured for evaluation of the acute and subacute responses, respectively. On subsequent days, all variables used for evaluation were measured without preceding embolization procedures. Thereafter, fetal $\left[\mathrm{O}_{2}\right] \mathrm{a}$ was reduced to below $1.5 \mathrm{mM}$ on the second day and to below $1.0 \mathrm{mM}$ on the third and following days of experimentation. No embolization procedures were performed if the actual values of fetal $\left[\mathrm{O}_{2}\right]$ a were lower than the intentional values. The catheter at the non-pregnant horn was used for embolization, if repetitive microsphere administrations failed to lower fetal $\left[\mathrm{O}_{2}\right] \mathrm{a}$. 
Data analysis. The values of the variables obtained at 10 and $120 \mathrm{~min}$ after reduction of fetal $\left[\mathrm{O}_{2}\right] \mathrm{a}$ on the first day of experimentation (acute and subacute measurements, respectively) and the values obtained on the day prior to the diagnosis of fetal death or premature delivery (chronic measurements) were subjected to nonparametric paired comparison with those obtained during baseline condition (Wilcoxon signed rank test, Bonferroni inequality for multiple analyses). Thus, it was possible to perform independent paired comparisons at three different conditions in relation to the emergence of fetal hypoxemia: acutely $(10 \mathrm{~min})$, subacutely $(120 \mathrm{~min})$ and chronically (on the day prior to the diagnosis of fetal death, which day could differ per fetus and thus hindered representative paired comparisons at 24-hours intervals).

\section{RESULTS}

The number of embolizations, needed to reduce fetal $\left[\mathrm{O}_{2}\right]$ a to below $2.0 \mathrm{mM}$ on the first day of experimentation varied between one and four. The maximum number of embolizations in one animal over the whole experiment amounted to eleven. Table 5.1 lists the values of fetal [ $\left.\mathrm{O}_{2}\right]$ a at baseline condition, 10 and 120 min after reduction of $\left[\mathrm{O}_{2}\right]$ a to below $2.0 \mathrm{mM}$ and on the subsequent days. The number of embolizations and the times of administration are indicated too.

Table 5.1. The values of fetal [O2]a (in $\mathrm{mM}$ ) in eight fetal sheep (I-VIII) and the number of uteroplacental bolus embolizations subsequently administered (represented by asterixes).

day 1

day 2 day 3 day 4 day 5 day 6 day 7 day 8

baseline $10 \mathrm{~min} 120 \mathrm{~min}$

\begin{tabular}{lllllllllll}
\hline I & $3.23^{* * * * *}$ & 1.53 & 1.49 & $1.55^{*}$ & $1.31^{*}$ & $1.17^{*}$ & & & & \\
II & $3.55^{* * * *}$ & 1.67 & 1.85 & $2.55^{* *}$ & $1.03^{*}$ & $1.13^{*}$ & $1.47^{*}$ & 0.83 & $1.17^{*}$ & 1.04 \\
III & $2.90^{* *}$ & 1.86 & 1.25 & 1.25 & 0.39 & 0.37 & & & & \\
IV & $3.39^{* * * *}$ & 1.87 & 1.63 & 1.43 & $1.25^{*}$ & 0.70 & & & & \\
V & $2.59^{* *}$ & 1.85 & $2.05^{*}$ & 1.19 & 1.06 & 0.72 & 0.70 & & \\
VI & $3.35^{* * *}$ & $2.09^{*}$ & 1.19 & 1.06 & 0.72 & 0.70 & & & \\
VII & $2.60^{* *}$ & 0.94 & 0.97 & 0.85 & $1.08^{*}$ & 0.80 & 0.87 & & \\
VIII & $3.18^{*}$ & 0.22 & 0.31 & 1.02 & & & & & &
\end{tabular}



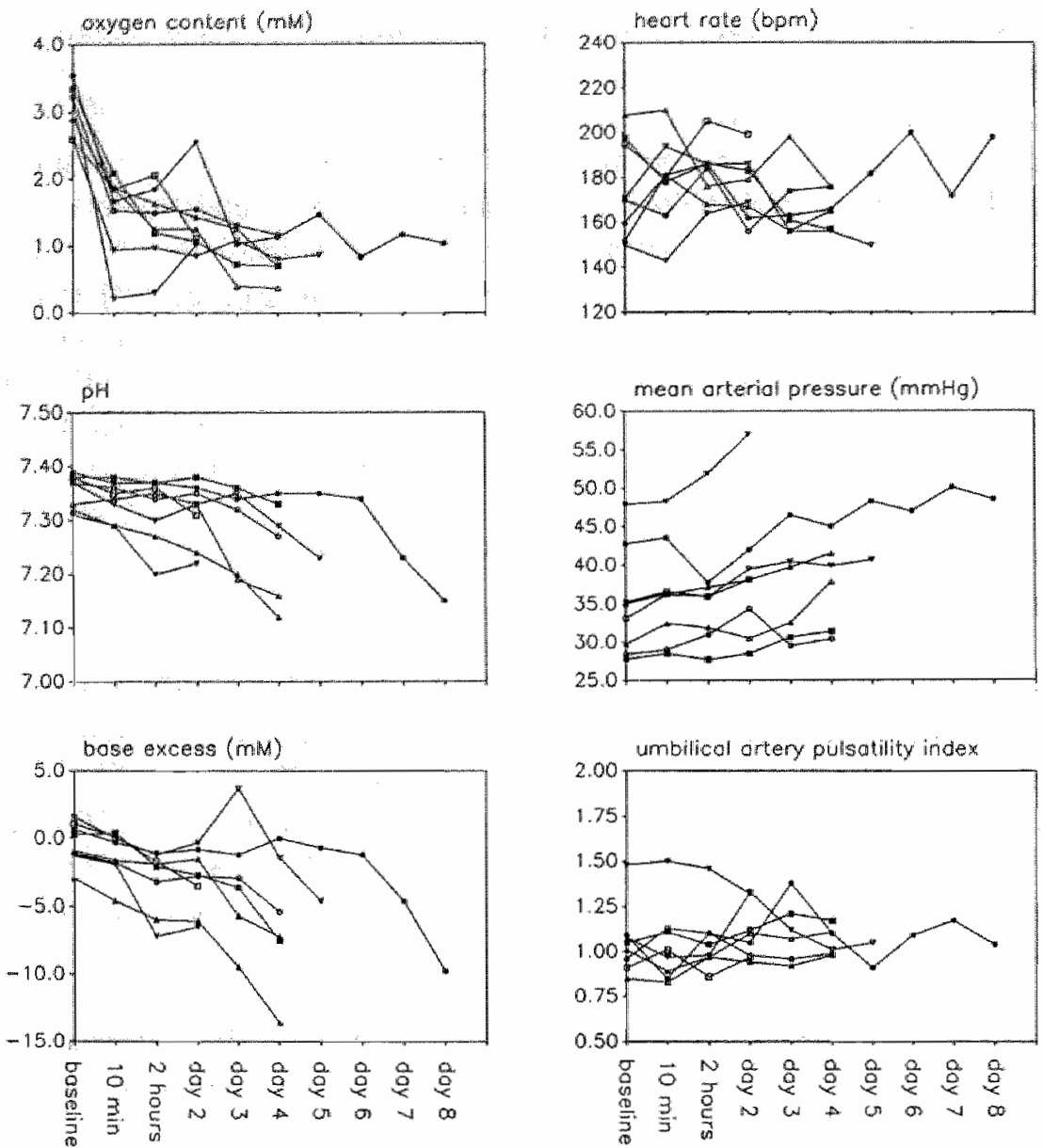

Figure 5.1. The acute and prolonged course of arterial oxygen content, $\mathrm{pH}$, base excess, heart rate, mean arterial pressure, and umbilical artery PI in eight fetal sheep following uteroplacental embolization.

Fetal survival varied between less than 2 and less than 8 days after embolization: one fetus survived for less than 2 days, four fetuses for less than 4 days, two fetuses for less than 5 days and one fetus for less than 8 days. Figure 5.1 illustrates the acute and prolonged course of fetal $\left[\mathrm{O}_{2}\right] \mathrm{a}, \mathrm{pH}$, base excess, heart rate, blood pressure and umbilical PI respectively. Administration of microspheres resulted in an acute decrease in fetal oxygenation. As follows from figure 5.1 (left upper panel) the fetal ability to recover from hypoxemia, induced by uteroplacental embolization, appeared to be poor. The time of deterioration of the fetal acid-base status varied per fetus. However, it is suggested that if 
Table 5.2. Mean values $( \pm \mathrm{SD})$ at baseline condition, 10 and 120 min after fetal $[\mathrm{O} 2]$ a had decreased to below $2.0 \mathrm{mM}$ on the first day of experimentation and on the last day preceding the diagnosis of fetal death or premature delivery $(n=8$ fetal sheep), $*$ p $<0.05$ when compared with baseline condition using Wilcoxon"s signed rank test and the Bonferroni inequality for multiple comparisons.

\begin{tabular}{|c|c|c|c|c|}
\hline & \multicolumn{3}{|l|}{ first day } & \multirow{2}{*}{$\frac{\text { last day }}{\text { baseline }}$} \\
\hline & baseline & $10 \mathrm{~min}$ & 120 min & \\
\hline$\left[\mathrm{O}_{2}\right] \mathrm{a}(\mathrm{mM})$ & $3.10 \pm 0.36$ & $1.50 \pm 0.62 *$ & $1.34 \pm 0.55 *$ & $0.87 \pm 0.27 *$ \\
\hline $\mathrm{PaO}_{2}(\mathrm{mmHg})$ & $19.7 \pm 2.6$ & $9.3 \pm 3.5 *$ & $8.7 \pm 3.1 *$ & $5.9 \pm 1.4 *$ \\
\hline $\mathrm{PaCO}_{2}(\mathrm{mmHg})$ & $47.1 \pm 1.4$ & $47.8 \pm 3.7$ & $46.7 \pm 6.0$ & $49.8 \pm 8.2$ \\
\hline $\mathrm{pH}$ & $7.36 \pm 0.03$ & $7.34 \pm 0.03$ & $7.32 \pm 0.06$ & $7.22 \pm 0.08 *$ \\
\hline base excess (mM) & $-0.3 \pm 1.5$ & $-1.2 \pm 1.7 *$ & $-3.1 \pm 2.3 *$ & $-7.3 \pm 3.2 *$ \\
\hline heart rate (bpm) & $176 \pm 22$ & $179 \pm 20$ & $182 \pm 13$ & $174 \pm 18$ \\
\hline blood pressure $(\mathrm{mmHg})$ & $35.0 \pm 7.1$ & $36.4 \pm 6.8 *$ & $36.1 \pm 7.2$ & $40.7 \pm 8.7$ \\
\hline umbilical artery PI & $1.05 \pm 0.19$ & $1.04 \pm 0.22$ & $1.06 \pm 0.18$ & $1.08 \pm 0.12$ \\
\hline
\end{tabular}

fetal $\left[\mathrm{O}_{2}\right]$ a falls below $1.5 \mathrm{mM}$, acidemia progressively increases. Figure 5.1 (right upper and lower panel) illustrates that heart rate and umbilical PI varied inconsistently within each fetus. Blood pressure however (figure 5.1, right middle panel), showed a consistent gradual increase during the follow-up period.

Table 5.2 lists the mean values and standard deviations of fetal $\left[\mathrm{O}_{2}\right] \mathrm{a}, \mathrm{PaO}_{2}$, $\mathrm{PaCO}_{2}, \mathrm{pH}$, base excess, heart rate, blood pressure and umbilical PI at baseline condition, at 10 and 120 min after reduction of fetal $\left[\mathrm{O}_{2}\right]$ a to below $2.0 \mathrm{mM}$ on the first day of experimentation (acute and subacute responses) and on the day prior to the diagnosis of fetal death or premature delivery (chronic responses). At $10 \mathrm{~min}$ after fetal $\left[\mathrm{O}_{2}\right]$ a had been reduced to below $2.0 \mathrm{mM}$ for the first time in all fetuses, mean fetal $\left[\mathrm{O}_{2}\right]$ a was $1.50 \mathrm{mM}$, and varied between 0.22 and 2.09 
$\mathrm{mM}$ (table 5.1). This reduction in fetal $\left[\mathrm{O}_{2}\right]$ a was accompanied by a decrease in $\mathrm{PaO}_{2}$ and base excess, whereas $\mathrm{pH}$ and $\mathrm{PaCO}_{2}$ had not significantly changed. In all fetuses, blood pressure increased, whereas heart rate showed the same wide variation as during baseline condition. On the basis of minor increases in four fetuses and minor decreases in the others, the mean umbillical PI remained unchanged. After $120 \mathrm{~min}$, the variable values had not changed, irrespective of base excess, which had further decreased in comparison with the value at 10 min, and of blood pressure, which no longer significantly differed in comparison with baseline condition. On the day prior to the diagnosis of fetal death or premature delivery, severe fetal hypoxemia was associated with moderate metabolic acidemia. Blood pressure had consistently increased in all fetuses, whereas heart rate and umbilical PI had remained unaffected, since the individual values did not show any consistent changes.

\section{DISCUSSION}

It is concluded from this study that a condition of prolonged hypoxemia and acidemia in fetal sheep, induced by embolization of the uterine microcirculation, is not associated with consistent changes in the umbilical PI. Clinical data on the relation between fetal oxygenation or acid-base balance and indices of umbilical artery blood flow velocity waveforms are scarce and limited to a select group of high-risk pregnancies (Ferrazzi et al., 1988). Since the origin of hypoxemia in normal as well as in growth-retarded human fetuses does not seem to be confined to only one mechanism, valid prediction of fetal oxygenation on the basis of indices of umbilical artery blood flow velocity waveforms merely depends on the strength of their correlation (Colton, 1974).

The purpose of this study was to evaluate the response of the umbilical PI to a condition of acute and prolonged hypoxemia and acidemia in fetal sheep. Embolizations of the uterine microcirculation were performed in order to reduce fetal oxygenation to predetermined levels. On the first day of experimentation, fetal $\left[\mathrm{O}_{2}\right]$ a was reduced to below $2.0 \mathrm{mM}$, since it was reported that this level of fetal hypoxia is associated with the emergence of metabolic acidosis (Peeters et al., 1979). In one fetus, [ $\left.\mathrm{O}_{2}\right]$ a acutely fell from 3.18 to $0.22 \mathrm{mM}$ in response to the first bolus injection with microspheres (table 5.1; figure 5.1, left upper panel). The gestational age of this fetus was 129 days, whereas the gestational ages at the time of experimentation in the other fetuses varied between 110 and 121 days. The sharp decline in fetal oxygenation in the older fetus may be due to a preexistent, limited placental reserve capacity. In addition, the values of umbilical PI in this fetus were substantially higher than those in 
the younger fetuses, whereas in the course of normal human and sheep pregnancy, waveform indices tend to decrease (van Vugt et al., 1987a; Newnham et $a l ., 1987)$. In the majority of fetuses, the first bolus injection did not result in an immediate decrease in fetal oxygenation. However, each second and next administration of microspheres reduced fetal arterial oxygen saturation by approximately $10 \%$.

The changes observed in the fetal acid-base status observed 10 minutes after fetal $\left[\mathrm{O}_{2}\right] \mathrm{a}$ had been reduced to below $2.0 \mathrm{mM}$ are compatible with those observed by others in experiments in which a comparable level of fetal hypoxemia was achieved by reduction of uterine blood flow (Skillman et al., 1985; Yaffe et al., 1987). It is also consistent with previous studies in our department and those of others, that acute fetal hypoxemia, when gradually induced, is associated with a wide variation in fetal heart rate, whereas abrupt and complete obstruction of uterine blood flow results in fetal bradycardia (Skillman $e t a l$, 1985; Yaffe et al., 1987; van Huisseling at al., 1989; Muijsers et al., 1990a). In response to acute hypoxemia, blood pressure increased in all fetuses, as a result of chemoreceptor activation (Hanson, 1988), while the umbilical PI remained unchanged. These findings are also consistent with the results of a previous study, in which fetal hypoxemia had been induced by progressive occlusion of the maternal common internal iliac artery (Muijsers et al., 1990a).

Evaluation of the variables 120 minutes after embolization showed a further increased metabolic acidemia. At the same time, an increase in fetal cardiac output is suggested by the mean (although statistically insignificant) changes in both heart rate and blood pressure. In spite of a possible increase in cardiac output and/or umbilical blood flow, there is no need for a change in fetal placental vascular resistance during moderate fetal hypoxemia (Parer, 1980; Yaffe et al., 1987; van Huisseling et al., 1990). These findings correspond with the absence of a change in the umbilical PI after two hours of moderate hypoxemia as observed in our experiments.

The prolonged hemodynamic response of the umbilical circulation in fetal lambs to embolization of the uterine circulation has been studied previously by Creasy et al. (1973) and by Clapp et al. (1980). Creasy et al. (1973) observed that umbilical blood flow in mildly hypoxemic, growth-retarded fetuses was lower than in normoxemic, normally grown controls. Clapp et al. (1980) correspondingly observed an increase in umbilical vascular resistance in mildly hypoxemic, growth-retarded fetuses versus a decrease in normal controls. Although the mechanisms of these observations remain unexplained, it would have been expected that the umbilical PI should correspondingly increase in response to uteroplacental embolization. However, the present study consider- 
ably differs from that of Creasy et al. (1973) and that of Clapp et al. (1980) concerning both the duration of the follow-up period and the severeness of hypoxemia.

Firstly, uteroplacental embolization over several weeks, without major changes in fetal oxygenation, as described by Creasy et al. (1973) and by Clapp et al. (1980), may have resulted in growth retardation on the basis of a deficit in nutritional placental transfer. Various reports suggest that uteroplacental embolization affects nutritional placental transfer to a more important amount than gaseous placental transfer (Harding et al., 1985; Charlton \& Johengen, 1987). The embolized condition may have accounted for fetal and placental growth retardation, associated with a decrease in placental vascular compliance and subsequently, an increase in impedance to umbilical blood flow.

Secondly, substantial hypoxemia, as present in our study, is generally accompanied by alpha- and/or beta-adrenergic stimulation (Jones et al., 1988). Bocking et al. (1988) investigated fetal blood flow distribution secondary to the prolonged restriction of uterine blood flow. They observed no changes in umbilical blood flow after a period of 48 hours of mild fetal hypoxemia. According to the authors, the maintenance of umbilical blood flow may be explained by prolonged beta-adrenergic stimulation, which appears to be of importance in maintaining umbilical blood flow during fetal hypoxemia (Parer, 1980; Parer, 1983). In addition, it has definitively been shown that umbilical vascular resistance remains unaffected secondary to beta-adrenergic stimulation (Adamson et al., 1989). Prolonged endocrine stimulation may have been present in our experiments too, since we observed a persistent increase in fetal arterial blood pressure (Jones et al., 1988). The hypothesis that mere hypoxemia results in increased impedance to umbilical blood flow is further undermined by the finding that chronic hypoxia in the guinea pig stimulates villous capillary branching, thus reducing impedance to umbilical blood flow (Scheffen et al., 1989).

In conclusion, this and other studies concerning the umbilical circulation support the point of view that the role of Doppler velocimetry in obstetrical management policy requires further examination (Neilson, 1987; Redman, 1989; Beattie \& Dornan, 1989). We suggest that knowledge on the mechanism of an increased umbilical PI will improve its clinical relevance. 


\section{CHAPTER 6}

\section{The effect of selective umbilical embolization on the common umbilical artery Pulsatility Index and umbilical vascular resistance in fetal sheep}

G.J.J.M. Muijsers, H. van Huisseling, T.H.M. Hasaart

Journal of Developmental Physiology, accepted for publication

\section{ABSTRACT}

In eight anesthesized fetal sheep (gestational age 112-127 days; term 147 days), embolization of the umbilical placental circulation was performed in order to evaluate the response of the umbilical artery Pulsatility Index to an exclusive increase in umbilical vascular resistance. Measurements were performed using a $20 \mathrm{MHz}$ pulsed Doppler transducer and an electromagnetic flow meter mounted on the common umbilical artery and catheters at the aortic trifurcation and in one of the umbilical veins. Umbilical vascular resistance was calculated according the Poiseuille equation as the ratio of aortic to umbilical venous pressure gradient and umbilical blood flow. Non-radioactive microspheres were administered at 15-minutes intervals through a catheter in one of the cotyledonary arteries, until fetal heart rate had decreased beneath 100 beats per minute or had become arrhythmic. The period of examination per fetus varied between 60 and 120 minutes, after which cardiac decompensation occurred. During this period, umbilical perfusion pressure (means in $\mathrm{mmHg} \pm \mathrm{SD}$ ) increased from $20.3 \pm 4.9$ to $28.1 \pm 4.7$ ( $<<0.01)$, umbilical blood flow ( $\mathrm{ml} / \mathrm{min})$ decreased from $342 \pm 127$ to $115 \pm 99$ ( $\mathrm{p}<0.01$ ), umbilical vascular resistance (mmHg.min/ml) increased from $0.065 \pm 0.022$ to $0.342 \pm 0.150(\mathrm{p}<0.01)$ and 
common umbilical artery Pulsatility Index increased from $0.97 \pm 0.23$ to $4.03 \pm$ $1.69(\mathrm{p}<0.01)$. Fetal heart rate did not change significantly $(168 \pm 33$ prior to cardiac decompensation versus $178 \pm 19$ at baseline condition). The linear correlation between common umbilical artery Pulsatility Index and umbilical vascular resistance varied between 0.83 and 0.99 and the average correlation was $0.93(\mathrm{p}<0.01)$. These data indicate that in human fetuses with normal heart rate, increased umbilical artery Pulsatility Index may result from increased umbilical vascular resistance.

\section{INTRODUCTION}

Doppler examination of the umbilical circulation has been subject to extensive study, and a variety of conclusions has been drawn. Most studies have evaluated the possible role of Doppler blood flow velocity waveform indices as a screening test or as a monitoring test for fetal growth and perinatal outcome (Reuwer et al., 1987; Trudinger et al., 1987a; van Vugt et al., 1988b; Beattie \& Dornan, 1989; Feinkind et al., 1989). Waveform indices, such as the Pulsatility Index (PI), A/B-ratio and Resistance Index (RI), are independent of the angle between the blood vessel of investigation and the emitted ultrasound. Furthermore, estimation of volume blood flow requires measurement of vessel diameter, which is not needed for calculation of waveform indices (Gill, 1985; Ruissen et al., 1987a).

It is assumed that indices of Doppler blood flow velocity waveforms reflect resistance to blood flow at the site of measurement, whilst the regulation of resistance is predominantly regulated downstream to this site. An increase in peripheral resistance would be associated with a relative decrease in diastolic blood flow velocity and thus, with an increase in waveform index. For waveform description, we use the PI according to Gosling, since this index contains the most specific waveform information comprised in one number (Gosling et al., 1971; Ruissen et al., 1987a).

On the basis of apparent biological importance and methodological simplicity, umbilical artery blood flow velocity waveform measurement is recommended as a tool for antenatal decision-making (Trudinger et al., 1987a; Rochelson et al, 1987a, 1987b), although others warn against too premature introduction of this technique into obstetric management (Neilson, 1987; Redman, 1989).

Various experimental studies have been performed to uncover the underlying mechanism of changes in fetal blood flow velocity waveforms, and to explore the relationship with vascular resistance. Noordam et al. (1987) found a significant correlation between lower aorta vascular resistance and PI in response to 
aortic embolization in 5 anesthesized fetal sheep. However, the possible effects of changes in fetal heart rate on both PI and calculated vascular resistance, were not discussed (Berman et al., 1978; Mulders et al., 1986; van Huisseling et al., 1989). The study of Trudinger et al. (1987b) in chronically instrumented fetal sheep also lacks numerical information on changes in fetal heart rate, while changes in the umbilical artery systolic to diastolic $(\mathrm{A} / \mathrm{B})$ ratio were small and did not consistently vary with changes in umbilical vascular resistance. Morrow et al. (1989) compared the diastolic to systolic (D/S) ratio with changes in umbilical vascular resistance, calculated from mean peak velocity, which was measured with the same Doppler transducer as used for D/S ratio determination. Their assumption that the common umbilical artery diameter remains constant following umbilical embolization is not valid, since embolization is accompanied with an increase in arterial blood pressure. The latter might result in an increase in vessel diameter, and thus, an underestimation of volumetric flow. In addition, the relationship between two correlating variables (PI and volume flow) can only be reliably determined using two, mutually independent, measuring instruments.

In all experimental studies afore-mentioned, umbilical vascular resistance was changed by administration of microspheres through a catheter in the lower fetal aorta, which was also used for measurement of umbilical inflow pressure. In the studies of Trudinger et al. (1987b) and Morrow et al. (1989) this may have resulted in an overestimation of umbilical perfusion pressure on the basis of an simultaneous increase in vascular resistance of the lower fetal body and thus, in an overestimation of umbilical vascular resistance.

The aim of this study was to examine the effect of selective umbilical placental embolization, via a catheter in a cotyledonary artery, on the common umbilical artery PI. We also examined the relationship between the common umbilical artery PI and calculated vascular resistance on the account that fetal heart rate remained within the normal range. A reduction in fetal heart rate is associated with both an increase in Doppler waveform indices on the basis of a pressure fall in the umbilical circulation and with a rise in umbilical-placental vascular resistance as calculated by the conventional formula assuming steady flow conditions (Berman et al., 1978). As a result, a correlation between the umbilical artery PI and umbilical vascular resistance during bradycardia does not include an virtual relationship between both variables, but merely a covariance (van Huisseling et al., 1989). 


\section{MATERIALS AND METHODS}

Surgery. Surgery was performed on eight pregnant sheep of the Dutch Texel breed between the 112th and 127 th day of gestation (term 147 days). Anesthesia was induced with pentobarbital infused intravenously and was maintained with $1 \%$ halothane in a $2: 1$ mixture of nitrous oxide and oxygen. After opening of the maternal abdomen through a paramedian incision, the uterus was incised over the fetal pelvis and the fetal lower body was exteriorized. A $20 \mathrm{MHz}$ pulsed Doppler transducer was mounted on the fetal aorta between the diaphragm and the origin of the coeliac trunk. Subsequently, an electromagnetic flow meter with an inner diameter of 4 or $5 \mathrm{~mm}$ (Skalar, Delft, The Netherlands) was applied around the common umbilical artery. Immediately next to the electromagnetic flow meter and on the common part of the umbilical artery as well, we mounted a $20 \mathrm{MHz}$ pulsed Doppler transducer. A polyvinyl catheter (outer diameter $1.6 \mathrm{~mm}$, inner diameter $0.8 \mathrm{~mm}$ ) was inserted into one of the femoral arteries and advanced precisely to the level of the aortic trifurcation. Another polyvinyl catheter was inserted via the femoral vein just into the inferior vena cava. After returning the fetus to the uterus, a cotyledonary artery and vein were cannulated and the catheters were advanced up to first order branches of the umbilical artery and vein, respectively. The uterus was closed in one layer, while the incision in the maternal abdominal wall, through which the catheters, Doppler and flow cables were exteriorized, was approximated using surgical clamps. The surgical and experimental procedures were approved by the local Animalls Medical Ethics Committee.

Experiments. The experiments started one hour after completion of the surgical procedure, while the ewe was in right lateral tilt position and general anesthesia was maintained. Placental embolization was preceded by administration of norepinephrine and angiotensin II during a 2 -hours experiment. The results of these studies are reported elsewhere. Placental embolization was started approximately 60 minutes after the final administration of norepinephrine or angiotensin II, when pressure, flow and Doppler measurements had returned to or come into line with baseline values. Measurements included fetal mean arterial pressure at the aortic trifurcation, umbilical venous pressure, fetal heart rate, on-line derived from the arterial pressure pulse using a tachometer, and umbilical blood flow. The zero reference values of the electromagnetic flowmeter were set prior to the experiment and afterwards. In 7 experiments, the difference between pre- and post-experimental reference values was less than $10 \mathrm{ml} / \mathrm{min}$ and therefore neglected. In one experiment the post-experimental reference value appeared to $60 \mathrm{ml} / \mathrm{min}$ higher than the pre-experimental one. The flow values measured in this experiment were therefore reduced by 60 
$\mathrm{ml} / \mathrm{min}$ before analysis. Pressure signals were determined with the zero point at the level of the ewe's abdomen. The pressure and flow signals were fed into amplifiers (Hewlett Packard 8800 Series, Hewlett Packard Company, Andover, Massachusetts, USA), displayed on monitor, recorded on eight-channel stripchart recorder and stored on magnetic tape. Mean values of fetal heart rate, fetal mean arterial pressure, umbilical venous pressure and umbilical blood flow were calculated by computer over 10-seconds periods. Blood flow velocity waveforms in the common umbilical artery were obtained by means of the implanted $20 \mathrm{MHz}$ pulsed Doppler transducer (545C-4 Directional Pulsed Doppler Flowmeter, University of Iowa, Iowa City, USA). The frequency shifted signals were stored in quadrature on audiotape and processed by a spectrum analyser (Doptek spectrum analyser, Doptek Ltd., Chicester, England). The PI, as used in our experiments for waveform description, is defined as the difference between the maximum and minimum frequency shift divided by the mean frequency shift of the maximum envelope curve throughout the cardiac cycle. The parameter calculations were performed off-line by drawing the maximum envelope curves of the frequency-time spectra with a handguided light-pen (Ruissen et al., 1987a).

After measurement of baseline values, $1.5 \times 10^{6}$ non-radioactive mucopolysaccharide microspheres of approximately 20 micron (Sephadex, Uppsala, Sweden) were introduced into the umbilical circulation via the catheter in the cotyledonary artery every 15 minutes until fetal death, extreme bradycardia (fetal heart rate beneath 100 beats per minute) or arrhythmia occurred. All measurements, including fetal heart rate, fetal mean arterial pressure, umbilical venous pressure, umbilical blood flow and umbilical artery PI were taken during stable readings just before a next administration of microspheres.

Calculations and data analysis. Fetal heart rate, fetal mean arterial pressure, umbilical venous pressure and umbilical blood flow were averaged over 10 -seconds periods using a personal computer program. The common umbilical artery PI was calculated as a mean value, averaged over five consecutive cardiac cycles within the same 10-seconds period "Umbilical perfusion pressure was defined as the numerical difference between fetal mean arterial pressure and umbilical venous pressure. Umbilical vascular resistance was calculated according the Poiseuille equation as umbilical perfusion pressure divided by umbilical blood flow.

The average linear trend of the variables per hour was determined by averaging the individual linear regression slopes over all fetuses. The trend of each variable was statistically evaluated by Wilcoxon's signed rank test. The mean change in the variable values from baseline condition to the condition prior to fetal bradycardia or arrythmia was calculated and tested using a Wilcoxon 
matched pairs signed rank test. The average correlation between umbilical artery PI and umbilical vascular resistance was calculated by averaging the correlation coefficients from the eight individual fetal sheep, and was tested using Wilcoxon"s signed rank test.

\section{RESULTS}

The period of examination per fetus varied between 60 and 120 minutes. Fifteen minutes after this period, fetal heart rate was beneath 100 beats per minute or arrhythmic in each of the eight animals under investigation. This
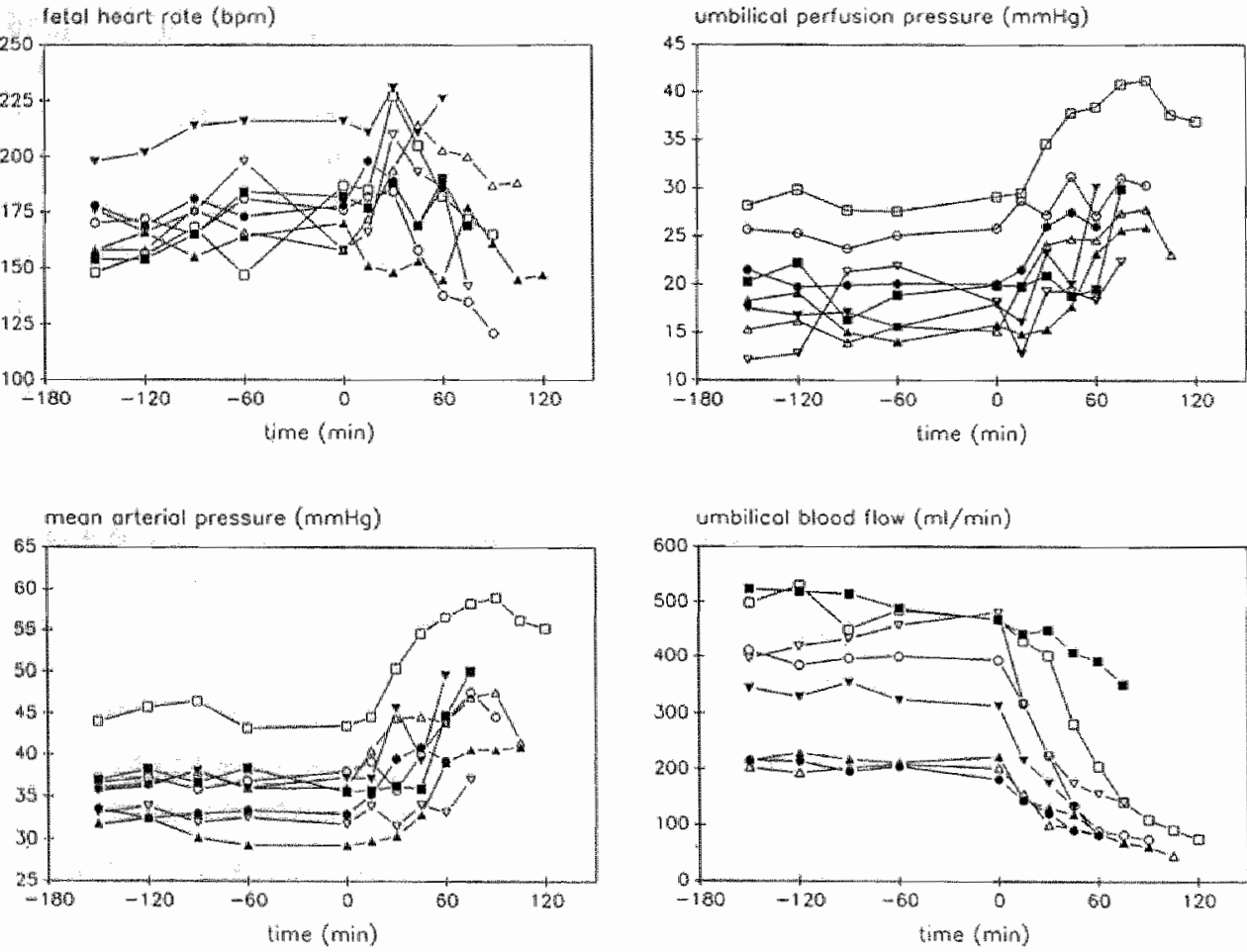

Figure 6 1. The course of fetal heart rate (upper panel) and mean arterial pressure (lower panel) measured at baseline conditions during a preceding experiment and immediately prior to repetitive cotyledonary embolizations at 15-minutes intervals.

Figure 6-2. The course of umbilical perfusion pressure (upper panel) and umbilical blood flow (lower panel) measured at baseline conditions during a preceding experiment and immediately prior to repetitive cotyledonary embolizations at 15-minutes intervals. 
bradycardia and arrhythmia were regarded as features of cardiovascular breakdown and accompanied by a rapid decrease in mean arterial pressure in all fetuses, and eventually, by fetal death. After emergence of the individual cardiovascular break-point, no further measurements were performed.

Figures 6.1 to 6.3 show the course of the variables measured or calculated as a function of time in each of the 8 fetuses. The baseline values from the preceding experiment are depicted on the time scale at $-180,-120,-90$ and -60 minutes. The baseline value of the embolization experiment is depicted on the time scale at 0 minutes.

Since the duration of the experiment varied per fetus, longitudinal analysis was performed by calculating linear regression lines between all variables and time for each fetus. The average linear trends of the variables per hour

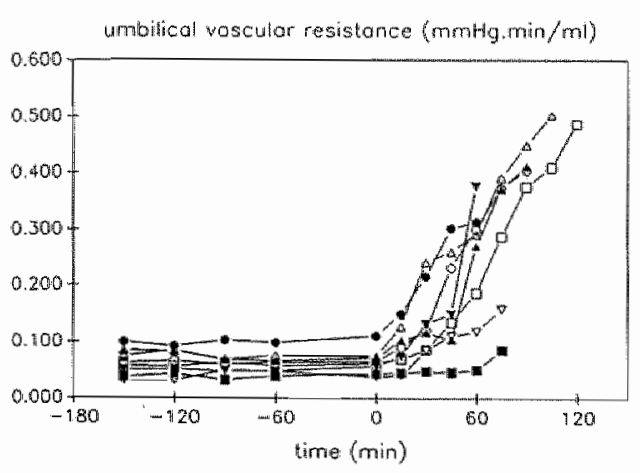

umbülical ortery pulsotility index

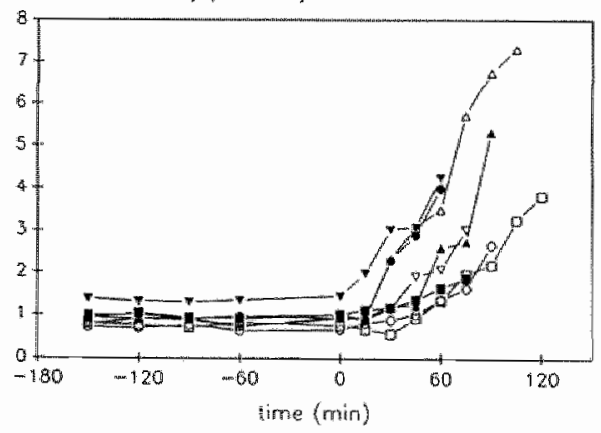

Figure 6-3. The course of umbilical wascular resistance (upper panel) and umbilical artery PI (lower panel) measured at baseline conditions during a preceding experiment and immediately prior to repetitive cotyledonary embolizations at 15 -minutes intervals. were determined by averaging the linear regression slopes over all fetuses (table 6.1).

Table 6.2 lists the mean variable values at baseline condition and prior to termination of the experiment. It is shown that fetal heart rate did not significantly change during the period of examination. Umbilical perfusion pressure increased, due to an increase in mean arterial pressure and no change in umbilical venous pressure. Umbilical blood flow almost consistently decreased with each additional bolus of microspheres. In the same way, umbilical vascular resistance increased during embolization. The common umbilical artery PI did not consistently increase until the third bolus of microspheres was introduced into the umbilical circulation. Thereafter, each bolus consistently resulted in an increase in PI.

Figure 6.4 shows some of the changes observed in one of the fetuses. This fetus is in the other 
Table 6.1. Average linear trend of the variables per hour, determined by averaging the individual linear regression slopes. Statistical analysis by Wilcoxon signed rank test.

variable

fetal hesrt rate

fetal mean arterial pressure

umbilical venous pressure

umbilical perfusion pressure

umbilical blood flow

umbilical wascular resistance

umbilical antery PI average trend/hour

$\begin{array}{ll}-7.5 \mathrm{bpni} & \mathrm{ns} \\ +7.2 \mathrm{mmHg} & <0.01 \\ +1.2 \mathrm{mmHg} & \mathrm{ns} \\ +6.1 \mathrm{mmHg} & <0.01 \\ -161 \mathrm{~mL} / \mathrm{min} & <0.01 \\ +0.20 \mathrm{mmHg} . \mathrm{min} / \mathrm{ml} & <0.01 \\ +2.20 & <0.01\end{array}$

Table 6.2. Comparison of the mean variable values $( \pm S D)$ at the end of the experiment with those at baseline condition by Wilcoxon's matched pairs signed rank test.

\begin{tabular}{lccc}
\hline variable & $\begin{array}{l}\text { baseline } \\
\text { condition }\end{array}$ & $\begin{array}{l}\text { end of the } \\
\text { experiment }\end{array}$ & p-value \\
\hline fetal heart rate (bpm) & $178 \pm 19$ & $168 \pm 33$ & $\mathrm{~ns}$ \\
fetal mean arterial pressure (mmHg) & $35.6 \pm 4.3$ & $44.8 \pm 6.3$ & $<0.01$ \\
umbilical venous pressure (mmHg) & $15.3 \pm 3.2$ & $16.8 \pm 2.6$ & $\mathrm{~ns}$ \\
umbilical perfusion pressure (mmHg) & $20.3 \pm 4.9$ & $28.1 \pm 4.7$ & $<0.01$ \\
umbilical blood flow (ml/min) & $342 \pm 127$ & $115 \pm 99$ & $<0.01$ \\
umbilical vascular resistance (mmHg.min/ml) & $0.065 \pm 0.022$ & $0.342 \pm 0.150$ & $<0.01$ \\
umbilical artery PI & $0.97 \pm 0.23$ & $4.03 \pm 1.69$ & $<0.01$ \\
\hline
\end{tabular}

figures represented by upward pointing open triangles. The first panel (from left to right) depicts the waveform and the parameter values at baseline condition. The second panel represents the waveform and the parameter values 15 minutes later, following the first embolization. A small decrease in PI was observed, although vascular resistance had increased and blood flow had decreased. This decrease in PI is probably due to an increase in fetal heart rate. The third panel shows the waveform after two additional embolizations at 45 minutes. In spite 
common umbilical artery blood flow velocity waveforms
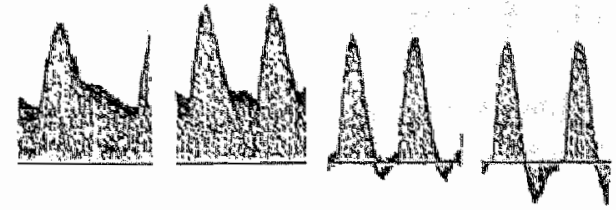

$\begin{array}{lllll}\text { common umbilical PI } & 0.95 & 0.92 & 3.09 & 6.74 \\ \text { resistance (mmHg.min/m) } & 0.074 & 0.127 & 0.260 & 0.448 \\ \text { blood flow (m//min) } & 203 & 157 & 95 & 62 \\ \text { perfusion pressure (mmHg) } & 15.1 & 19.9 & 24.7 & 27.8 \\ \text { fetal heart rate (bpm) } & 158 & 172 & 214 & 187\end{array}$

Figure 6.4. Common umbilical artery blood flow velocity waveforms and concurrent values of the hemodynamic variables at various conditions relative to umbilical embolization.

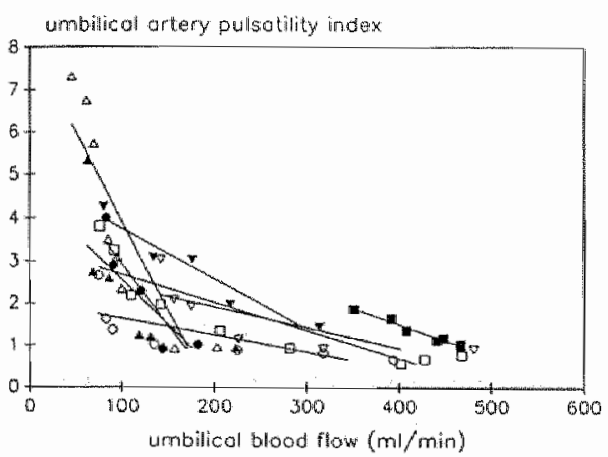

urmbilical ortery pulsotiliby index

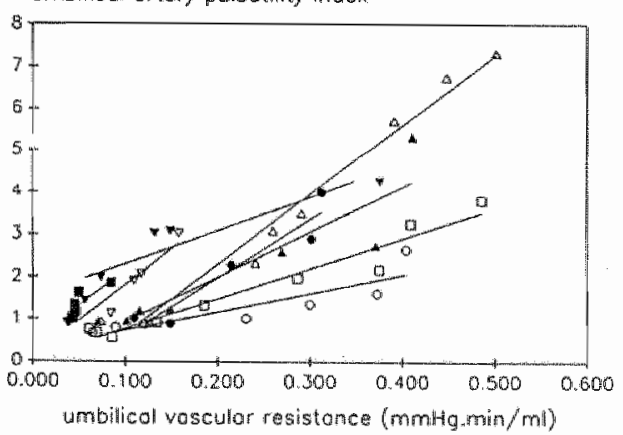

Figure 6.5. Upper panel: linear regression lines $(n=8)$ expressing the relationship between common umbilical artery PI and umbilical blood flow, altered by umbilical embolization. Lower panel: linear regression lines $(n=8)$ expressing the relationship between common umbilical artery PI and umbilical vascular resistance, altered by umbilical embolization. of a further increase in fetal heart rate, there was an increase in PI, in association with an increase in vascullar resistance and a decrease in blood flow. The fourth panel shows the condition prior to near cardiac failure and death.

The relationship between common umbilical artery PI and umbilical blood flow in this umbilical embolization model is depicted in figure 6.5 upper panel. Figure 6.5 lower panel, depicts the relationship between common umbilical artery $P I$ and umbilical vascular resistance. In all fetuses, there was a significant correlation, with correlation coefficients between 0.83 and 0.99 . The average correlation was 0.93 ( $p<0.01)$, suggesting that umbilical vascular resistance is an important determinator of the umbilical artery blood flow velocity waveform. 


\section{DISCUSSION}

It is concluded from this study that embolization of the umbilical circulation can result in a sharp rise of the common umbilical artery PI, which is strongly associated with umbilical vascular resistance. Since changes in fetal heart rate were small and insignificant, this parameter cannot have accounted for the more than 4-fold mean increase observed in common umbilical artery PI. This study was undertaken in an anesthesized sheep model since it had appeared in pilot experiments that umbilical embolization in chronically instrumented fetal sheep invariably resulted in a severe decrease in fetal heart rate, one of the determinators of umbilical artery blood flow velocity waveform indices in both human and sheep fetuses (Mulders et al., 1986, van Huisseling et al., 1989). This embolization-induced bradycardia appeared to be irreversible and eventually, resulted in fetal death. The same findings were reported by Morrow et al. (1989), whereas waveform index changes were relatively small when fetal heart rate was reported to be unchanged (Trudinger et al., 1987b). Berman et al. (1978) already drew attention to the subject of calculating vascular resistance during bradycardia. We agree with their statement that a decrease in umbilical blood flow associated with a decrease in heart rate cannot be interpreted as an active increase in umbilical-placental vascular resistance. An increase in vascular resistance does not identify the nature, cause or exact site of increased dissipation of the energy of blood flow within the vascular bed (Milnor, 1972).

In this study, in which the cotyledonary circulation was selectively embolized using 20 micron microspheres, it is suggested that a dose-related part of the fetal placental arterioles has been completely obstructed, since the diameters of the fetal villous capillaries in sheep from 100 days" gestation onwards, range between 2 and 8 micron (Barcroft \& Barron, 1946).

A strong correlation was found between the umbilical artery PI, calculated on the basis of pulsatile changes in blood flow velocity, and umbilical vascular resistance, calculated on the basis of mean arterial-venous pressure gradients and mean flows. This finding however, does not imply that changes in Doppler blood flow velocity waveforms only depend on changes in vascular resistance, which refers to changes in vessel caliber. Blood flow velocity waveforms may, among others, also be related to other hemodynamic characteristics, such as vascular compliance, which refers to vascular distensibility; viscosity and density, which refer to the physical properties of blood; and wave reflections, which refer to the discontinuity of the lumen area and the thickness and viscoelasticity of the vessel walls. 
The shortcomings of vascular resistance for description of pressure-flow relationships may be overcome by computation of vascular impedance, defined as the ratio of pulsatile pressure to pulsatile flow. Before impedance can be calculated from the pressure and flow pulsations, the waveforms must be resolved into their sinusoidal components by Fourier analysis (Milnor, 1972; O'Rourke, 1982).

In human studies it is reported that blood flow velocity waveform changes can precede changes in the fetal heart rate pattern for several weeks (Reuwer $e t$ al., 1984b; Trudinger et al., 1985; Erskine \& Ritchie, 1985; Cameron et al., 1988). This implies that waveform changes in the human fetus probably develop very gradually, so that the fetus has time to adjust circulation and metabolism to a state of relative high impedance to umbilical blood flow.

Various studies were performed to examine the relationship between umbilical artery blood flow velocity waveform indices and placental pathology. It is suggested that an increase in umbilical artery blood flow velocity waveform indices is associated with a decreased number of small muscular arteries in the tertiary stem villi (Giles et al., 1985; McCowan et al., 1987; Bracero et al., 1989). The pathogenesis of these findings, which have not been reported earlier (Fox, 1986) in relation to fetal growth retardation, distress or death, is still unknown. Future studies on placental pathology should therefore preferably focus on the relation between abnormal fetal villous vascularization and abnormal transformation of maternal spiral arteries into uteroplacental vessels, observed in pregnancies complicated by pre-eclampsia and small-for-gestationalage infants (Khong et al., 1986). 


\title{
CHAPTER 7
}

\section{Regional umbilical embolization in sheep results in discordant blood flow velocity waveforms at the proximal and distal segments of the fetoplacental circulation}

\author{
G.J.J.M. Muijsers, H. van Huisseling, T.H.M. Hasaart
}

Submitted for publication

\section{ABSTRACT}

Regional embolization of the placental circulation was performed in three anesthesized fetal sheep by retrograde administration of microspheres in a cotyledonary branch of one of the umbilical arteries. Blood flow velocity waveforms were registered from the abdominal aorta in 1 fetus, from the common umbilical artery in 2 fetuses (proximal waveforms), and from a major cotyledonary artery supplying the embolized placental region in each of the 3 fetuses (distal waveforms). Among the individual fetuses, a corresponding degree of regression was found between the decrease in umbilical blood flow and increase in the PI of the proximal waveforms (coefficient of variation: 0.19). In contrast, the degree of regression between the decrease in umbilical blood flow and increase in the PI of the distal waveforms, varied per fetus (coefficient of variation: 0.79). It is suggested that in human fetuses, in the case of discordant umbilical artery blood flow velocity waveforms, the best information on fetoplacental circulatory competence, is provided by the aorta blood flow velocity waveform. 


\section{INTRODUCTION}

Various studies have been performed to examine the relationship between umbilical artery blood flow velocity waveform indices and placental pathology. It is suggested that an increase in umbilical artery blood flow velocity waveform indices is associated with a decreased number of small muscular arteries in the tertiary stem villi (Giles et al., 1985; McCowan et al., 1987; Bracero et al., 1989). In a case report by Trudinger and Cook (1988), a fetus is described in which serial umbilical artery blood flow velocity waveform examinations consistently revealed a normal pattern in the one, and an abnormal pattern in the other umbilical artery. Placental examination after elective delivery at 33 weeks showed that one of the umbilical arteries coursed across the surface of the placenta to an infarcted lobe, whereas the other umbilical artery supplied an unaffected part of the placenta. Microscopic examination showed extensive perivillous fibrin deposition and villous necrosis close to the uterine surface. Considerable syncytial knotting and sclerosis of the small arteries in the tertiairy villi were observed in the subchorionic region of the infarcted lobe, while the umbilicall vessells on the chorionic plate were normal.

Recently, various studies have been performed to examine the effect of fetal placental embolization on umbilical artery blood flow velocity waveforms in sheep (Noordam et al., 1987; Trudinger et al., 1987b; Morrow et al., 1989). However, in all studies, embolization was performed by introduction of microspheres from the fetal aorta, probably resulting in diffuse obstruction of the placental microcirculation as well as that of the lower fetal body.

The aim of this study was to examine the effect of massive regional cotyledonary embolization on the flow velocity waveforms of a major cotyledonary artery supplying an embolized region as opposed to the blood flow velocity waveforms in arteries supplying the whole placenta, such as the aorta and, in sheep, the common umbilical artery. Furthermore, we examined the relationship between the Pulsatility Index (PI) of these waveforms and umbilical blood flow and umbilical vascular resistance.

\section{MATERIALS AND METHODS}

Surgery. Surgery was performed on three pregnant sheep of the Dutch Texel breed with gestational ages of 113,112 and 117 days, respectively (term 147 days). Anesthesia was induced with pentobarbital infused intravenously, and was maintained with $1 \%$ halothane in a $2: 1$ mixture of nitrous oxide and oxygen. After opening of the maternal abdomen through a paramedian incision, 
the uterus was incised over the fetal pelvis and the fetal lower body was exteriorized. The fetal aorta was prepared via a retroperitoneal aprroach and a $20 \mathrm{MHz}$ pulsed Doppler transducer was mounted at the level between the diaphragm and the origin of the coeliac trunk. Subsequently, an electromagnetic flow meter with an inner diameter of 4 or $5 \mathrm{~mm}$ (Skalar; Delft, The Netherlands) was applied around the common umbilical artery. A $20 \mathrm{MHz}$ pulsed Doppler transducer was mounted immediately next to the electromagnetic flow meter and on the common part of the umbilical artery as well. A polyvinyl catheter (outer diameter $1.6 \mathrm{~mm}$, inner diameter $0.8 \mathrm{~mm}$ ) was inserted into one of the femoral arteries and advanced precisely to the level above the aortic trifurcation. Another polyvinyl catheter was inserted via the femoral vein just into the inferior vena cava. After returning the fetus to the uterus, a cotyledonary artery and vein were cannulated and the catheters were advanced up to major coty ledonary vessels. Approximately 2-3 centimeters away from the catheter tip in fetal direction, a pulsed Doppler transducer was mounted on a primary branch of one of the umbilical arteries. The uterus was closed in one layer, while the incision in the maternal abdominal wall, through which the catheters, Doppler and flow leads were exteriorized, was approximated using surgical clamps. The surgical and experimental procedures were approved by the local Animals Medical Ethics Committee.

Experiments. The experiments started one hour after completion of the surgical procedure, while the ewe was in right lateral tilt position and general anesthesia was maintained. Placental embolization was preceded by administration of norepinephrine and angiotensin II during a two-hour experiment. The results of these studies will be reported elsewhere. Placental embolization was started approximately one hour after the last administration of the two mentioned drugs, when pressure, flow and Doppler measurements had returned to or had come into line with baseline values. Measurements included fetal mean arterial pressure at the aortic trifurcation, cotyledonary venous pressure, fetal heart rate, on-line derived from the arterial pressure pulse using a tachometer, and umbilical blood flow. Pressure signals were determined with the zero point at the level of the ewe's abdomen. The pressure and flow signals were fed into amplifiers (Hewlett Packard 8800 Series, Hewlett Packard Company, Andover, Massachusetts, USA), displayed on monitor, recorded on strip-chart recorder and stored on magnetic tape. Mean values of heart rate, mean arterial pressure, cotyledonary venous pressure and umbilical blood flow were calculated by computer over 10 -seconds periods. Blood flow velocity waveforms were abtained from a major cotyledonary artery and, dependent of the quality of the Doppler signal, from either the abdominal aorta (in 1 fetus) or the common umbilical artery (in 2 fetuses), using the implanted $20 \mathrm{MHz}$ pulsed Doppler 
transducers (545C-4 Directional Pulsed Doppler Flowmeter, University of lowa, lowa City, USA). The frequency shifted signals were stored in quadrature on magnetic tape and processed by a spectrum analyser (Doptek Ltd., Chicester, England). The PI, as used in our experiments for waveform description, is defined as the difference between the maximum and minimum frequency shift divided by the mean frequency shift of the maximum envelope curve throughout the cardiac cycle. The PI calculations were performed off-line by drawing the maximum envelope curves of the frequency-time spectra with a handguided light-pen (Ruissen et al., 1987a).

After measurement of baseline values, $1.5 \times 10^{6}$ non-radioactive mucopolysaccharide microspheres of approximately 20 micron (Sephadex, Uppsala, Sweden) were introduced into the umbilical circulation via the catheter in the cotyledonary artery every 15 minutes until fetal death, extreme bradycardia (fetal heart rate beneath 100 beats per minute) or arrhythmia occurred. All fetal measurements, including heart rate, mean arterial pressure, cotyledonary venous pressure, umbilical blood flow, and blood flow velocity waveforms were taken during stable readings prior to a next administration of microspheres.

Calculations and data analysis. Heart rate, mean arterial pressure, umbilical venous pressure and umbillical blood flow were averaged over 10 -seconds periods using a personal computer program. The PI values were calculated as a mean value, averaged over five consecutive cardiac cycles within the same 10 -seconds period. Umbilical perfusion pressure was defined as the numerical difference between fetal mean arterial pressure and venous cotyledonary pressure. Umbilical vascular resistance was calculated according the Poiseuille equation as umbilical perfusion pressure divided by umbilical blood flow.

The small number of fetuses involved in the study and the wide interfetal variation hindered application of parametric as well as nonparametric statistical procedures. Therefore, average linear trends were calculated of the regressions between:

- the PI in the proximal waveforms and umbilical blood flow

- the PI in the distal waveforms and umbilical blood flow

- the PI in the proximal waveforms and umbilical vascular resistance

- the PI in the distal waveforms and umbilical vascular resistance

To get insight in the degree of consistency among the individual fetuses, we calculated the coefficient of variation of the average linear trends. A low coefficient of variation implies a high consistency in the regression between two variables and vice versa. 


\section{RESULTS}

The period of examination per fetus varied between 60 and 90 minutes. Fifteen minutes after this period, fetal heart rate was beneath 100 beats per minute or arrhythmic in each of the three animals under investigation. This bradycardia or arrhythmia was accompanied by a rapid decrease in mean arterial pressure in all fetuses and eventually, by fetal death.

Table 7.1 lists the mean variable values at baseline condition and prior to termination of the experiment. It is suggested that regional umbilical embolization results in a decrease in umbilical blood flow and increases in the PI's of the distal and proximal fetoplacental waveforms, umbilical perfusion pressure and umbilical vascular resistance.

Table 7.1. Mean values and ranges of some variables before embolization and at the end of the experiment.

\begin{tabular}{|c|c|c|c|c|}
\hline \multirow[t]{2}{*}{ variable } & \multicolumn{2}{|c|}{ pre-embolization } & \multicolumn{2}{|c|}{ post-embolization } \\
\hline & mean & range & mean & range \\
\hline PI in abdominal aorta or umbilical artery & 1.26 & $0.94-1.82$ & 4.00 & $2.67-5.32$ \\
\hline PI in cotyledonary artery & 1.00 & $0.83-1.27$ & 52.37 & $5.08-112.41$ \\
\hline fetal heart rate (bpm) & 177 & $170-182$ & 172 & $161-186$ \\
\hline umbilical perfusion pressure $(\mathrm{mmHg})$ & 18.6 & $15.8-20.1$ & 27.3 & $25.9-29.9$ \\
\hline umbilical blood flow (ml/min) & 291 & $182-467$ & 166 & $83-351$ \\
\hline umbilical vascular resistance (mmHg.min/ml) & 0.075 & $0.043-0.110$ & 0.270 & $0.085=0.411$ \\
\hline
\end{tabular}

Figure 7.1 shows the regression lines between:

- the PI in the abdominal aorta or common umbilical artery and the percentage of umbilical blood flow with respect to the baseline value (upper panel left)

- the PI in a major cotyledonary artery supplying an embolized placental region and the percentage of umbilical blood flow (upper panel right)

- the PI in the abdominal aorta or common umbilical artery and the percentage of umbilical vascular resistance (lower panel left)

- the PI in a major cotyledonary artery supplying an embolized placental region and the percentage of umbilical vascular resistance percentage (lower panel right). 


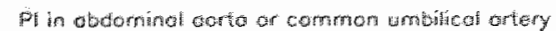

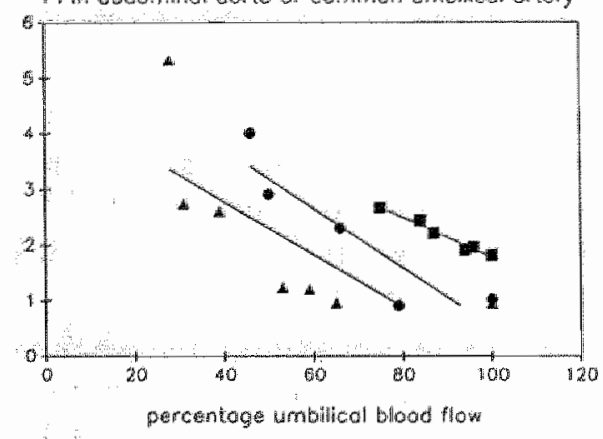

Pil ablominal oorto ar carmon umbificall ortery

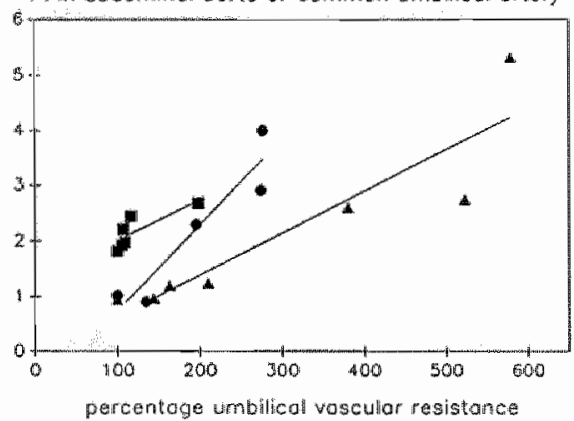

Pin cotyledonoy ortery

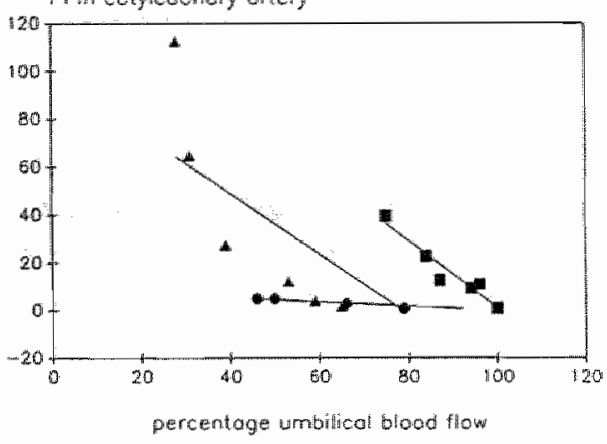

$\mathrm{Pl}$ in colyledonory artery

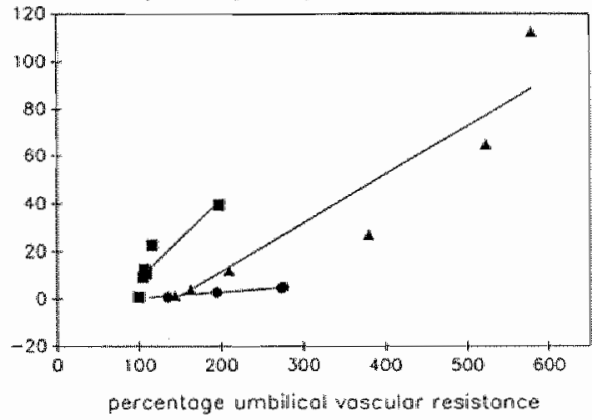

Figure 7.1. Linear regression lines expressing the relationship between PI measurements at distinct sites of the fetoplacental circulation and percentage umbilical blood flow (upper panels) as well as the percentage umbilical vascular resistance (lower panels). In the left panels, the circles and triangles refer to PI measurements in the common umbilical artery, while the squares refer to PI measurements in the abdominal aorta.

Table 7.2. Average linear trends of proximal and distal waveform PI"s as opposed to the percentage of total umbinical blood flow and umbilical vascular resistance at baseline condition. The coefficient of variation (COV) provides an indication the consistency of this trend among the fetuses.

PI in aorta or umbilical artery vs. \% umbilical blood flow

$\begin{array}{lll}-0.0453 & 0.0086 & 0.19\end{array}$

PI in cotyledonary artery PI vs. \% umbilical blood flow

$-0.918$

0.724

0.79

PI in aorta or umbilical artery vs. \% umbilical vascular resistance

0.0099

$0.0044 \quad 0.44$

PI in cotyledonary artery vs. \% umbilical vascular resistance

0.187

0.154

0.82 
Note that placental embolization can result in extreme PI values in the cotyledonary arteries. In contrast, the relationship between the umbilical blood flow percentage and the PI in the abdominal aorta or common umbilical artery, suggests a more consistent trend among the fetuses, while the PI values measured resemble to those observed in human pregnancy.

Table 7.2 lists the average linear trends (mean coefficient of the regression lines as depicted in figure 7.1), standard deviations and coefficients of variation for the regressions studied. It is clearly shown that the coefficient of variation of the mean coefficient describing the relationship between the proximal waveform PI and umbilical blood flow is lower than that describing the relationship between distal waveform and umbilical blood flow.

Figure 7.2 (from left to right downwards) shows some of the changes observed in the cotyledonary artery (lower waveforms) as opposed to the changes observed in the common umbilical artery (upper waveforms) in one of the fetal lambs (in figure 7.1 represented by triangles). The simultaneous presence of two velocity-time spectra in one display are the result of simultaneous transformation of the common umbilical and cotyledonary artery Doppler signals. Assuming that no time differences occurred in the electronic pathways during registration or transformation, the delay between both pulse waves was 20-30 $\mathrm{ms}$. The distance between the common umbilical and the cotyledonary artery varied between 30 and $40 \mathrm{~cm}$. As a result, the estimated velocity of propagation of the pressure pulse through the umbilical artery varied between 10 and $20 \mathrm{~m} / \mathrm{s}$, which corresponds with adult values for pressure pulse transmission in large arterial branches from the aorta (McDonald, 1974). The upper panel left depicts waveforms prior to embolization. The upper panel right and middle panel left show the waveforms after 2 and 3 embolizations. It is shown that marked changes in the artery nearby the embolized region is associated with absence of changes in the artery supplying the whole placenta. After 4 embolizations (middle panel right), a dicrotic notch occurred in the common umbilical artery blood flow velocity waveform, indicating increased impedance (Burns, 1988a). This condition was associated with a doubling of umbilical vascular resistance when compared with baseline condition, but not yet a relevant change in the common umbilical artery PI. After 5 embolizations, a nearly four-fold increase in resistance measured in the common umbilical artery was associated with a marked reduction in the diastolic blood flow velocity (lower panel left). Eventually, after 6 embolizations, reverse blood flow velocity also occurred in the common umbilical artery (power panel right). By that time, total umbilical blood flow had decreased by more than 70 percent. 

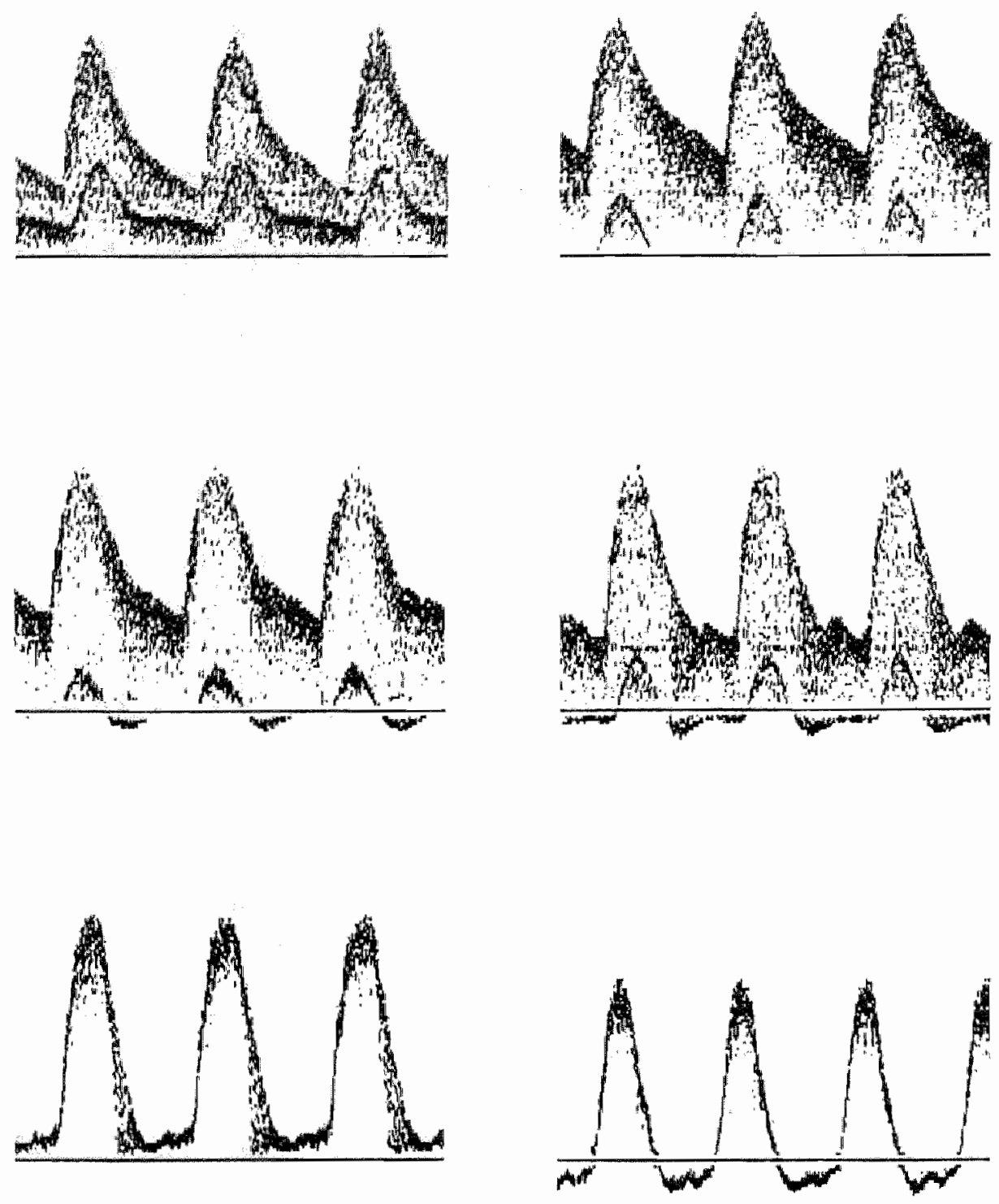

Figure 7.2. Consecutive changes observed in the blood velocity waveforms of the common umbilical artery (upper waveforms) and cotyledonary artery (lower waveforms) following regional umbilical embolization in one of the fetal lambs. 


\section{DISCUSSION}

Discordant umbilical artery blood flow velocity waveforms are observed more frequently as might be excepted on the basis of a single case report (Trudinger \& Cook, 1988). Harper \& Murnaghan (1990) described a series of 7 fetuses with discordant umbilical artery blood flow velocity waveforms. Extensive infarcts were found in the placentas of 6 fetuses, while an old retroplacental clot was found behind the placenta of the 7 th fetus.

The results of our experimental findings indicate that regional umbilical embolization in sheep is associated with abnormal velocity waveforms in supplying arteries. The amount of abnormality appears to be related to the relative change in impedance to blood flow at the site of measurement. In arteries nearby a region with increased impedance to blood flow, these changes may exceed and preceed the changes observed in arteries, such as the fetal aorta, which also supply other (placental) regions, in which impedance to blood flow has not changed. Moreover, it is suggested that local placental hypoperfusion can be compensated by preferential hyperperfusion of other placental regions (Bissonnette \& Farrell, 1973). The mechanisms by which such a compensatory hyperperfusion may occur are an increase in total placental perfusion pressure or a decrease in local placental vascular resistance. The regulation of such mechanisms however, is unknown.

In a series of eight fetal sheep undergoing selective umbilical embolization, it was observed that an increase in common umbilical artery PI was preceded by a consistent decrease in total umbilical blood flow and increase in umbilical vascular resistance (Muijsers et al., 1990b). This implies that the PI might be not so sensitive to small changes in blood flow or blood flow impedance.

Despite the potential insensitivity of blood flow velocity waveform indices in the assessment of small or moderate changes in blood flow, it appears that severe decrements can be identified more easilly. However, if abnormal waveforms are observed in only one of both umbilical arteries, this finding appears to provide little information on total fetoplacental blood flow impedance (Trudinger \& Cook, 1990). In such cases, information on impedance to umbilical blood flow might be derived from waveform characteristics in the fetal aorta, since approximately two third of the blood flow through the fetal thoracic aorta is diverted to the umbilical circulation (Rudolph \& Heymann, 1970; Anderson et al., 1981).

In conclusion, the results of this experimental study in fetal sheep suggests, that the significance of abnormal waveforms originating from only one of both umbilical arteries in human fetuses, can be evaluated by comparison with the waveform characteristics in the fetal aorta. 


\section{CHAPTER 8}

\section{The effects of hypertensive drugs on}

parameters derived from blood flow velocity waveforms in the systemic circulation of fetal sheep

G.J.J.M. Muijsers, C.J. Ruissen, H. van Huisseling, T.H.M. Hasaart

Submitted for publication

\section{ABSTRACT}

In eight fetal sheep under anesthesia, administration of norepinephrine and angiotensin II was performed to examine the relationship between fetal mean arterial pressure and the Pulsatility Index of the fetal aorta blood flow velocity waveform above the level of the visceral organs (aorta PI). It was hypothesized that the aorta PI would correlate with fetal mean arterial pressure. Measurements were performed using a $20 \mathrm{MHz}$ Doppler transducer on the subdiaphragmatical aorta and a catheter inserted via the femoral artery into the abdominal aorta. Further instrumentation included a Doppler transducer and an electromagnetic flowmeter on the common umbilical artery, a catheter in the inferior vena cava, and catheters in a cotyledonary artery and vein. Fetal hypertension was induced by bolus administration of norepinephrine and angiotensin II or by norepinephrine infusion. Adjustment of the depth resolution of the Doppler transducer on the common umbilical artery made it possible to study external iliac artery blood flow velocity waveforms in four fetal lambs, whereas aorta blood flow velocity waveforms were recorded in seven fetal lambs. The measurements of external iliac artery PI and mean arterial pressure were characterized by a linear regression with correlation coefficient of 0.72 , 
whereas the measurements of aorta PI and mean arterial blood pressure were characterized by a linear regression with a correlation coefficient of 0.40 . However, notable changes were observed in the aorta blood flow velocity waveform, which were not expressed by a substantial increase in the aorta PI. These changes can be described as a narrowing of the systolic peak and an earlier occurrence of the first dicrotic notch. The correlation coefficient of the linear regression line between the cardiac cycle time until occurrence of the first dicrotic notch in the aorta blood flow velocity waveform and mean arterial blood pressure was -0.77 . It is concluded that parameters derived from the aorta blood flow velocity waveform correlate with fetal mean arterial pressure in response to hypertensive drugs in fetal sheep.

\section{INTRODUCTION}

In the course of fetal life, blood pressure increases and placental vascular resistance decreases (Dawes, 1962). The increase in blood pressure can be explained by a concurrent increase in systemic vascular resistance during fetal development. The increase in systemic vascular resistance is parallelled by an increase in thoracic aorta PI, as measured with Doppler ultrasound (van Vugt et al., 1987b), whereas the decrease in placental vascular resistance is parallelled by a decrease in umbilical artery PI (Reuwer et al., 1984; Erskine \& Ritchie, 1985; van Vugt et al., 1987a).

Pathological conditions, such as fetal hypoxia and growth retardation, appear to be associated with an increase in systemic and placental vascular resistance, respectively (Cohn et al., 1985; Yaffe et al., 1987; Creasy et al., 1973; Clapp et $a l ., 1980)$. An increase in tissue vascular resistance is, apart from a reduction of blood flow to that tissue, often associated with an increase in mean arterial pressure. Since both the systemic and placental circulations derive from the fetall aorta, an increase in vascular resistance in one of these circulations, without a decrease in the other, may result in an increase in mean arterial pressure.

On the basis of the positive association between systemic vascular resistance and aorta PI during fetal development on the one hand, and the positive association between systemic or placental vascular resistance and mean arterial pressure on the other hand, it is suggested that an increase in mean arterial pressure is associated with an increase in aorta PI. It was hypothesized that an acute increase in fetal mean arterial pressure, as induced by norepinephrine and angiotensin II (Berman et al., 1978), would result in an increase in aorta PI above the level of the major abdominal arteries. A close correlation between 
aorta PI and mean arterial pressure, might provide a tool for noninvasive assessment of fetall blood pressure. We studied the correlation and regression between the aorta PI and mean arterial pressure, the latter being acutely altered by norepinephrine and angiotensin $\mathrm{II}_{\mathrm{s}}$ in eight anesthesized fetal sheep.

\section{MATERIAL AND METHODS}

Surgery. Surgery was performed on eight pregnant sheep of the Dutch Texel breed between the 112th and 127th day of gestation (term 147 days). Anesthesia was induced with pentobarbital infused intravenously and was maintained with $1 \%$ halothane in a 2:1 mixture of nitrous oxide and oxygen. After opening of the matemal abdomen through a paramedian incision, the uterus was incised over the fetal pelvis and the fetal lower body was exteriorized. The fetal aorta was approached retroperitoneally. A $20 \mathrm{MHz}$ pulsed Doppler transducer was mounted on the traject between the diaphragm and the origin of the coeliac trunk. Subsequently, an electromagnetic flow meter with an inner diameter of 4 or 5 $\mathrm{mm}$ (Skalar, Delft, The Netherlands) was applied around the common umbilical artery. Immediately next to the electromagnetic flow meter and on the common part of the umbilical artery as well, we mounted a $20 \mathrm{MHz}$ pulsed Doppler transducer. A polyvinyl catheter (outer diameter $1.6 \mathrm{~mm}$, inner diameter 0.8 $\mathrm{mm}$ ) was inserted into one of the femoral arteries and advanced up into the abdominal aorta. Another polyvinyl catheter was inserted via the femoral vein into the inferior vena cava. For another experimental protocol, a cotyledonary artery and vein were cannulated and the catheters were advanced up to an umbilical artery and vein, respectively. The uterus was closed in one layer, while the incision in the maternal abdominal wall, through which the catheters, Doppler and flow leads were exteriorized, was approximated using surgical clamps. The surgical and experimental procedures were approved by the local Animals Medical Ethics Committee.

Experiments. The experiments started one hour after completion of the surgical procedure, while the ewe was in right lateral tilt position and general anesthesia was maintained. In a random order, angiotensin II ( $5 \mathrm{microgram} / \mathrm{ml}$ ) and norepinephrine $(10 \mathrm{microgram} / \mathrm{ml})$ were administered once or twice in boluses of $1 \mathrm{ml}$ via the catheter in the inferior vena cava. In three fetal sheep, one of the bolus injections with norepinephrine, was replaced by a norepinephrine infusion at a rate of $10 \mathrm{microgram} / \mathrm{min}$ during 5 minutes. Each drug administration was followed by at least a 30 -minutes recovery period. Measurements included fetal mean arterial pressure and fetal heart rate, on-line derived from the arterial pressure pulse using a tachometer. The pressure signal was 
determined with the zero point at the level of the ewe's abdomen. Both arterial pressure and heart rate were fed into amplifiers (Hewlett Packard 8800 Series, Hewlett Packard Company, Andover, Massachusetts) „displayed on monitor, recorded on strip-chart recorder and stored on magnetic tape. Blood flow velocity waveforms in the subdiaphragmatical aorta could be obtained by means of the implanted $20 \mathrm{MHz}$ pulsed Doppler transducer in 7 fetuses (the transducer hampered in 1 fetus), while in 4 fetuses, signals could be obtained from the external iliac artery by means of the Doppler transducer on the common umbilical artery. Adjustment of the depth resolution resulted in completely separate signals from the common umbilical and external iliac artery, respectively. The frequency shifted signals were stored in quadrature on audiotape and processed by a spectrum analyser (Doptek Ltd., Chicester, England). The PI, as used in our experiments for waveform description, was defined as the difference between the maximum and minimum frequency shift divided by the mean frequency shift of the maximum envelope curve throughout the cardiac cycle. The waveform index calculations were performed off-line using a handguided light-pen (Ruissen et al., 1987a).

Calculations and data analysis. All variables were simultaneously recorded for further analysis. If an experiment was performed twice in one fetus, the mean value of both experiments was used for paired comparison of the experimental with the baseline value. The paired comparisons were not subjected to statistical analysis because of the small number of fetuses involved in either angiotensin II administration $(n=4)$ and norepinephrine infusion $(n=3)$. It is resumed that the aim of this study was to evaluate the relationship between the aorta PI and mean arterial pressure in a condition of fetal hypertension. To this end, a linear regression equation was fitted to the paired observations of the abdominal aorta PI and mean arterial blood pressure, and the correlation coefficient between both variables, calculated. These descriptive statistical parameters were also calculated for the relationship between the external iliac artery PI and mean arterial pressure. All data obtained during baseline and experimental conditions were included in the analysis, irrespective of the sort of drug administered and the period of time passed by after administration. The restrictions were that fetal heart rate was within the normal range $( \pm 2 S D)$ at the time of measurement. As a consequence, the experimental values were obtained between 2 and 3 minutes after norepinephrine or angiotensin II bolus administration and during norepinephrine infusion.

Fetal heart rate and mean arterial pressure were calculated over 10 -seconds periods using a personal computer program. The PI values in the aorta and external iliac artery were calculated from five consecutive cardiac cycles within the same, 10-seconds period. In the fetal aorta, we also measured the period of 
time $(T)$ between the onset of blood flow velocity acceleration at the beginning of a cardiac cycle and the first dicrotic notch (fdn) in the diastolic part of the waveform. This notch has been shown to occur in response to peripheral wave reflections (Burns, 1988b). This parameter was defined as Tfon. The values of $T_{\text {fdn }}$ were calculated from the same waveforms as from which the PI values were derived. The relationship between $T_{\text {fdn }}$ and mean arterial pressure was also assessed by regression and correlation analysis. In addition, the limits of the 95\% confidence interval for prediction of $T_{\text {fdn }}$ on the basis of random values for mean arterial pressure were calculated.

\section{RESULTS}

Bolus administration of norepinephrine resulted in an acute acceleration of fetal heart rate and an increase in mean arterial pressure in the first 10 seconds after injection. Subsequently, three different patterns of heart rate response were observed. Firstly, a reflex-bradycardia on the basis of baroreceptor activation, which lasted for approximately 15-30 seconds and then again acutely changed into a tachycardia, which slowly decelerated to baseline level over a period of 2 minutes. Secondly, a less deep reflex-bradycardia, which gradually returned to baseline level within 3 minutes, and thirdly, a tachycardia which decelerated slowly and reached a level below baseline fetal heart rate after approximately 5 minutes and then, after a steady acceleration retumed to baseline levels within 10 minutes. These inconsistent cardiovascular responses probably proceed from altered autonomic reflex mechanisms during anesthesia (Assali et al., 1974).

In all experiments, fetal blood pressure was maximal in the first 2 minutes after administration, then slowly decreased, and was nomal again 5 minutes after administration.

Infusion of norepinephrine resulted in a sustained tachycardia and hypertension as long as norepinephrine was administered, or in a reflex-bradycardia after an initial, short-lasting tachycardia. After approximately 5 minutes, when infusion was ended, this reflex-bradycardia turned again into a tachycardia, which vanished after more than 10 minutes.

Bolus administration of angiotensin II resulted in tachycardia after 1 minute, mild tachycardia, normal fetal heart rate or mild bradycardia between 2 and 4 minutes, whereafter fetal heart rate returned to normal levels within 10 minutes after administration. Fetal mean arterial pressure was maximal after 2 minutes and normal again after approximately 5 minutes. 
Table 8.1 lists the mean values and standard deviations of the variables under investigation. Measurements in the fetal aorta were performed in 7 fetuses after norepinephrine bolus administration, in 4 fetuses after angiotensin II bolus administration and in 3 fetuses during norepinephrine infusion. Measurements in the external iliac antery were performed in 2 fetuses after norepinephrine bolus administration and in 4 fetuses after angiotensin II bolus administration. The number of observations per fetus varied between 3 and 13, and was 8 on average.

Figure 8.1, upper panel, shows the relationship between the external iliac artery PI and mean arterial pressure as derived from four anesthesized fetal lambs in this study. The slope of the linear regression equation fitted to these data is 0.082 and the coefficient of correlation 0.72 .

Table 8.1. Mean variable values before and after drug administration. The baseline values were obtained 1 minute prior to drug administration whereas the experimental values were obtained 2-3 minutes after bolus administration or $1-5$ minutes after start of infusion.

\begin{tabular}{|c|c|c|c|c|c|c|}
\hline \multirow[t]{3}{*}{ variables } & \multicolumn{2}{|c|}{ norepineplarine bolus adm. } & \multicolumn{2}{|c|}{ angiotensin II bolus adm. } & \multicolumn{2}{|c|}{ norepinephrine infusion } \\
\hline & baseline & experiment & baseline & experiment & baseline & experiment \\
\hline & \multicolumn{2}{|c|}{$\mathrm{n}=7$ fetuses } & \multicolumn{2}{|c|}{$\mathrm{n}=4$ fetuses } & \multicolumn{2}{|c|}{$\mathrm{n}=3$ fetuses } \\
\hline $\begin{array}{r}\text { mean arterial pressure } \\
\qquad(\mathrm{mmHg})\end{array}$ & $33.4 \pm 3.6$ & $48.9 \pm 5.9$ & $31.9 \pm 3.5$ & $43.9 \pm 6.5$ & $33.0 \pm 4.6$ & $53.7 \pm 4.9$ \\
\hline abdominal aorta PI & $1.69 \pm 0.12$ & $1.97 \pm 0.30$ & $1.66 \pm 0.11$ & $2.24 \pm 0.20$ & $1.73 \pm 0.18$ & $2.27 \pm 0.49$ \\
\hline$T_{f d n}(\mathrm{~ms})$ & $1511 \pm 18$ & $95 \pm 25$ & $130 \pm 7$ & $92 \pm 14$ & $133 \pm 7$ & $79 \pm 9$ \\
\hline Petal hears rate (bpm) & $179 \pm 18$ & $173 \pm 35$ & $174 \pm 17$ & $184 \pm 27$ & $195 \pm 10$ & $206 \pm 43$ \\
\hline \multirow{2}{*}{$\begin{array}{l}\text { umbilical blood flow } \\
\qquad(\mathrm{ml} / \mathrm{min})\end{array}$} & $329 \pm 1.42$ & $387 \pm 183$ & $322 \pm 144$ & $252 \pm 144$ & $385 \pm 185$ & $485 \pm 316$ \\
\hline & \multicolumn{2}{|c|}{$n=2$ fetuses } & \multicolumn{2}{|c|}{$n=4$ fetuses } & & \\
\hline $\begin{array}{l}\text { mean arterial pressure } \\
\text { (mmHg) }\end{array}$ & $37.0 \pm 0.4$ & $52.9 \pm 3.6$ & $34.4 \pm 3.1$ & $46.4 \pm 4.3$ & & \\
\hline external iliac artery PI & $2.22 \pm 0.36$ & $3.97 \pm 0.86$ & $1.86 \pm 0.58$ & $2.72 \pm 0.32$ & & \\
\hline fetal heat rate (bpm) & $166 \pm 1$ & $161 \pm 13$ & $180 \pm 26$ & $185 \pm 19$ & & \\
\hline
\end{tabular}



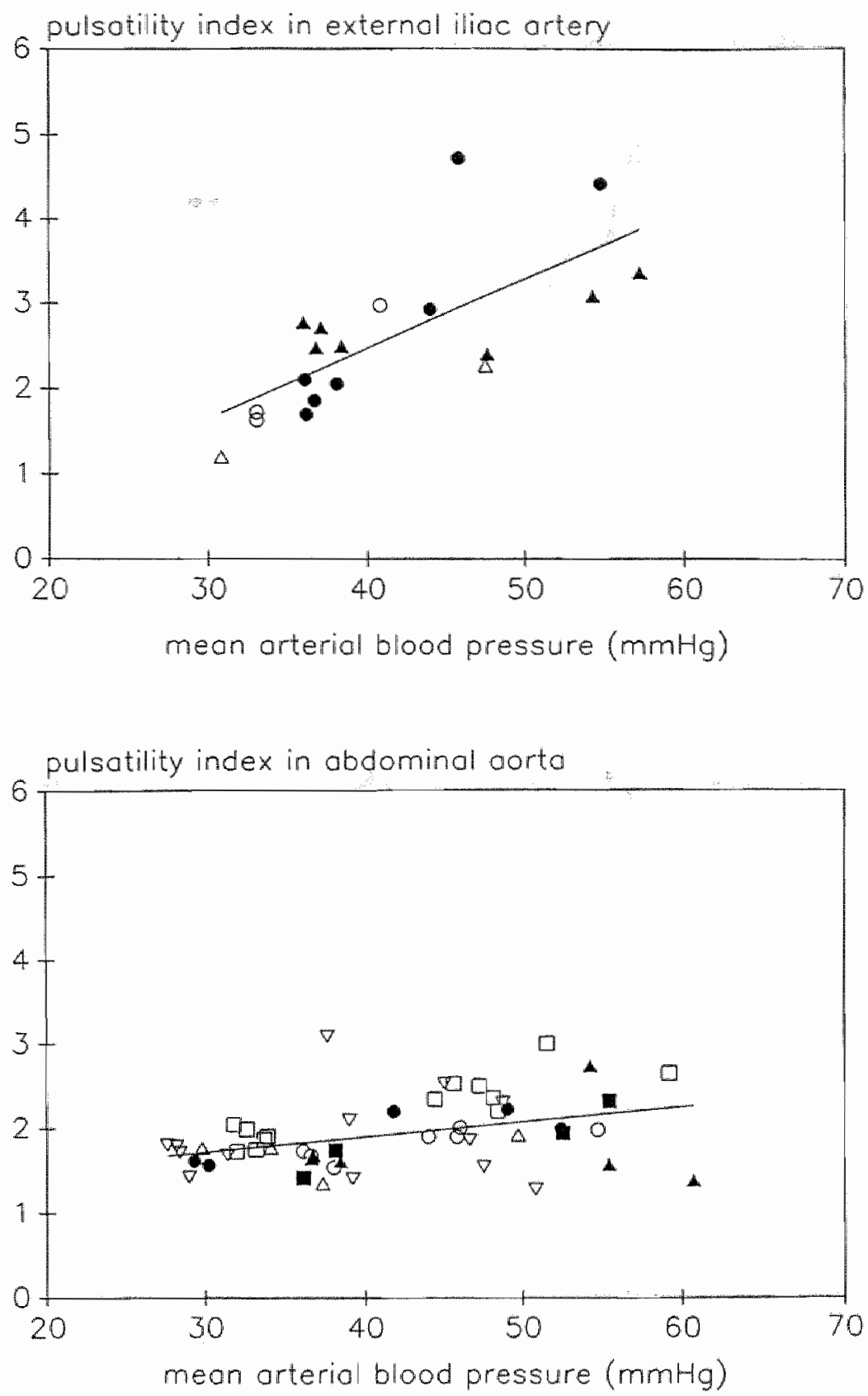

Figure 8.1. Upper panel: observations of external iliac artery PI and fetal mean arterial pressure in 4 anesthesized fetal lambs prior and secondary to norepinephrine and angiotensin II administrations. Lower panel: observations of abdominal aorta PI and fetal mean arterial pressure in 7 anesthesized fetal lambs prior and secondary to norepinephrine and angiotensin II administrations. 

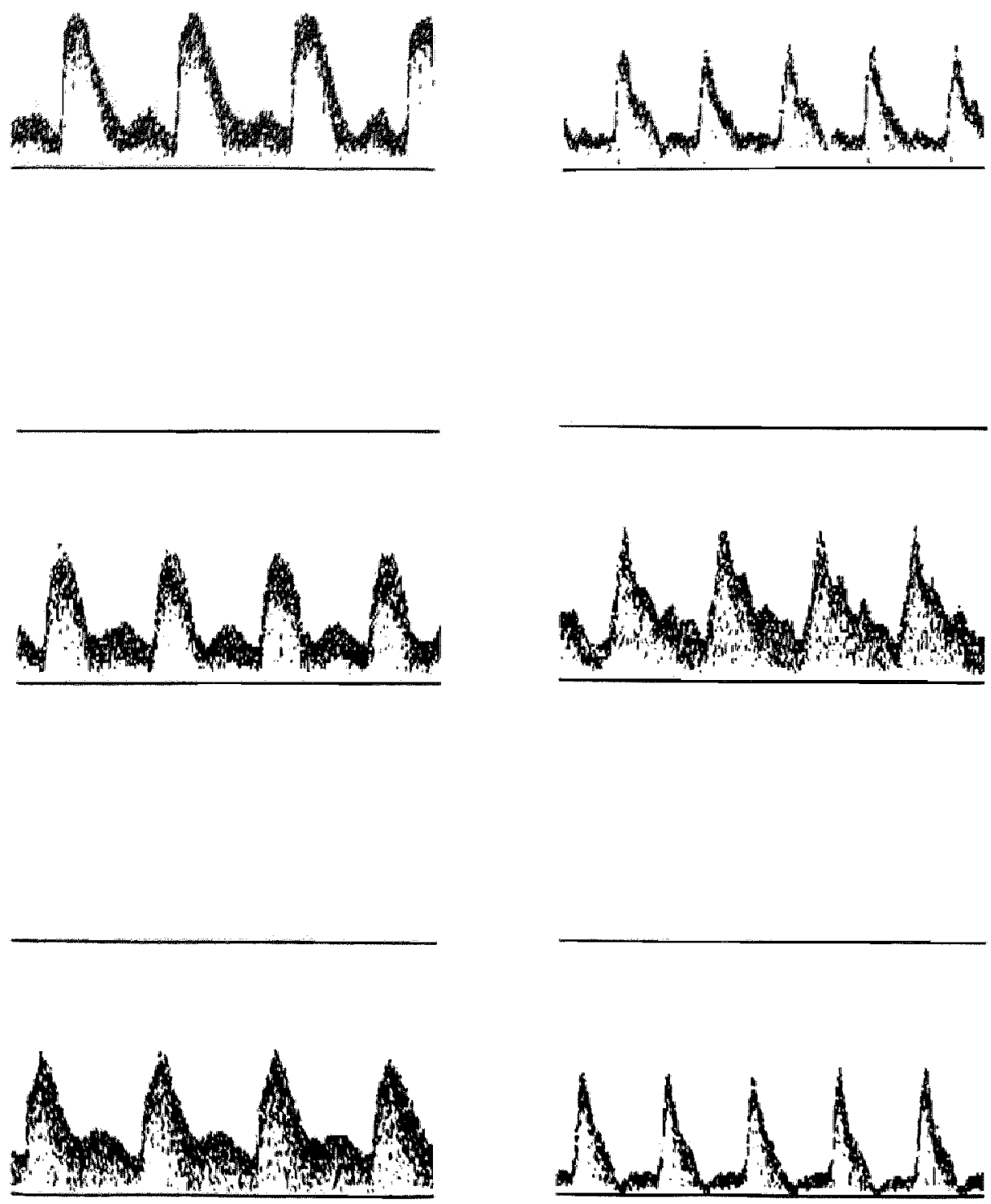

Figure 8.2. Upper panel: blood flow velocity waveforms in the abdominal aorta during baseline condition (left) and secondary to norepinephrine bolus administration (right). Middle panel: blood flow velocity waveforms in the abdominal aorta during baseline condition (left) and secondary to norepinephrine infusion (right). Lower panel: blood flow velocity waveforms in the external iliac artery during baseline condition (left) and secondary to angiotensin II bolus administration (right). 
Figure 8.1, lower panel, shows the relationship between the subdiaphragmatical abdominal aorta PI and mean arterial pressure as derived from seven fetal lambs. The slope of the linear regression equation is 0.018 and the coefficient of correlation 0.40 .

Figure 8.2 shows the waveform changes in the aorta of one of the fetal lambs prior to (left panel) and after (right panel) norepinephrine bolus administration. In this experiment, an increase in mean arterial pressure from 34.1 to 49.7 $\mathrm{mmHg}$ was associated with an increase in aorta PI from 1.77 to 1.92 . It was noted that the changes observed in the aortic blood flow velocity waveform during an increase in blood pressure, did often not concur with numerical PI changes. Figure 8.2, middle panel, shows the waveform changes in the aorta of another fetal lamb before and during norepinephrine infusion. In this experiment, an increase in mean arterial pressure from 29.0 to $50.8 \mathrm{mmHg}$ was associated with a decrease in aorta PI from 1.43 to 1.28. The waveforms during hypertension (right) were characterized by an undulant pattern of the diastolic blood flow velocity. In the waveform obtained during baseline condition, the first acceleration of blood flow velocity during diastole occurred after 134 milliseconds. In contrast, the first diastolic acceleration during hypertension accurred after 88 milliseconds. In the example displayed in the upper panel of figure 8.2, the differences were even larger, 195 milliseconds at baseline condition versus 68 milliseconds during hypertension. Roughly, fetal hypertension appeared to be associated with a narrowing of the systolic peak and a shortening of the $T_{\mathrm{fdn}}$. Figure 8.3 shows the relationship between the $\mathrm{T}_{\mathrm{fdn}}$ in the subdiaphragmatical abdominal aorta and mean arterial pressure as derived from seven fetal lambs. The slope of the linear regression equation was -2.67 and the coefficient of correlation -0.77 . The lines in the figure represent the linear regression line and the upper and lower limits of the $95 \%$ confidence interval with regard to prediction of the $T_{\text {fdn }}$ in the subdiaphragmatical aorta on the basis of a random value of fetal mean arterial pressure (Johnson, 1984). In an inverse condition, this interval might have implications for prediction of mean arterial pressure on the basis of the $\mathrm{T}_{\text {fdn }}$.

Figure 8.2, lower panel, shows an example of the waveform changes in the external iliac artery secondary to the administration of angiotensin II. The nature of the changes was in correspondence with those observed in the fetal aorta, but the expression was more pronounced. Both reduction of diastolic blood flow velocity and narrowing of the systolic peak were frequently observed together and sometimes resulted in a minor, in other cases in a major increase in PI value during fetal hypertension. Nevertheless, extemal iliac artery PI did not unambiguously vary with mean arterial pressure, but seemed, as the abdominal aorta PI, also to be affected by other factors. 


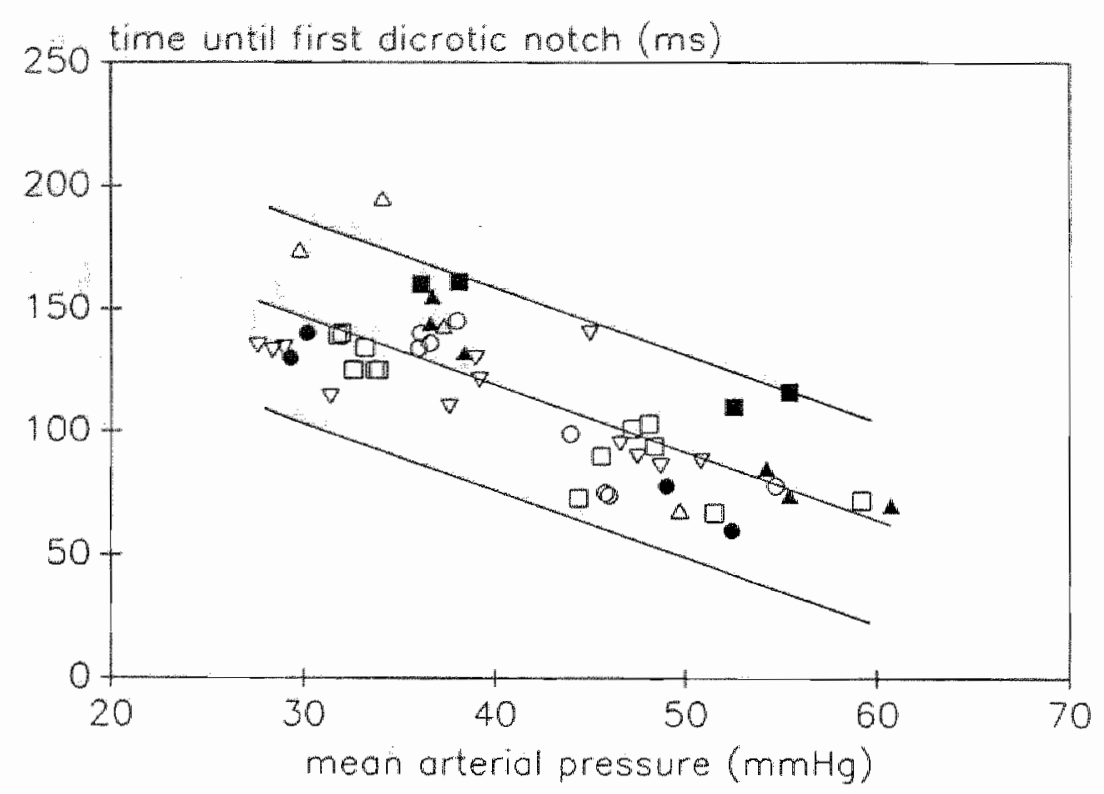

Figure 8.3. Observations of $T_{\text {fdu }}$ in the abdominal aorta blood flow velocity waveform and mean arterial pressure in 7 anesthesized fetal lambs prior and secondary to norepinephrine and angiotensin II administrations. The lines represent the linear regression line and the $95 \%$ prediction interval between Tfdn and mean arterial pressure.

\section{DISCUSSION}

It is concluded from this study that administration of hypertensive drugs results in changes of the blood flow velocity waveforms in the fetal systemic circulation. However, these changes were not consistent with regard to their nature and degree of expression. In response to norepinephrine, the abdominal aorta PI considerably increased in conditions that mean arterial pressure was again returning to baseline levels and slightly decreased in conditions that mean arterial pressure had nearly doubled. A decrease in abdominal aorta PI particularly developed in the first minute of norepinephrine infusion and was associated with increased umbilical blood flow, the latter possibly due to an increase in cardiac contractility. In the next minutes of norepinephrine infusion, when blood pressure and umbilical blood flow slightly decreased but were still at an increased level, the abdominal aorta PI concurrently increased on the basis of a narrowing of the systolic peak and a reduction of the diastolic velocities.

Following norepinephrine and angiotensin II bolus administration, waveform changes were preferably studied in a condition of maximum hypertension and steady fetal heart rate. This condition was generally reached between 2 and 3 
minutes after injection, for both norepinephrine and angiotensin II. As shown in table 1, the mean increase in aorta PI was higher in response to angiotensin II, whereas the increase in mean arterial pressure was higher in response to norepinephrine. This result might be explained by the mechanism of action of angiotensin II. Angiotensin II probably constricts the umbilical arteries (Adamson et al., 1989a) and thus induces wave reflections at a proximal level of the circulation (Burns, 1988a). The wave reflections from the umbilical circulation might contribute to the changes observed in aorta PI. The smaller increase in mean arterial pressure in response to angiotensin II might be explained by a dose-related smaller amount of systemic vasoconstriction.

It has been shown that angiotensin II raises umbilical arterial vascular resistance, whereas norepinephrine predominantly raises umbilical venous vascular resistance (Adamson et al, 1989a). In correspondence, we observed a decrease in umbilical arterial blood flow in response to angiotensin II and an increase in response to norepinephrine, the latter probably being due to increased distribution of blood flow towards the placenta (Berman et al., 1978). The combination of systemic vasoconstriction and increased umbilical blood flow might be associated with either an increase or a decrease in blood flow through the descending aorta. It is therefore suggested that, in most physiological conditions, a significant increase in PI is primarily associated with a substantial decrease in blood flow. Norepinephrine as well as angiotensin II presumably induced a substantial decrease in blood flow to the lower leg and thus a consistent increase in external iliac artery PI.

Although acute systemic hypertension was not associated with marked changes in the abdominal aorta PI, it was possible to discern a change in another dynamic characteristic of the blood flow velocity waveform, being the time course between the onset of the cardiac cycle and the occurrence of a first dicrotic notch. Such an incisural notch is likely to be the result of pulse wave reflection from the peripheral circulation. Investigations have shown that arterioles contribute most to arterial pulse wave reflections (O'Rourke, 1982). If vasoconstriction occurs, the pulsatility of the velocity pulse upstream increases. In addition, there is a brief, abrupt upswing in pressure during the deceleration phase of systole, forming a notch in the pressure and velocity waveforms. The small undulations following the notch are known as the dicrotic wave and can be traced to reflections from the distal arterial tree (Burns, 1988a). In normotensive fetuses, the first (visible) dicrotic notch appeared to coincide with the diastolic acceleration of the blood flow velocity, which results from aortic and pulmonary valve closure (Griffin et al., 1984). In hypertensive fetuses however, notching already occurred in the descending slope of the systolic peak, indicating increased impedance to blood flow (Burns, 1988a). Jouppila \& Kirkinen 
(1984) described absence of end-diastolic blood flow velocity in the thoracic aorta of nine human fetuses with signs of fetal distress. Visual examination of the exemplary time-velocity spectra presented in their report, mainly suggest a narrowing of the systolic peak. In another report by the same authors (Jouppila \& Kirkinen, 1986), the duration of the systolic peak in the thoracic aorta of distressed fetuses again seemed to be less than that of unstressed fetuses. This narrowing of the systolic peak appears to be associated with a slight decrease in Tfan. Computer modeling of the uteroplacental circulation has shown that both an increase in waveform PI and occurrence of a dicrotic notch result from an increase in vascular resistance and not from changes in mean arterial pressure (Adamson et al., 1989b). This provides additional support for the concept that dicrotic notching results from peripheral wave reflections and might thus be more useful for assessment of peripheral vascular resistance than for assessment of arterial blood pressure.

Unfortunately, our data do not provide information on the relevance of fetal aorta blood flow velocity waveform assessment with regard to changes in fetal mean arterial pressure which occur as adaptation mechanisms in response to potential stress factors in human fetuses. The increase in mean arterial pressure during fetall hypoxia does probably not exceed 10-20 percent of baseline arterial pressure in normally developed (Parer, 1980; Muijsers et al., 1990a) as well as in growth-retarded fetuses (Block et al., 1984), whereas the increase in arterial pressure in fetal sheep with embolized placentas remains within the same range (Morrow et al., 1989; Muijsers et al., 1990b). It is suggested that changes in parameters derived from the aorta blood flow velocity waveform during fetal stress will not exceed the range of normal variation. For proper evaluation of the relationship between fetal arterial blood pressure and parameters derived from the aorta blood flow velocity waveform, it would be advisable to study both variables under physiological stress conditions, such as hypoxia and/or as a function of gestational age, in unanesthetized fetuses. 


\section{CHAPTER 9}

\section{General discussion and conclusions}

The objective of this study was to address various questions raised with regard to the rationale of Doppler velocimetry in obstetrics. It was pursued to address some basic questions from a patho-physiological point of view, using the fetal lamb in utero as an experimental model. Results obtained from experiments in fetal sheep are not, without due consideration, transferable to the undisturbed human fetus. Factors such as species characteristics and surgery may play an important role, while anesthesia, as used in some experiments, may also affect cardiovascular reflex responses. General statements and conclusions with regard to human pregnancy, should therefore be regarded as a tentative, and not as a final judgment.

A first objective was to evaluate the possible role of Doppler blood flow velocimetry as a diagnostic test for fetal hypoxemia. Physiological studies have demonstrated that the fetus responds to hypoxemia with a series of circulatory changes, based on regional changes in organ vascular resistance. These changes include a redistribution of blood flow in favor of heart, brain and adrenals, but at the expense of skin, muscles, kidneys, intestines and lungs. Total cardiac output remains relatively stable as well as cardiac output distribution to the placenta. It has further been shown that, during hypoxemia, vascular resistance between abdominal aorta and cotyledonary vein remains relatively constant (Paulick et al., 1990). The latter finding suggests that the umbilical arteries and fetal placental capillaries do not actively constrict during hypoxemia.

Changes in organ vascular resistance induce changes in the Doppler blood flow velocity waveform in the artery that supplies that organ. In obstetrics, changes in blood flow velocity waveforms are generally quantified by dimensionless waveform indices. In this study the Pulsatility Index (PI) was used on the basis of its superiority in waveform description (Ruissen et al., 1987a). An 
increase in the PI (or S/D-ratio) of a blood flow velocity waveform implies a relative reduction, absence or reversal of diastolic blood flow velocity within the artery under investigation.

It was hypothesized that, in conditions without active interference with the umbilical circulation such as umbilical cord occlusions (van Vugt et al., 1988a), fetal hypoxemia would not result in major changes in the umbilical artery PI. The relationship between the umbilical artery PI and fetal oxygenation was studied in response to three different mechanisms of reduced oxygen delivery to the fetus. These mechanisms included:

- a gradual reduction of uteroplacental blood flow by means of progressive matemal common internal iliac artery occlusion;

- administration of low oxygen gas mixtures to the maternal sheep;

- embolization of the uteroplacental microcirculation using non-radioactive microspheres.

None of these mechanisms resulted in a significant change in umbilical artery PI, neither acutely, within several hours, nor chronically, after several days. Even in individual fetal sheep, changes of any significance were not observed. It is concluded from these studies that severe fetal hypoxemia may exist, without any change in the waveform of the umbilical artery blood flow velocity.

Morrow et al. (1989) demonstrated that embolization of the fetoplacental circulation resulted in an increase in the umbilical artery S/D-ratio and in a decrease in fetal oxygenation. In this experiment, fetal hypoxemia probably resulted from a decrease in umbilical blood flow. In another experiment, Morrow et al. (1990) examined the response of the umbilical artery waveform and calculated umbilical vascular resistance to fetal hypoxemia, induced by reduction of maternally inspired oxygen. No changes were observed in both variables, which fully agrees with the results of our experiments.

Integration of our own results with those of Morrow et al. (1989), suggests that the umbilical artery blood flow velocity waveform only identifies an increase in umbilical vascular resistance and not particularly fetal hypoxemia. In the experiments by Morrow et al. (1989), a major change in the umbilical artery waveform appeared to be associated with only a moderate decrease in fetal oxygenation. It is therefore suggested that fetuses with abnormal umbilical artery waveforms are prone to develop, but not necessarily suffer from hypoxemia. This suggestion agrees with results from Reuwer et al. (1987) in human fetuses, that absence of umbilical blood flow in diastole may precede cardiotocographic signs of fetal distress by several weeks or even months. In addition, data from Ferrazzi et al. (1988) show that fetuses with a normal and an 
abnormal umbilical artery $\mathrm{PI}$, respectively, may have identical arterial oxygen content values. In contrast, Weiner (1990) considers every fetus to be hypoxemic by the time diastolic flow in the umbilical artery is lost and advises cordocentesis to prove absence of fetal acidosis. In conclusion, fetuses with abnormal waveforms are prone to develop hypoxemia on the basis of progressive impairment of placental gas exchange (Morrow et al, 1989; Weiner, 1990 ), but on the other hand, a normal umbilical artery waveform cannot be considered as a sign of normoxemia.

In human fetuses, relative reduction of diastolic blood flow velocity in the umbilical artery correlates with the number of abnormal small arteries in the fetal placental villi (Giles et al., 1985; Fok et al., 1990). It was suggested that abnormality of these small arteries provide an anatomical basis for elevated resistance to blood flow in placentas from growth-retarded fetuses. In order to evaluate the relationship between the umbilical artery PI and umbilical vascular resistance, a study was designed in which the umbilical microcirculation was selectively embolized via a catheter in a major cotyledonary artery. Impedance to umbilical blood flow as assessed by the PI and umbilical vascular resistance as calculated from umbilical blood flow and pressure gradient were both determined from measurements at the level of the common umbilical artery. Thus, it was possible to compare total change in PI with total change in umbilical blood flow and umbilical vascular resistance, respectively. A strong correlation could be demonstrated between umbilical artery PI and umbilical vascular resistance. The regression slopes between both variables were comparable for all fetuses $(n=8)$ studied. However, whether the increase in PI mainly results from an increase in resistance or also from a decrease in compliance remains unknown from these data. If chronic fetal hypoxia results in an obliteration of the small arteries in the tertiary stem villi (Giles et al., 1985) and simultaneously in an increase in the number of short capillaries (Scheffen et al., 1989), it is suggested that placental compliance does not decrease and that the increase in PI mainly results from an increase in placental vascular resistance. The steepness of the regression slopes between the umbilical artery PI and umbilical blood flow varied among the fetuses, but the PI tended to increase abruptly when a critical level of umbilical blood flow was reached. At that critical level of umbilical blood flow, the fetus appeared to fail in further increasing its blood pressure.

Absence or reversal of end-diastolic blood flow in the umbilical circulation represents a riskful condition with minute fetal placental circulatory reserve capacity (Reuwer et al., 1986a). 
Whether avallability of umbilical artery Doppler blood flow velocimetry improves obstetric management and outcome cannot be concluded from experimental data, but requires clinical studies.

If management would be performed on the basis of a single umbilical Doppler measurement, then the availability of this test would inevitably have adverse effects. In three fetal sheep, cotyledonary embolization resulted in conditions in which end-diastolic flow was absent in the artery which directly supplied the embolized region, whereas the waveforms in the fetal aorta or common umbilical artery were unaffected, suggesting an adequate overall fetal placental circulatory reserve capacity. It has been shown in case reports on human pregnancy that normal and abnormal umbilical artery waveforms may exist together in one fetus (Trudinger \& Cook, 1988; Harper \& Murnaghan, 1989). It was concluded that, in the case of discordant waveforms, delivery may be safely delayed and that premature intervention, without further signs of fetal distress, should be prevented.

On the basis of active changes in vascular resistance in different vascular beds during hypoxemia, it is suggested that Doppler waveform measurements might provide noninvasive information on blood flow distribution in the fetus and consequently, on the actual state of fetal oxygenation. Wladimiroff et al. (1986) studied the blood flow velocity waveform in the internal carotid artery of growth-retarded human fetuses, and found a relative increase in diastolic blood flow velocity, suggesting a compensatory reduction in fetal cerebral vascular resistance and thus a brain-sparing effect in the presence of fetal hypoxia. In fetal sheep, Doppler study of the carotid artery is insensible on the basis of circulatory differences and the relatively small amount of cerebral tissue present. To examine the relevance of fetal Doppler studies in aid of identification of fetal hypoxemia, we studied the femoral artery waveforms for a number of reasons:

- the resistance in this vascular bed during hypoxemia might increase by 300 percent (Yaffe et al., 1987);

- the femoral artery supplies a major part of both the ovine and human fetal body and enables comparisons between both species;

- the femoral artery can easily be approached for placement of a Doppler transducer and does not require major surgical fetal disturbance.

Fetal hypoxemia, induced by a gradual reduction of uteroplacental blood flow by means of progressive maternal common internal iliac artery occlusion in four fetal lambs, was associated with an increase in the femoral artery PI in each of these fetuses. However, the amount of change differed per fetus, either as a result of short periods of circulatory adaptation, or because of an actual 
wide variability in possible waveform characteristics. Therefore, the femoral artery blood flow velocity waveform was also studied during hypoxemia, induced by administration of low oxygen gas mixtures to the maternal sheep. In this study, the fetus was allowed to adapt to major changes in oxygenation for one hour, and the femoral artery PI was studied as a ratio with the umbilical artery PI. Thus, it was meant to develop a parameter, more sensitive to changes in blood flow distribution and less sensitive to changes in other physiological variables, such as fetal heart rate (Mulders et al., 1986). In this study, a somewhat less consistent pattern was observed with regard to the amount of change in femoral artery PI. It appeared that the femoral artery PI only increased in association with an increase in mean arterial pressure and that a strong correlation existed between both variables. Since both the femoral artery PI and mean arterial pressure correlate with peripheral vascular resistance, it remains unexplained why a presumed vasoconstriction did not occur in two fetuses, and occurred in various degrees in the major part of the sample group $(n=8)$. A possible explanation is the presence of hypercarbia in the experiment in which uteroplacental blood flow was reduced. Hypercarbia might have resulted in an enhanced chemoreceptor activation (Dawes et al., 1968; Blanco et al., 1984), and thus a more pronounced musculocutaneous vasoconstriction.

Several investigators studied the correlation between Doppler waveform measurements in the peripheral circulation and the degree of oxygenation in the human fetus, obtained by cordocentesis. Vyas et al. (1989) studied the relationship between the $\mathrm{PaO}_{2}$ in the umbilical artery or vein and the renal artery PI in 18 growth-retarded fetuses and found a linear regression with a correlation coefficient of -0.66 . Bilardo et al. (1990), studied the relationship between a mathematical index derived from Doppler measurements un the common carotid. artery and the aorta and an "asphyxia" index derived from blood gases and pH in the umbilical vein. They found a linear regression with a correlation coefficient of -0.67 and suggested that Doppler measurements can be used to establish the probability of a given fetus being hypoxemic and acidemic. However, it should be focused that with a correlation of -0.67 , only 45 percent of the fetuses assessed by Doppler is encompassed by the 95 percent confidence interval of the calculated regression line for asphyxia. Conversely, in 55 percent of the fetuses assessed by Doppler, the prediction of blood gases and $\mathrm{pH}$ does not correspond with the value predicted by the regression line. It is concluded that the human fetus similarly as the fetal lamb, responds to hypoxemia with circulatory changes. However, it seems not feasible that Doppler measurements in peripheral fetal vessels can be used for noninvasive assessment of fetal oxygenation. 
More feasible seems the assumption that Doppler measurements in the systemic circulation provides sensible information on hemodynamic characteristics of the fetal circulation. On the basis of the finding that the femoral artery PI correlates with fetal mean arterial pressure during hypoxemia, it was hypothesized that the same concept would be valid for the fetal aorta, at the level above the visceral organs. Several investigators already focused on Doppler measurements in the fetal aorta as an actual reflection of active circulatory regulation mechanisms (Griffin et al., 1984; Jouppila \& Kirkinen, 1986; Tonge et al., 1986; Hackett et al., 1987). Furthermore, the performance of Doppler measurements in the fetal aorta is easier than in small peripheral arteries. We examined the relationship between the aorta PI and mean arterial pressure, the latter being acutely changed by administration of norepinephrine and angiotensin II. We found a rather weak correlation, not feasible for noninvasive assessment of fetal arterial blood pressure.

In summary, blood flow velocity waveforms of fetal arteries reflect the impedance or resistance to blood flow at the site of measurement, but constituted distally from the site of measurement. Various anatomical and pathological stress conditions can account for a change in vascular resistance in an organ and thus, in a change in the blood flow velocity waveform of the artery which supplies that organ. Since blood flow velocity waveforms can be obtained from various fetal arteries, each waveform has its own interpretation, but roughly, they all reflect a change in organ vascular resistance, which in practice often implies opposite changes in organ blood flow and fetal systemic arterial blood pressure. Blood flow velocity waveforms from both the umbilical and fetal systemic arteries do not provide reliable information on the virtual fetal condition. However, Doppler measurements may identify fetuses at increased risk for adverse neonatal outcome more effectively than current methods of fetal surveillance. This hypothesis can only be answered in clinical studies, evaluating the availability of Doppler ultrasound on obstetric management and outcome. 


\section{References}

Adamson SL, Morrow RJ, Bull SB, Langille BL (1989a) Vasomotor tesponses of the umbilical circulation in fetal sheep. Am J Physiol 256:1056-1062.

Adamson SL, Morrow RJ, Bascom PAJ, Mo LYL, Ritchie JWK (1989b) Effect of placental resistance, arterial diameter, and blood pressure on the uterine arterial velocity waveform: a computer modeling approach. Ultrasound Med Biol 15:437-442.

Anderson DF, Bissonnette JM, Faber JJ, Thornburg KL (1981) Central shunt flows and pressures in the mature fetal lamb. Am J Physiol 241:H60-H66.

Assali NS, Brinkman III CR, Nuwayhid B (1974) Comparison of maternal and fetal cardiovascular functions in acute and chronic experiments in the sheep. Am J Obstet Gynecol $120: 411-425$.

Assali NS, Brinkman III CR, Woods JR, Dandavino A, Nuwayhid B (1978) Development of neurohumoral control of fetal, neonatal and aduit cardiovascular functions. Am J Obstet Gynecoll 129:748-759.

Barcroft J (1946) Researches on pre-natal life. Blackwell, Oxford, 1946.

Barcroft J \& Barron DH (1946) Observations upon the form and relations of the matemal and fetal vessels in the placenta of the sheep. Anat Rec 94:569-595.

Barrett CT, Heymann MA, Rudolph AM (1972) Alpha and beta adrenergic receptor activity in fetal sheep. Am J Obstet Gynecol 112:1114-1121.

Baskett TF, Allen AC, Gray JH, Young DC, Young LM (1984) Antepartum fetal assessment using a fetal biophysical profile score. Am J Obstet Gynecol 148:630-633.

Baskett TF, Allen AC, Gray JH, Young DC, Young LM (1987) Fetal biophysical profile and perinatal death. Obstet Gynecol 70:357-360.

Battaglia FC, Meschia G (1986) An introduction to fetal physiology. Academic Press, Orlando, Florida, 1986.

Beattie RB, Dorman JC (1989) Antenatal screening for intrauterine growth retardation with umbilical artery ultrasonography. Br Med J 298:631-635.

Berman Jr. W, Goodlin RC, Heymann MA, Rudolph AM (1975) Measurement of umbilical blood flow in fetal lambs in utero. J Appl Physiol 19:1056.

Berman Jr. W, Goodlin RC, Heymann MA, Rudolph AM (1978) Effects of pharmacologic agents on umbilical blood flow in fetal lambs in titero. Biol Neonate 33:225-235.

Bilardo CM, Nicolaides KH, Campbell S (1990) Doppler measurements of fetal and uteroplacental circulations: relationship with umbilical venous blood gases measured at cordocentesis. Am J Obstet Gynecol 1990;162:115-120.

Bissonnette JM, Farrell RC: Pressure-flow and pressure-volume relationships in the fetal placental circulation (1973). J Appl Physiol 35(3):355-360.

Blanco CE, Dawes GS, Hanson MA, McCooke HB (1984) The response to hypoxia of arterial chemoreceptors in fetal sheep and new-born lambs. J Physiol 351:25-37. 
Block BSB, LIanos AJ, Creasy RK (1984) Responses of the growth-retarded fetus to acute hypoxemia. An J Obste! Gynecol 148:878-885.

Bocking AD, Gagnon $R$, White SE, Homan J, Mine KM, Richardson BS (1988) Circulatory responses to prolonged hypoxemia in fetal sheep. Am J Obstet Gynecol 159:1418-1424.

Bracero LA, Beneck D, Kirshenbaum N, Peiffer M, Stalter P, Schulman H (1989) Doppler velocimetry and placental disease. Am J Obstet Gynecol 161:388-393.

Brosens I, Dixon $H C_{\text {, }}$ Robertson WB (1977) Fetal growth retardation and the arteries of the placental bed. Br J Obstet Gynaecol 84:656-663.

Burns PN (1988a) Hemodynamics. In: Taylor KJW, Burns PN, Wells PNT (eds): Clinical applications of Doppler ultrasound. Raven Press, New York, 1988, pp. 46-77.

Burns PN (1988b) Doppler ultrasound in obstetrics. In: Taylor KJW, Burns PN, Wells PNT (eds): Clinical applications of Doppler ultrasound. Raven Press, New York, 1988, pp. 263-303.

Buys Ballot CHD (1845) Akustische Versuche auf der Niederländische Eisenbahn, nebst gelegentlichen Bemerkungen zur Theorie des Hrn. Prof. Doppler. Poggendorf Annalen Bd $66: 321-351$.

Caldeiro-Barcia R, Casacuberta C, Burtos R, Giussi G, Gulïn L, Escarcena BS, MendesBauer C (1968) Correlation of intra-partum changes in fetal heart rate with fetal blood oxygen and acid-base state. In: Adamsons K (ed): Diagnosis and treatment of the fetal disorders. Springer Verlag, Berlin, 1968.

Callagan DA, Rowland TC, Goldman DE (1964) Ultrasonic Doppler observation of the fetal heart. Obstet Gynecol 23:637.

Cameron AD, Nicholson SF, Nimrod CA, Harder JR, Davies DM (1988) Doppler waveforms in the fetal aorta and umbilical artery in patients with hypertension in pregnancy. Am J Obstet Gynecol 158:339-345.

Carter AM (1975) Placental circulation. In: Steven DH (ed): Comparative placentation: essays in structure and function. Academic Press, London, 1975.

Charlton V, Johengen M (1987) Fetal intravenous nutritional supplementation ameliorates the development of embolization-induced growth retardation in sheep. Pediatr Res 22:55-61.

Cheung CY (1989) Direct and neural components of the fetal adrenal medullary catecholamine response to hypoxia. Society for Gynecologic Investigation. San Diego, California, 1989, abstract 68.

Clapp JF, Szeto HH, Larrow R, Hewitt J, Mann LI (1980) Umbilicall blood flow response to embolization of the uterine circulation. Am J Obstet Gynecol 138:60-67.

Clapp JF, Mann LI, Peress NS, Sxeto HH (1981) Neuropathology in the chronic fetal lamb preparation: Structure-function correlates under different environmental conditions. Am J Obstet Gynecol 141:973-986.

Cohn HE, Sacks EJ, Heymann MA, Rudolph AM (1974) Cardiovascular responses to hypoxemia and acidemia in fetal lambs. Am J Obstet Gynecol 120:817-824.

Cohn HE, Jackcon BT, Piasecki GJ, Cohen WR, Novy MJ (1985) Fetal cardiovascular responses to asphyxia induced by decreased uterine perfusion. J Dev Physiol 7:289-297.

Cohnstein $J \&$ Zuntz $\mathbf{N}$ (1884) Untersuchungen ueber das Blut, den Kreislauf und die Athmung beim Säugetier Foetus. Pllueglers Arch Ges Physiol 34:173

Cohnstein J \& Zuntz N (1888) Weitere Untersuchungen zur Physiologie des Säugetier-Foetus. Pflueglers Arch Ges Physiol 42:342. 
Colton T (1974) Statistics in mediche. 1stedition pp. 200-202. Little, Brown and Company, Boston, 1974 .

Crawford JM (1962) Vascular anatomy of the human placenta. Am J Obstet Oynecol 84:1543-1567.

Creasy RK, Barrett CT, de Swiet M, Kahanpää KV, Rudolph AM (1972) Experimental intrauterine growth retardation in the sheep. Am J Obstet Gynecol 112:566-573.

Creasy RK, Barrett CT, de Swiet M, Kahanpää K, Rudolph AM (1973) Pathophysiological changes in the foetal lamb with growth retardation. In: Foetal and Neonatal Physiology; pp. 398-403. Cambridge University Press, Cambridge, 1974.

Daffos F, Cappella-Kaplovsky M, Fonestier F (1983) Fetal blood sampling via the umbilical cord using a needle guided by ultrasound. Report of 66 cases. Prenat Diagn 3:271-2777.

Dalton KJ, Dawes GS, Patrick JE (1977) Diurnal, respiratory and other rythms of fetal lambs. Am J Obstet Gynecol 127:414-424.

Dawes GS (1962) The umbilical circulation. Am J Obstet Gynecol 84:1634-1648.

Dawes GS (1968) Foetal and neonatal physiology. Yearbook Medical Publishers Inc., Chicago. 1968.

Dawes GS, Lewis BV, Milligan JE, Roach MR, Talner NS (1968) Vasomotor responses in the hind-limbs of foetal and new-born lambs to asphyxia and aortic chemoreceptor stimulation. J Physiol 195:55-81.

Devoe LD, Castillo RA, Searle N, Searle JS (1988) Prognostic components of computerized fetal biophysical testing. Am J Obstet Gynecol 158:1144-1148.

Doppler JC (1843) Ueber das farbige licht der Doppelsterne und einiger anderer Gestime des Himmels. Abhandl d Konigl Boehmischen Gesellschaft der Wissenschaften 2:466-482.

Eik-Nes SH, Brubakk AO, Ulstein MK (1980) Measurement of human fetal blood flow. Br Med J ii:243-284.

Eik-Nes SH, Marsal K, Brubakk AO, Kristofferson K, Ulstein K (1982) Ultrasonic measurement of human fetal blood flow. J Biomed Engng 4:28-36.

Eik-Nes SH, Marsal K, Kristofferson K (1984) Methodology and basic problems related to blood flow studies in the human fetus. Ultrasound Med Biol 10:329-337.

England J (1975) Medical research. A statistical and epidemiological approach. Churchill Livingstone, Edinburgh, 1975 , p. 5.

Erskine RLA, Ritchie JWK (1985) Umbilical artery blood flow characteristics in normal and growth-retarded fetuses. Br J Obstet Gynaecol 92:605-610.

Feinkind L, Abulafia O, Delke I, Feldman J, Minkoff H (1989) Screening with Doppler velocimetry in labor. Am J Obster Gynecol 161:765-770.

Ferrazzi E, Pardi G, Bauscaglio M, Marconi AM, Gementi B, Bellotti M, Makowski EL, Battaglia FC (1988) The correlation of biochemical monitoring versus umbilical flow velocity measurements of the human fetus. Am J Obstet Gynecol 159:1081-1087.

FitzGerald DE \& Drumm JE (1977) Non-invasive measurement of human fetal circulation using ultrasound: a new method. $\mathrm{Br}$ Med J ii:1450-1451.

Fleischer A, Schulman H, Farmakides G, Bracero L, Btattner P, Randolph G (1985) Umbilical arytery velocity waveforms and intrauterine growth retardation. Am J Obstet Gynecol 151:502-505.

Fleiss J (1986) The design and analysis of clinical experiments. New York, Wiley, 1986, pp. 1-31. 
Fok RY, Pawlova Z, Benirschke K, Paul RH, Platt LD (1990) The correlation of arterial lesions with umbilical antery Doppler velocimetry in the placentas of small-for-dates pregnancies. Obstet Gyrecol 75:578-583.

Fox H (1986) Pathology of the placenta. Clin Obstet Gynaecol 13(3):501-519.

Giles WB, Trudinger BJ, Baird PJ (1985) Fetal umbilical antery flow velocity waweforms and placental resistance; pathological correlation. Br J Obstet Gynaecol 92:31-38.

Gill RW (1979) Pulsed Doppler with B-mode imaging for quantitative blood flow measurement. Ultranound Med Biol 5:223-235.

Gill RW (1985) Measurement of blood flow by ultrasound: accuracy and sources of error. Ultrasound Med Biol 11:625-641.

Goodlin RC (1979) History of fetal monitoring. Am J Obstet Gynecol 133:323-352.

Gosling RG \& King DH (1975) In: Marcus AW \& Adamson L (eds): Ultrasonic angiology in arteries and veins. Churchill Livingstone, Edinburgh, 1975.

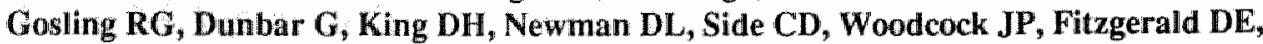
Keates JS, MacMillan D (1971) The quantitative analysis of occlusive peripheral arterial disease by a non-intrusive ultrasonic technique. Angiology 22:52-55.

Grant A, Elbourne D, Valentin L, Alexander S (1989a) Routine formal fetal movement counting and risk of antepartum fetal death in normally formed singletons. Lancet ii, 345-349.

Grant A, O'Brien N, Joy M-T, Hennessy E, MacDonald D (1989b) Cerebral palsy among children born during the Dublin randomised trial of intrapartum monitoring. Lancet, ii: 1.233-1235.

Griffin D, Bilardo K, Masini L, Diaz-Recasens J, Pearce JM, Wilson K, Campbell S (1984) Doppler flow velocity waveforms in the descending aorta of the human fetus. Br J Obstel Gynaecol 91:997-1006.

Gu W, Jones CT, Parer JT (1985) Metabolic and cardiovascular effects on fetal sheep of sustained reduction of uterine blood flow. J Physiol 368:109-129.

Gudmundsson S, Fairlie F, Lingman G, Marsal K (1990) Recording of blood velocity waveforms in the uteroplacental and umbilical circulation: reproducibility study and comparison of pulsed and continuous wave Doppler ultrasonography. J Clin Ultrasound 18:97101.

Hackett GA, Campbell S, Gamsu H, Cohen-overbeek T, Pearce JMF (1987) Doppler studies in the growth retarded fetus and prediction of neonatal necrotising enterocolitis, hatemorthage, and neonatal morbidity. Br Med J 294:13-16.

Hanretty KP, Primrose MH, Neilson JP, Whittle MJ (1989) Pregnancy screening by Doppler uteroplacental and and umbilical artery waveforms. Br J Obstet Gynaecol 96:1163-1167.

Hanson MA (1988) The importance of baro- and chemoreflexes in the control of the fetal cardiovascular system. I Dev Physjol 10:491-511.

Marding JE, Jones CT, Robinson JS (1985) Studies on experimental growth retardation in fetal sheep. The effects of a small placenta in restricting transport to and growth of the fetus. J Dev Physiol 7:427-442.

Harper MA, Murnaghan GA (1989) Discordant umbilical artery flow velocity waveforms and pregnancy outcome. Br J Obstet Gynaecol 96:1449-1452.

Harvey W (1628) Excercitatio anatomica de motu cordis et sanguinis in animalibus. Francofurti, sumpt. G. Fitzeri.

Haverkamp AD, Thompson AD, McFee JG, Cetrolu C (1976) The evaluation of continuous fetal heart rate monitoring in high-risk pregnancy. Am J Obstet Gynecol 125:310-317. 
Hill LM, Breckie R, Wolfgram KR, O'Brien PC (1986) Evaluation of three methods for estimating fetal weight. J Clin Ultrasound 14:1711/76.

Ho P-H, Melbin J, Noordergraaf A (1987) Hemodynamic principles relevant to maternal-fetal Doppler investigation. In: Reproductive and Perinatal Medicine (VII), Maulik D, McNellis D (eds): Doppler ultrasound measurement of matemal-fetal hemodymamics, pp. 1-11. Perinatology Press 1987, Ithaca, New York, 1987.

Hoddick WK, Callen PW, Filly RA, Creasy RK (1984) Ultrasonographic determination of quantitative amniotic fluid volume in intrauterine growth retardation: reassessment of the $\mathbb{1}$ $\mathrm{cm}$ rule. Am J Obstet Gynecol 149:758-762.

Hoeks APG (1982) On the dewelopment of a multi-gate pulsed Doppler system. Thesis, Maastricht, The Netherlands, 1982.

Hon EH, Quilligan EJ (1968) Electronic evaluation of fetal heart rate. IX. Further observations on "pathologic" bradycardia. Clin Obstet Gynecol 11:145-167.

Hon EH, Paul RH, Hon RW (1972) Electronic evaluation of fetal heart rate. XI. Description of a spiral ellectrode. Obstet Gynecol 40:362-365.

Huisseling van H, Hasaart THM, Muijsers GJJM, de Haan J (1990) Umbilical artery pulsatility index and placental vascular resistance during hypoxemia in fetal lambs. Society for Gynecological Investigation, St. Louis, Missouri, 1990, abstract 315.

Huisseling van H, Hasaart THM, Ruissen CJ, Muijsers GJJM, Haan de J (1989) Umbilical artery flow velocity waveforms during acute hypoxemia and the relation with hemodynamic changes in the fetal lamb. Am J Obstet Gynecol 161:1061-1064.

Iwamoto HS, Rudolph AM (1981) Effects of angiotensin II on the blood flow and its distribution in fetal lambs. Circ Res 48:183-189.

Johnson R (1984) Elementary statistics. PWS Publishers, Boston, Massachusetts, 1984, 4th edition, pp. 434-468.

Jones CT, Roebuck MM, Walker DW, Johnston BM (1988) The role of the adrenal medulla and peripheral symapthetic nerves in the physiological responses of the fetal sheep to hypoxia. J Dev Physiol 10:17-36.

Jones CT, Robinson RO (1975) Plasma catecholamines in foetal and adult sheep. J Physiol 248:15-33.

Jouppila $\mathbf{P}$, Kirkinen $\mathbf{P}(1984)$ Increased vascular resistance in the descending aorta of the human fetus in hypoxia. Br J Obstet Gynaecol 91:853-856.

Jouppila P, Kirkinen P (1986) Blood velocity waveforms of the fetal aorta in normal and hypertensive pregnancies. Obstet Gynecol 67:856-860.

Khong TY, De Wolf F, Robertson WB, Brosens I (1986) Inadequate maternal vascular response to placentation in pregnancies complicated by pre-eclampsia and by small-for-gestational age infants. Br J Obstet Giynaecol 93:1049-1059.

Kidd LC, Patel NB, Smith R (1985) Non-stress antenatal cardiotocography a prospective randomized clinical trial. Br J Obstet Gynaecol 92:1156-1159.

Lejumeau de Kergaradec (1821) Memoire de l'auscultation, appliquée a l'étude de la grossesse.

Low JA, Galbraith RS, Muir DW, Killen HL, Pater EA, Karchmar EJ (1985) The relationship between perinatal hypoxia and newborn encephalopathy. Am J Obstet Gynecol $152: 256$ 
MacDonald D, Grant A, Sheridan-Pereira $\mathbf{M}_{3}$ Boylan $\mathbf{P}$, Chalmers I (1985) The Dublin randomized controlled trial of intrapartum fetal heart rate monitoring. Am J Obstet Gynecol 152.524539 .

Makowski EL (1968) Maternal and fetal wascular nets in placentas of sheep and goats. Am J Obstet Gynecol 100:283-288.

Makowski EL, Schneider JM, Tsoulos NG, Colwill JR, Battaglia FC, Meschia G (1968) Cerebral blood flow, oxygen consumption and glucose utilization of fetal lambs in utero. Am J Obstet Gynecol 114292-303:

Mamning A A, Platt LD, Sipos L (1980) Antepartum fetal evaluation: Development of a fetal biophysical profile. Am J Obstet Gynecol 136:787-795.

Manning FA, Lange IR, Morrison I, Harman CR (1984) Fetal biophysical profile score and the nonstress test: a comparative trial. Obstet Gynecol 64:326-331.

Martin CB, de Haan $\mathfrak{J}$, van der Wildt $\mathbf{B}$, Jongsma $\mathbf{H W}$, Dieleman A, Arts THM (1979) Mechanisms of Jate decelerations in the fetal heart rate. A study with autonomic blocking agents in fetal lambs. Eur J Obstet Gynecol reprod Biol 9:361-373.

Martin AA, Kapoor R, Scroop GC (1987) Hormonal factors in the control of heart rate in normoxaemic and hypoxaemic fetal, neonatal and adult sheep. J Dev Physiol 9:465-480.

Maulik D, Yarlagadda AP, Youngblood JP, Willoughby L (1989) Components of variability of umbilical arterial Doppler velocimetry a prospective analysis. Am J Obstet Gynecol 160:1406-1412.

McCowan LM, Mullen BM, Ritchie JWK (1987) Umbilical artery flow velocity waveforms and the placental vascular bed. Am J Obstet Gynecol 157:900-902.

McDonald DA (1974) Blood flow in arteries. Edward Arnold, London, 1974, 2nd edition, p. 333.

MeGalluim WD, Willams CS, Napel S, Daigle RE (1978) Fetal blood velocity waveforms. Am J Obstet Gynecol 132:425-429.

Meschia G, Cotter JR, Makowski EL, Barron DH (1967) Simultaneous measurement of uterine and umbillical blood flows and oxygen uptakes. Q J Exp Physiol 52:1-18.

Milnor WR (1972) Pulsatile blood flow. N Engl J Med 287:27-34.

Mohide P, Keirse MJNC (1989) Biophysical assessment of fetal well-being. In: Chalmers I, Enkin $M$, Keirse MJNC (eds): Effective care in pregnancy and childbirtl. Oxford University Press, Oxford $_{\text {pp } 477-492 .}$.

Morrow RJ, Adamson SL, Bull SB, Ritchie JWK (1989) Effect of placental embolization on the umbilical arterial velocity waveform in fetal sheep. Am J Obster Gynecol 161:10551060.

Morrow RJ, Adamson SL, Bull SB, Ritchie JWK (1990) Acute hypoxemia does not affect the umbilical artery flow velocity waveform in fetal sheep. Obstet Gynecol 75:590-593.

Mott JC (1982) Control of the foetal circulation. J Exp Biol 100:129-146.

Muijsers GJJM, Hasaart THM, Ruissen CJ, van Huisseling H, Peeters LLH, de Haan J (1990a) The response of the umbilical and femoral artery pulsatility indices in fetal sheep to progressively reduced uterine blood flow. J Dev Physiol, in press.

Muijsers GJJM, van Huisseling H, Hasaart THM (1990b) The effect of selective umbilical embolization on the common umbilical artery pulsatility index and umbilical vascular resistance in fetal sheep. J Dev Physiol accepted for publication 1990. 
Mulders LGM, Muijsers GJIM, Jongsma HW, Nijhuis JG, Hein PR (1986) The umbilical artery blood flow velocity waveform in relation to fetal breathing movements, fetal heart rate and fetal behavioural states in normal pregnancy at $37-39$ weeks. Early Hum Dewelop 14:283-293.

Mulders LGM, Wijn PFF, Jongsma HW, Hein PR (1987) A comparative stody of three indices of umbilical blood flow in relation to prediction of growth retardation. J Perinat Med 15:3-12.

Myers RE, Beard R, Adamson K (1969) Brain swelling in the newborn rhesus monkey folllowing prolonged partial asphyxia. Neurology 19:1012-1018.

Myers RE (1972) Two patterns of perinatal brain damage and their conditions of occurrence. Am J Obster Gynecol 112:246-176.

Nathanielsz PW, Maulik D, Yarlagadda P, Figueroa J (1987) Animal models for the study of matemal-fetal physiology and pathophysiology. In: Maulik D, McNellis D (eds): Doppler ultrasound measurement of maternal-fetal hemodynamics. Perinatology Press, Ithaca, New York, 1987 , pp. 283-299.

Neilson JP (1987) Commentary: Doppler ultrasound. Br J Obstet Gynaecol 94:929-932.

Nelson KB, Ellenberg JH (1986) Antecedents of cerebral palsy: multivariate analysis of risk. N Engl J Med 315:81-86.

Nelson KB, Ellenberg JH (1981) Neonatal signs as predictors of cerebral palsy. Pediatrics 64:225-232.

Newnham JP, Patterson LL, James IR, Diepeveen DA, Reid SE (1990) An evaluation of the efficacy of Doppler flow welocity waveform analysis as a screening test in pregnancy. Am J Obstet Gynaecol 162:403-410.

Newnham JP, Kelly RW, Roberts RV, Macintyre M, Speijers J, Johnson T, Reid SE (1987) Fetal and maternal flow welocity waveforms in normal sheep pregnancy. Placenta 8:125-136.

Nicolaides KH, Soothill PW, Rodeck CH, Campbell S (1986) Ultrasound guided sampling of umbilical cord and placental blood to assess fetal wellbeing. Lancet ii, 1065-1067.

Nienhuis SJ, wan Vugt, Hoogland $H J$, Ruissen CJ, de Haan J (1988) Interexaminer variability study of fetal velocity waveforms. Gynecol obster Invest 25:152-157.

Noordam MJ, Wladimiroff JW, Lotgering FK, Struijk PC, Tonge HM (1987) Fetal blood flow velocity waveforms in relation to changing peripheral vascular resistance. Early Hum Develop 15:119-127.

O'Rourke MF, Taylor MG (1966) Vascular impedance in the fermoral bed. Circ Res 18:126

O'Rourke MF (1982) Vascular impedance in studies of arterial and cardiac function. Physiol Rev 62:570-623.

Parer JT (1980) The effect of acute maternal hypoxia on fetal oxygenation and the umbilical circulation in the sheep. Eur J Obstet Gynecol reprod Biol 10:125-136.

Parer JT, Dijkstra HR, Vredebrecht PPM, Harris JL, Krueger TR, Reuss ML (1980) Increased fetal heart rate variability with acute hypoxia in chronically instrumented sheep. Eur J Obstet Gynecol reprod Biol 10:393-399.

Parer JT (1983) The influence of beta-adrenergic activity on fetal heart rate and the umbilical circulation during hypoxia in fetal sheep. Am J Obstet Gynecol 147:592-597.

Paulick RP, Meyers RL, Rudolph CD, Rudolph AM (1990) Venous responses to hypoxemia in the fetal lamb. J Dev Physiol, submitted for publication 1990. 


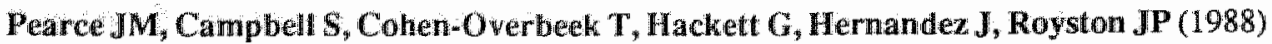
References ranges and sources of wariation for indices of pulsed Doppler flow welocity wavefoms from the ateroplacental and fetal circulation. Br J Obstet Gynaecol 95:248-256.

Peeters LLH, Sheidon RE, Jones MD, Makowski EL, Meschia G (1979) Blood flow to fetal organs as a function of arterial oxygen content. Am J Obstet Gynecol 135:637-646.

Plainiol TH, Pourcelol L (1974) Doppler effect study of the carotid circulation. In: Vlieger M, White DN, McCready VR (eds): Ultrasonics in medecine. Excerpta Medica, Amsterdam, 1974 .

Platt LD, Walla CA, Paul RH, Trujillo ME, Loesser CV, Jacobs ND, Broussard PM (1985) A prospeetive trial of the fetal biophysical profile versus the nonstress test in the management of high-risk pregnancy. Am J Obstet Gymecol 153:624-633.

Pocock SJ (1983) Clinical trials: a practical approach. John Wiley \& Sons, Chicester, 1983.

Prentice A, Lind T (1987) Fetal heart monitoring during labour: too frequent intervention, too little benefit? Lancet, ii, 1375-1377.

Ramsey EM, Donner MW (1980) Placental vasculature and circulation. Georg Thieme Publishers, Stuttgart, 1980.

Redman CWG (1989) Examination of the placental circulation by Doppler ultrasound. Its place ir management still to be defined. Br Med J 298:62 1 622 .

Reuwer PJHM, Nuyen WC, Beijer HJM, Heethaar RM, Bruinse HW, Stoutenbeek P, Haspels AA (1984a) Characteristics of flow velocities in the umbilical arteries, assessed by Doppler ultrasound. Eur J Obstet Gynecol reprod Bioll 1:7:394-408.

Reuwer PJHM, Bruinse HW, Stoutenbeek P, Haspels AA (1984b) Doppler assesment of the fetoplacental circulation in normal and growth-retarded fetuses. Eur J Obstet Gynecol reprod Biol 18:199-205.

Reuwer PJHM, Nuyen WC, Beijer HJM, Heethaar RM, Haspels AA, Bruinse HW (1986a) Feto-placental circulatory competence. Eur J Obstet Gynecol reprod Biol 21:15-26.

Reuwer PJHM (1986b) Doppler assessment of feto-placental circulatory competence. Thesis, Utrecht, The Netherlands, 1986.

Reuwer PJHM, Sijmons CA, Rietman GW, van Tiel MWM, Bruinse HW (1987) Intrauterine growth retardation: prediction of perinatal distress by Doppler ultrasound. Lancet $11: 415 \times 418$.

Rochelson B, Schulman H, Fleischer A, Farmakides G, Bracero L, Ducey L, Winter D, Penny B (1987b) The clinical significance of Doppler umbilical artery velocimetry in the small for gestational fetus. Am J Obstet Gynecol 156:1223-1226.

Rochelson B, Schulman H, Farmakides G, Bracero L, Ducey L, Fleischer A, Penny B, Winter D (1987a) The significance of absent end-diastolic velocity in umbilical artery velocity waveforms. Am J Obstet Gynecol 156:1213-1218.

Rodeck CH, Campbell S (1978) Sampling pure fetal blood by fetoscopy in the second trimester of pregnancy. Br Med J in:728-730.

Rudolph AM, Heymann AM (1967) The circulation of the fetus in utero. Circ Res 21:163-184.

Rudolph AM, Heymanm MA (1970) Circulatory changes during growth in the fetal lamb. Circ Res 26:289-299.

Rudolph AM (1976) Factors affecting umbilical blood flow in the lamb in utero. Proceedings of the 5th European Congress of Perinatal Medicine, Uppsala, Sweden, 1976.

Rudolph AM (1984) The fetal circulation and its response to stress. J Dev Physiol 6:11-19. 
Ruilssen CJ, van Vugt JMG, Hoogland HJ, Hoeks APG, de Han J (1987a) Technical aspects of fetal Doppler measurements. Gynecol obstet lnvest $24: 1-13$.

Ruissen CJ, van Vugt JMG, de Haan J (1987b) Variability of PI calculations, Eur J Obstet Gynecol reprod Biol 27:213-220.

Satomura $\mathbf{S}(1959)$ Study of flow pattem in peripheral anteries by ultrasonics. I Acoust Soc Japan 15:151-159.

Scheffen I, Phillipens L, Kaufman P, Leiser $\mathbf{R}_{,}$Mironov V (1989) Matemal oxygen supply as a regulator of fetal placental capillarisation. Placenta 10:501.

Schulman H, Fleischer A, Stern, W, Farmakides G, Jagani N, Blattner P (1984) Umbilical velocity wave ratios in human pregnancy. Am IObstet Gynecol 148:985-990.

Shaw KJ, Medearis AL, Walla C, Platt LD (1990) Logistic regression analysis of the biophysical profile in the prediction of fetal distress. Society for Gynecologic Investigation, St. Louis, Missouri, 1990 , abstract 400.

Sijmons EA, Reuwer PJHM, van Beek E, Bruinse (1989) The validity of screening for small-for-gestational-age and low-weight-for-length infants by Doppler ultrasound. Br I Obstet Gynaecol 96:557-561.

Skidmore R, Woodcock JP, Wells PNT, Bird D, Baird RN (1980) Physiological interpretation of Doppler-shift waveforms III. Ultrasound Med Biol 6:227-231.

Skillman CA, Plessinger MA, Woods JR, Clark KE (1985) Effect of graded reductions in uteroplacental blood flow on the fetal lamb. Am J Physiol 249:1098-1105.

Stuart B, Drumm B, FitzGerald DE, Duignan NM (1980) Fetal blood velocity waveforms in normal pregnancy. Br J Obster Gynaecol 87:780-785.

Taylor KJW, Burns PN, Wells PNT (1988) Clinical applications of Doppler ultrasound. Raven Press, New York, 1988.

Thacker $\mathbf{S}$ (1987) The efficacy of intrapartum fetal monitoring. Am $\mathrm{J}$ Obstet Gynecol 156:24-30.

Thacker SB, Berkelman RL (1986) Assessing the diagnostic accuracy and efficacy of selected antepartum fetal surveillance techniques. Obstet Gynecol Survey $41: 121-141$.

Thompson RS, Trudinger BJ, Cook CM (1986) A comparison of Doppler ultrasound waveform indices in the umbilical artery 1 . indices derived from the maximum velocity waveform. Ultrisound Med Biol 12:835.844.

Tonge HM, Wladimiroff JW, Noordam MJ, van Kooten C (1986) Blood flow velocity waveforns in the descending aorta: comparison between normal and growth-retarded pregnancies. Obstet Gynecol 67:851-855.

Trudinger BJ, Giles WB, Cook CM, Bombardieri J, Collins L (1985) Fetal umbilical artery flow velocity waveforms and placental resistance: clinical significance. $\mathrm{Br} J$ Obstet Gynaecol 92:23-30.

Trudinger BJ, Cook CM, Jones L, Giles WB (1986) A comparison of fetal heart rate monitoring and umbilical artery waveforms in the recognition of fetal compromise. Br J Obstet Gynaecol 93:171-175.

Trudinger BJ, Cook CM, Giles WB, Connelly A, Thompson RS (1987a) Umbilical artery flow velocity waveforms in high-risk pregnancy. Randomised controlled trial. Lancet ii: $188-190$. 
Trudinger BJ, Stevens D, Condly A, Halles JRS, Alexander G, Bradley L, Fawcett A, Thompson $\mathbf{R}$ (1987b) Umbilical ariery flow velocity waveforms and placental resistance: the effects of embolization of the umbilical circulation. Am J Obstet Gynecol 157:14431448.

Trudinger BJ, Cook CM (1988) Different umbilical artery flow velocity waveforms in one patient. Obstet Gynecol 71:1019-1021.

Trudinger BJ, Cook CM (1990) Doppler umbilical and uterine flow waveforms in severe pregnancy hypertension. Br J Obstet Gynaecol 97:142-148.

Vintzileos AM, Campbell WA, Ingardia CJ, Nochimson DJ (1983) The fetal biophysical profle and its predictive walue. Obstet Gynecol 62:271-278.

Vintwileos AM, Gaffney SE, Salinger LM, Kontopoulos VG, Campbell WA, Nochimson, DJ (1987) The relationships among the fetal biophysical profile, umbilical cord $\mathrm{pH}$ and Apgar scores. Am J Obstet Gynecol 157:627-631.

Volpe $J \mathbf{J}$ (1977) Observing the infant in the early hours after asphyxia. In: Gluck L (ed): Intraterine asphyxia and the deweloping brain, pp. 263-283. Chicago, Year Book Publishing Company, 1977.

Vugt van JMG, Ruissen CJ, Hoogland H.J, de Haan J (1987a) A prospective study of the umbilical artery blood flow velocity waveform in appropriate-for-date and growth-retarded fetuses. Gynecol obstet Invest 23:217-225.

Vugt van JMG, Ruissen CJ, Hoogland HJ, de Haan J (1987b) A prospective study of velocity waveforms in the fetal descending and and abdominal aorta in fetuses appropriatefor-gestational-age and in growth-retarded fetuses. Gynecol obstet Invest 24:14-22.

Vugt van JMG, Hasaart THM, Ruissen CJ, Hoogland HJ, Hoeks APG, de Haan J (1988a) The pulsatility index and its relationship to placental vascular resistance during partial umbilical venous occlusion: a study in fetal lambs. Gynecol obstet Invest 26:1-7.

Vugt wan JMG, Ruissen KJ, Schouten HJA, Theunissen M, Hoogland HJ, de Haan J (1988b). Umbilical artery blood velocimetry: a prospective longitudinal study in search of the intrauterine growth-retarded fetus. Early Hum Develop 18:59-71.

Vugt van JMG (1988c) Fetal artery Doppler velocimetry. Thesis, Maastricht, The Netherlands, 1988.

Vyas S, Nicollaides $\mathbf{K H}$, Campbell S (1989) Renal artery flow-velocity waveforms in normal and hypoxemic fetuses. Am J Obster Gynecol 161:168-172.

Walker $\mathbf{N}(1959)$ The case for conservatism in management of fetal distress. Br Med J ii: $1221-1226$.

Wallenstein S, Zucker CL, Fleiss JL (1979) Some statistical methods useful in circulation research. Circ Res 47:1-9.

Weiner CP (1990) The relationship between the umbilical artery systolic/diastolic ratio and umbilical blood gas measurements in specimens obtained by cordocentesis. Am J Obstet Gynecoll 162:1198-1202.

Wheble AM, Gillmer MD, Spencer JA, Sykes GS (1989) Changes in fetal monitoring practice in the UK: 1977-1984. Br J Obstet Gynaecol 1140-1147.

Wimsatt WA (1962) Some aspects of the comparative angtomy of the mammalian placenta. Am J Obstet Gynecol 84:1568-1594.

Winckell F (1893) Lehrbuch der Geburtshilfe. Leipzig, 1893.

Wladimiroff JW, Tonge HM, Stewart PA (1986) Doppler ultrasound assessment of cerebral blood flow in the human fetus. Br J Obstet Gynaecol 93:471-475. 
Woo JSK, Liang ST, Lo RLS, Chan FY (1987) Middle cerebral artery Doppler flow velocity waveforms. Obstet Gynecol 70:613-617.

Yarf H, Parer JT, Block BS, Llanos AJ (1987) Cardiorespiratory responses to graded reductions of uterine blood flow in the sheep fetus. J Dew Physiol 9:325-336.

Zweifel P (1876) Die respirstion des Foetus. Arch Gynaek 9:291-305. 


\section{Summary}

Hypoxia is regarded as an important cause of perinatal mortality and morbidity. Currently, the only method for direct determination of fetal hypoxia is the analysis of a blood sample from the umbilical cord. The blood vessels in the umbilical cord can be visualized and sampled using two-dimensional ultrasound. This procedure however, is hampered by a number of disadvantages. In obstetric practice, diagnostic tests should not be dangerous or discomfortable for mother and fetus. Furthermore, they should be easily and repetitively applicable in all pregnant women at any time. Cardiotocography, which includes simultaneous registration of fetal heart rate and uterine activity, is an example of such a method. The fetal condition is subsequently assessed on the basis of criteria. However, whether application of cardiotocography indeed results in an improved maternal and fetal outcome is questionable. If such an improved outcome exists, the benefit is probably limited to high-risk pregancies.

Amongst both clinicians and investigators, there is much interest in a method which application in pregnant women was firstly described in 1977. This method is characterized by registration and analysis of blood flow velocity waveforms in fetal vessels. Blood flow velocity waveforms can be obtained by emission of ultrasound in the direction of a blood flow. The ultrasound is then dispersed and reflected, while a frequency shift occurs, which depends on the direction and velocity of the blood flow. This frequency shift is known as the Doppler effect. After electronic processing, the received signal can be made audible and visible. The blood flow velocity waveform is often defined as the envelope curve around the visible signal. From such a waveform, which occurs in arteries after each cardiac contraction, parameters can be calculated which provide essential information on the blood flow characteristics in the vessel. In the studies described in this thesis, waveform analysis was mainly established using the Pulsatility Index. The Pulsatility Index provides, from the parameters used in obstetrics, the most accurate description of the blood flow velocity waveform. The Pulsatility Index is associated with the impedance to blood flow at the point of measurement. On the basis of this association., it might be possible to get insight in the blood flow distribution within the fetus. This hypothesis was used when the study was designed. Fetal hypoxia results in a 
redistribution of blood flow. The blood flow to myocardium, brain and adrenals increases, whereas the blood flow to lungs, kidneys, intestines, bones, muscles and skin decreases. Fetal blood flow to the placenta remains constant or increases slightly. The latter phenomenon suggests that during fetal hypoxia, umbilical vascular resistance does not significantly change. However, it has been demonstrated that the umbillical artery Pulsatility Index might be increased in infants who suffer from perinatal asphyxia or who are small for gestational age. Therefore, it has been hypothesized that the umbilical artery Pulsatility Index can provide information on fetal wellbeing. This hypothesis however, is contradictory with the former assumption that umbilical vascular resistance does not significantly change during fetal hypoxia. This controversion raises questions concerning the relationship between (the Pulsatility Index of) fetal blood flow velocity waveforms on the one hand, and fetal oxygen availability and vascular resistance on the other hand. In this thesis, the relationship between fetal oxygen availability and fetal blood flow velocity waveforms was studied in various ways. The studies were performed in the pregnant sheep, an animal model of human pregnancy. To this end, the ewes and fetal lambs were surgically provided with measuring instruments.

In chapter 3 , an experiment is described in which uteroplacental blood flow is gradually but progressively reduced using a vessel occluder around the maternal common internal iliac artery. This resulted in a progressive fetal hypoxemia, which was associated with an increase in fetal mean arterial pressure and with an increase in the Pulsatility Index in the femoral arteries, which predominantly supply the bones, muscles and skin of the lower legs. Fetal heart rate remained relatively stable, probably because of the very gradual decrease in oxygenation. Also the umbilical artery Pulsatility Index did not change, which indicates that fetal hypoxemia can coexist with a normal umbilical artery blood flow velocity waveform. Subsequently, it was examined whether a relationship could be found between the ratio of simultaneous Doppler measurements in fetal vessels on the one hand, and fetal oxygen content on the other hand (chapter 4). In order to prevent fetal acidemia on the basis of hypercarbia, the ewe was provided with a low oxygen gas mixture. The results strongly agreed with those of the former experiment: no significant changes in fetal heart rate and in umbilical artery Pulsatility Index. However, in some fetal lambs, the expected increase in femoral artery Pulsatility Index did not occur, while the expected increase in fetal mean arterial pressure was also absent. A weak correlation was found between the ratio of Doppler measurements in the femoral and umbilical artery on the one hand, and fetal oxygen content on the other hand. It is therefore suggested that the diagnostic value of Doppler measurements for the assessment of fetal hypoxemia might be low. However, a 
strong correlation was found between the femoral artery Pulsatility Index and mean arterial pressure in each of the fetal lambs examined. This correlation is explained by the fact that both phenomena probably result from the same mechanism, i.e. arteriolar vasoconstriction in the lower legs.

Since acute hypoxemia during several hours did not result in changes in the umbilical artery blood flow velocity waveform, it was decided to examine the effect of prolonged hypoxemia during several days (chapter 5). A model was used which may resemble to the "placental insufficiency syndrome", known from human obstetrics. This model was characterized by embolization of the matemal uteroplacental vessels using microspheres. The embolization procedure was repeated daily, until fetal oxygenation had decreased below a certain level. The average survival period after the first embolization procedure was 3 days. This period was characterized by a progressive hypoxemia and acidemia. Also fetal mean arterial pressure increased gradually. No significant changes were observed in both fetal heart rate and umbilical artery Pulsatility Index. It was concluded that fetal hypoxemia can exist without any functional or structural changes in fetal placental blood vessels that result in an increase in umbilical artery Pulsatility Index. It was thus demonstrated once more that fetal hypoxemia can coexist with normal umbilical artery blood flow velocity waveforms. In the meantime, it had appeared from mathematical models and from anatomical and experimental studies that abnormal fetal placental blood flow velocity waveforms are probably explained by a reduced placental perfusibility. According to most authors, such a reduced perfusibility probably results from increased vascular resistance. Using selective embolization of the fetal placental circulation, it was possible to examine the relationship between umbilical vascular resistance and the umbilical artery Pulsatility Index. In each of the fetal lambs examined, a strong positive correlation was found between both variables. This finding confirms the hypothesis that an increased umbilical artery Pulsatility Index results from a reduced perfusibility of the fetal placental circulation.

It was already suggested by other authors that an abnormal waveform in one of both umbillical arteries does not necessarily implies fetal compromise. By comparison of waveforms obtained in an embolized region, with waveforms obtained in vessels that supply the whole placenta, it was possible to show that an abnormal waveform in one of both umbilical arteries does not necessarily imply a significantly reduced placental blood flow. The Pulsatility Index in the fetal aorta or common umbilical artery varied consistently with total umbilical blood flow, whereas the Pulsatility Index in a cotyledonary artery varied incon- 
sistently with total umbilical blood flow. It is suggested that clinical decisions should not be made on the basis of an abnormal waveform in one of both umbilical arteries.

In the assessment of fetal. health or the effect of drugs on the fetus, a change in fetal heart rate is often used as the only criterium. From experimental studies however, it appears that stress conditions are often associated with changes in fetal blood pressure. Therefore it might be essential to get insight in fetal blood pressure by means of noninvasive tools. Stress conditions are often characterized by an increase in systemic vascular resistance, which is parallelled by an increase in mean arterial pressure. We examined the relationship between mean arterial pressure and the Pulsatility Index in the fetal aorta using vasoconstrictive drugs. Only a weak positive correlation was found between both variables, probably on the basis of absence of circulatory steady state. To get insight in the relationship between mean arterial pressure and aorta Pulsatility Index, both variables should be examined in chronically instrumented fetuses at stable conditions of stress.

It is concluded that animal experiments can improve the insight into the diagnostic significance of fetal Doppler measurements. The value of Doppler measurements in obstetric decision-making should further be examined in clinical studies. 


\section{Samenvatting}

Zuurstoftekort wordt beschouwd als een belangrijke oorzaak van sterfte en ziekte bij ongeboren en pasgeboren kinderen. Het direct vaststellen van zuurstoftekort bij ongeborenen is op dit moment alleen mogelijk door onderzoek van een bloedmonster uit de navelstreng. Een bloedvat in de navelstreng kan met behulp van ultrageluid (echoscopie) in beeld gebracht worden en vervolgens worden aangeprikt. Aan deze procedure zijn echter een aantal nadelen verbonden. In de verloskundige praktijk wordt bij voorkeur gebruikt gemaakt van onderzoeksmethoden die niet gevaarlijk of belastend zijn voor moeder en ongeboren kind, bij alle zwangeren op elk gewenst moment kunnen worden toegepast, en bovendien gemakkelijk en bij herhaling toepasbaar zijn. Een voorbeeld van een dergelijke methode is cardiotocografie. Hierbij wordt een registratie gemaakt van de foetale hartfrequentie en van de baarmoeder-activiteit. Aan de hand van criteria kan vervolgens een uitspraak gedaan worden omtrent de toestand van het kind. Echter, of toepassing van cardiotocografie daadwerkelijk leidt tot verbetering van het resultaat voor zowel moeder als kind is onzeker. Indien er sprake is van een gunstig effect, dan is dit zeer waarschijnlijk alleen het geval voor zwangerschappen met een verhoogd risico.

Bij klinici en onderzoekers bestaat momenteel veel belangstelling voor een methode waarvan de toepassing bij zwangeren voor het eerst werd beschreven in 1977. Deze methode omvat het registreren en analyseren van bloedstroomsnel heidspatronen in foetale (kinderlijke) slagaderen. Bloedstroomsnelheidspatronen worden verkregen door ultrageluid onder een scherpe hoek te zenden in de richting van een bloedstroom. Het ultrageluid wordt hierbij verstrooid en weerkaatst, terwijl er een frequentie-verandering optreedt die samenhangt met de richting en de snelheid van de bloedstroom. Deze frequentie-verandering staat bekend als het Doppler-effect. Het terug-ontvangen signaal kan na electronische bewerking hoorbaar en zichtbaar gemaakt worden. De golfvorm die het zichtbare signaal omhult wordt veelal gedefiniëerd als de Doppler bloedstroomsnelheidscurve. Uit deze golfvorm, die optreedt in slagaderen in aansluiting aan de lediging van het hart, zijn parameters te berekenen die relevante informatie verschaffen omtrent kenmerken van de bloedstroom in het bloedvat. In het in dit proefschrift beschreven onderzoek werd voomamelijk gebruik gemaakt van de Pulsatility Index. Deze geeft, van de in de verloskunde toegepaste parameters, de meest 
nauwkeurige beschrijving van de bloedstroomsnelheidscurve. De Pulsatility Index wordt geassocieerd met de weerstand die de bloedstroom in het bloedvat ter plaatse van het meetpunt ondervindt. Op basis van deze associatie zou men, indien men de Pulsatility Index zou bepalen in verschillende foetale slagaderen, een indruk kunnen verkrijgen omtrent de verdeling van de bloedstroom binnen de foetus. Van deze veronderstelling is gebruik gemaakt bij de opzet van het onderzoek. Zuurstoftekort bij de foetus leidt tot een herverdeling van de bloedstroom. De bloedstroom naar hart, hersenen en bijnieren neemt toe, terwijl de bloedstroom naar de longen, nieren, darmen, beenderen, spieren en huid afneemt. De bloedstroom naar de placenta (moederkoek) blijft relatief constant of neemt iets toe. Op basis hiervan lijkt het aannemelijk dat bij zuurstoftekort de vaatweerstand in de navelstreng en placenta niet wezenlijk verandert. Anderzijds is uit onderzoek gebleken dat de Pulsatility Index in de navelstreng vaak verhoogd is bij kinderen bij wie zuurstoftekort optreedt rondom de geboorte en bij wie het geboortegewicht extreem laag is. Dit heeft geleid tot de veronderstelling dat de Pulsatility Index in de navelstrengslagader informatie zou verschaffen omtrent de gezondheidstoestand van de foetus. Deze veronderstelling is echter in tegenspraak met de eerder genoemde aanname dat de vaatweerstand in de navelstreng en placenta tijdens zuurstoftekort niet wezenlijk verandert. Deze controverse doet ondermeer vragen opwerpen met betrekking tot de relatie tussen (de Pulsatility Index van) foetalle bloedstroomsnelheidscurven enerzijds, en de foetale zuurstofvoorziening en vaatweerstand (in de navelstrengslagader) anderzijds. In het hier beschreven onderzoek werd op diverse wijzen gekeken naar de relatie tussen de foetale zuurstofvoorziening en foetale bloodstroomsnelheidscurven. Het onderzoek werd verricht in drachtige schapen, die als model fungeerden voor de humane zwangerschap. De schapen en ongeboren lammeren werden hiertoe operatief voorzien van meetinstrumenten.

In hoofdstuk 3 staat een experiment beschreven waarbij de bloedstroom naar de baarmoeder en placenta geleidelijk maar toenemend verminderd wordt door middel van een afsluitmechanisme rondom het toevoerende moederlijke bloedvat. Hierdoor ontwikkelt zich bij de foetus een toenemend zuurstoftekort. Dit zuurstoftekort is geassociéerd met een toename in de foetale bloeddruk en een toename in de Pulsatility Index van de foetale liesslagaderen, die met name de beenderen, spieren en huid van de achterste ledematen van bloed voorzien. De foetale hartfrequentie blijft vrij stabiel, waarschijnlijk doordat het zuurstoftekort zich zeer geleidelijk ontwikkelt. Ook de Pulsatility Index in de navelstrengslagader verandert niet, hetgeen aanduidt dat foetaal zuurstoftekort kan bestaan, terwijl de bloedstroomsnelheidscurve in de navelstrengslagader een normale vorm heeft. Vervolgens werd onderzocht of er een relatie bestaat tussen de ratio van gelijktijdige Doppler-metingen in foetale bloedvaten enerzijds en het foetale zuurstofge- 
halte anderzijds (hoofdstuk 4). Ter voorkoming vaneen verzuring van het foetale bloed door een toename van het koolzuurgehalte, kreeg het moederschap wern zuurstof-arm gasmengsel toegediend. De resultaten kwamen sterk overeen met die van het eerder genoemde experiment: geen wezenlijke veranderingen in de foetale hartfrequentie en in de Pulsatility Index van de navelstrengslagader. Echter de verwachte stijging van de Pulsatility Index in de liesslagader bleef in enkele lammeren achterwege, terwijl in deze lammeren ook de verwachte bloeddrukstijging achterwege bleef. Bij nadere analyse van de resultaten bleek dat er een zwakke relatie was tussen de ratio van Doppler-metingen in de liesslagader en navelstrengslagader enerzijds en het foetale zuurtstofgehalte anderzijds. Deze zwakke relatie suggereert dat de diagnostische waarde van Doppler-metingen voor het vaststellen van foetaal zuurstoftekort weleens zou kunnen tegenvallen. Echter, een sterk verband werd gevonden tussen de Pulsatility Index van de liesslagader en de bloeddruk in elk van de onderzochte lammeren. Dit verband wordt verklaard doordat een stijging in beide grootheden kan voortkomen uit een en hetzelfde fenomeen, namelijk een samentrekking van de kleine bloedvaatjes in de achterste ledematen.

Omdat acuut zuurstoftekort gedurende enkele uren geen aanleiding gaf tot veranderingen in het bloedstroomsnelheidspatroon van de navelstrengslagader, werd besloten het effect van een meer langdurig zuurstoftekort gedurende meerdere dagen te onderzoeken (hoofdstuk 5). Hierbij werd gebruik gemaakt van een model dat mogelijk gelijkenis vertoont met het begrip "placenta-insufficientie" uit de humane verloskunde. Dit model bestond uit een embolisatie van de moederlijke bloedvaatjes naar baarmoeder en placenta met behulp van zeer kleine deeltjes. De embolisatie-procedure werd dagelijks herhaald totdat het zuurstofgehalte tot een bepaald niveau was gedaald. De gemiddelde overlevingsduur na de eerste embolisatie-procedure was 3 dagen. In deze periode nam het zuurstoftekort toe, terwijl er ook een verzuring van het foetale bloed optrad. Verder was er een toename van de bloeddruk. In de foetale hartfrequentie deden zich geen grote schommelingen voor, terwijl ook de Pulsatility Index van de navelstrengsagader niet wezenlijk veranderde. Hieruit kan geconcludeerd worden dat er sprake kan zijn van zuurtoftekort bij de foetus, zonder dat dit door functionele of structurele veranderingen in de placentaire bloedvaatjes aanleiding heeft gegeven tot een stijging van de Pulsatility Index in de navelstrengslagader. Dit resultaat bevestigde andermaal de veronderstelling dat emstig foetaal zuurstoftekort kan samengaan met een normaal bloedstroomsnelheidspatroon in de navelstreng. Uit wiskundige modellen en uit anatomische en experimentele studies was inmiddels gebleken dat de meest voor de hand liggende verklaring voor afwijkende foetoplacentaire bloedstroomsnelheidscurven gelegen was in een verminderde doorstroombaarheid van de placenta. Deze verminderde doorstroombaarheid was 
volgens de meeste auteurs te wijten aan een werhoogde vaatweerstand. Door nu selectief en gelokaliseerd de bloedvaatjes van de placenta te emboliseren, was het mogelijk de relatie te onderzoeken tussen de vaatweerstand in de foetoplacentaire blooedsomloop en de Pulsatility Index in de navelstrengslagader. Er bleek sprake van een sterk positief verband tussen beide variabelen in alle onderzochte lammeren. Deze bevinding bevestigt de veronderstelling dat een verhoogde Pulsatility Index in de navelstrengslagader een teken is van een verminderde doorstroombaarheid van het foetoplacentaire bloedvaatstelsel (hoofdstuk 6).

Dateen afwijkende bloedstroomsnelheidscurve in een van beide navelstrengslagaderen nauwelijks betekenis hoeft te hebben voor de foetale gezondheidstoestand werd reeds gesuggereerd in de bestaande literatuur. Dat een afwijkende bloedstroomsnelheidscurve in een van beide navelstrengslagaderen ook nauwelijks betekenis hoeft te hebben voor de totale foetoplacentaire bloedstroom kon worden aangetoond door de snelheidscurven in de bloedstroom die de gehele placenta voorziet, te vergelijken met de snelheidscurven in de bloedstroom vlak bij een geëmboliseerd gedeelte van de placenta. De Pulsatility Index van de bloedstroom die de gehele placenta voorzag, toonde een consistente samenhang met de totale foetoplacentaire bloedstroom, terwijl de Pulsatility Index van de bloedstroom die slechts een gedeelte van de placenta voorzag, geen consistente samenhang vertoonde met de totale foetoplacentaire bloedstroom. Gesuggereerd wordt dat verloskundige besluitvorming op basis van een afwijkende bloedstroomsnelheidscurve in een van beide navelstrengslagaderen als onjuist beschouwd moet worden (hoofdstuk 7).

$\mathrm{Bij}$ de beoordeling van de foetale gezondheidstoestand of het effect van geneesmiddelen op de foetus, wordt een verandering in de foetale hartfrequentie veelal als enige criterium gehanteerd. Uit dierexperimentele studies blijkt echter dat in geval van stress, de foetale bloeddruk ook vaak aan verandering onderhevig is. Derhalve zou het zinvol kunnen zijn op indirecte wijze een indruk te krijgen aangaande de bloeddruk bij de humane foetus. Omdat bij stress-situaties een stijging van de systemische vaatweerstand en een stijging van bloeddruk hand in hand lijken te gaan, werd onderzoek gedaan naar de relatie tussen de Pulsatility Index en de bloeddruk in de grote lichaamsslagader, terwijl vaatvemauwende stoffen werden ingespoten (hoofdstuk 8). Onder deze omstandigheden, waarbij zich een forse bloeddrukverhoging ontwikkelde bij de foetus, was sprake van een zwak positief verband tussen beide variabelen. De zwakte van het verband wordt mogelijk verklaard door de instabiele omstandigheden waaronder de metingen verricht werden. Om op zinvolle wijze de relatie tussen de foetale bloeddruk en Doppler bloedstroomsnelheidscurven te onderzoeken, dient dit te gebeuren onder meer stabiele stress-situaties. 
Tenslotte wordt geconcludeerd dat dier-experimenteel onderzoek het inzicht in de diagnostische betekenis van foetale Doppler metingen kan doen toenemen. Wat de waarde is van Doppler metingen voor de verloskundige besluitvorming, kan alleen in klinische studies onderzocht worden. 


\section{Curriculum vitae}

The author of this thesis was born January 18, 1960, in Linne, The Netherlands. He attended secondary school (Gymnasium B) at the 'Bisschoppelijk College" in Roermond, where he graduated in 1978. In the same year, he went to Medical School at the Catholic University of Nijmegen and graduated in November 1985. In December 1985, he started as a resident at the Department of Obstetrics and Gynecology of the Canisius-Wilhelmina Hospital in Nijmegen (chairman: Dr W.B.K.M.V. de Goey). In May 1987, he became research fellow at the Department of Obstetrics and Gynecology of the State University Limburg (chairman: Prof. Dr J. de Haan). In July 1990, he started his specialist training in obstetrics and gynecology at the Department of Obstetrics and Gynecollogy of the Academic Hospital Maastricht (chairman: Prof. Dr J. de Haan). 